\title{
The complete European guidelines on phenylketonuria: diagnosis and treatment
}

\author{
A. M. J. van Wegberg ${ }^{1}$, A. MacDonald ${ }^{2}$, K. Ahring ${ }^{3}$, A. Bélanger-Quintana ${ }^{4}$, N. Blau ${ }^{5,6}$, A. M. Bosch7 , A. Burlina ${ }^{8}$, \\ J. Campistol ${ }^{9}$, F. Feillet ${ }^{10}$, M. Giżewska ${ }^{11}$, S. C. Huijbregts ${ }^{12}$, S. Kearney ${ }^{13}$, V. Leuzzi ${ }^{14}$, F. Maillot ${ }^{15}$, A. C. Muntau ${ }^{16}$, \\ M. van Rijn ${ }^{1}$, F. Trefz ${ }^{17}$, J. H. Walter ${ }^{18}$ and F. J. van Spronsen ${ }^{1 *}$
}

\begin{abstract}
Phenylketonuria (PKU) is an autosomal recessive inborn error of phenylalanine metabolism caused by deficiency in the enzyme phenylalanine hydroxylase that converts phenylalanine into tyrosine. If left untreated, PKU results in increased phenylalanine concentrations in blood and brain, which cause severe intellectual disability, epilepsy and behavioural problems. PKU management differs widely across Europe and therefore these guidelines have been developed aiming to optimize and standardize PKU care. Professionals from 10 different European countries developed the guidelines according to the AGREE (Appraisal of Guidelines for Research and Evaluation) method. Literature search, critical appraisal and evidence grading were conducted according to the SIGN (Scottish Intercollegiate Guidelines Network) method. The Delphimethod was used when there was no or little evidence available. External consultants reviewed the guidelines. Using these methods 70 statements were formulated based on the highest quality evidence available. The level of evidence of most recommendations is C or D. Although study designs and patient numbers are sub-optimal, many statements are convincing, important and relevant. In addition, knowledge gaps are identified which require further research in order to direct better care for the future.
\end{abstract}

Keywords: European, Guidelines, Phenylalanine hydroxylase deficiency, PAH deficiency, Phenylketonuria, PKU, Hyperphenylalaninemia, Phenylalanine, Treatment, Management, Recommendations,

Tetrahydrobiopterin, Sapropterin

\section{Background}

Phenylketonuria (PKU; McKusick \#261600) is a rare autosomal recessive inborn error of phenylalanine (Phe) metabolism caused by variants in the gene encoding phenylalanine hydroxylase (PAH). PAH normally converts Phe into tyrosine (Tyr) requiring the cofactor tetrahydrobiopterin (BH4), molecular oxygen and iron (Fig. 1) [1]. PAH deficiency leads to accumulation of Phe in the blood and brain. Untreated, PKU is characterized by irreversible intellectual disability, microcephaly, motor deficits, eczematous rash, autism, seizures, developmental problems, aberrant behaviour and psychiatric symptoms. The precise pathogenesis

\footnotetext{
* Correspondence: f.j.van.spronsen@umcg.nl

'Division of Metabolic Diseases, Beatrix Children's Hospital, University Medical Center Groningen, PO BOX 30.001, 9700 RB Groningen, The Netherlands Full list of author information is available at the end of the article
}

of brain dysfunction is still unclear (Fig. 2) [2]. As high blood Phe concentrations are strongly related to neurocognitive outcome, existing treatments aim at decreasing blood Phe concentrations. PKU was identified in 1934 by Følling when he detected phenylketone bodies in the urine of affected individuals and in 1953, Bickel first reported the effectiveness of a low-Phe diet in a child with PKU. In the 1960's, Guthrie developed a simple test to detect hyperphenylalaninemia (HPA) in large populations. This led to PKU becoming the first disorder to benefit from newborn screening; its early detection and treatment prevented mental retardation. However, the NBS screen is for HPA and this is defined as any blood Phe $>120 \mu \mathrm{mol} / \mathrm{L}$. Therefore, in every positive NBS for Phe, primary phenylalanine hydroxylase deficiency should be 


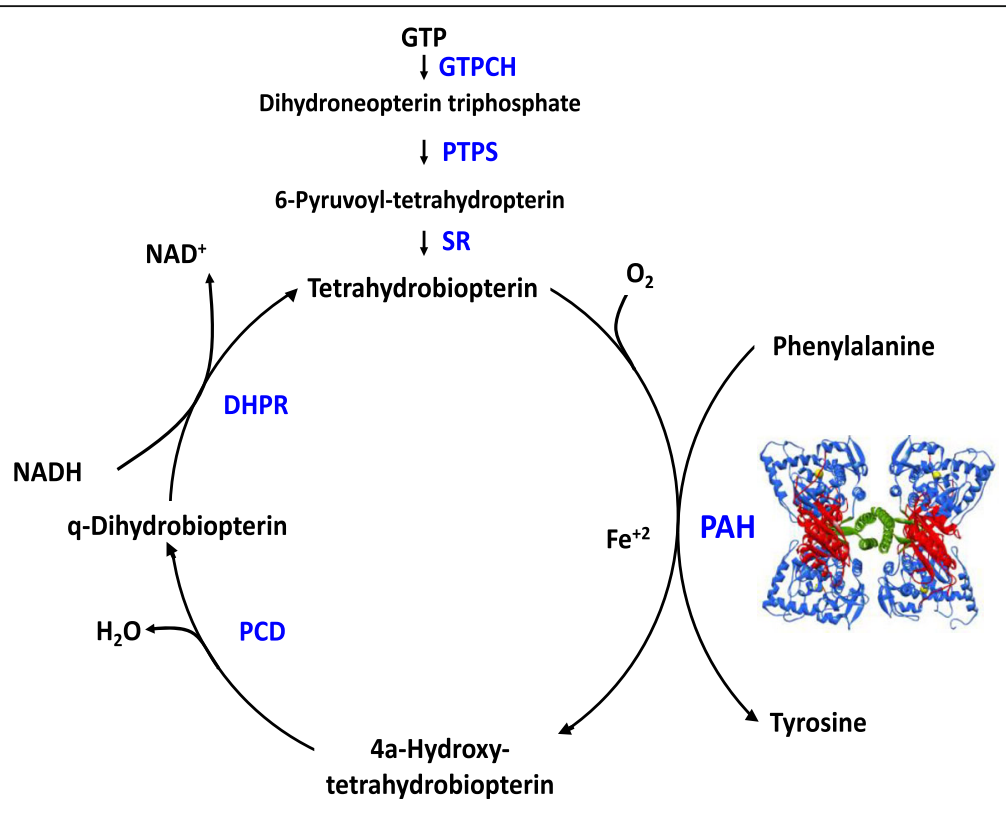

Fig. 1 Phenylalanine hydroxylating system. BH4: tetrahydrobiopterin; DHPR: dihydropteridine reductase; GTP: guanosine triphosphate; GTPCH: GTP cyclohydrolase I; Phe: Phenylalanine; PAH: phenylalanine hydroxylase; PCD: phenylalanine carbinolamie-4a-dehydratase; PTPS: 6-pyruvoyl-tetrahydropterin synthase; SR: sepiapterin reductase

distinghuished from other causes of HPA including pterin defects, high protein intake, liver disease or HPA not requiring treatment. This guideline is for PKU and does not discuss pterin defects which necessitate different treatment and follow-up [3].
The prevalence of PKU varies worldwide. In Europe, the mean prevalence is approximately 1:10,000 newborns with a higher rate in some countries such as Ireland and Turkey, and a very low rate in Finland [4].

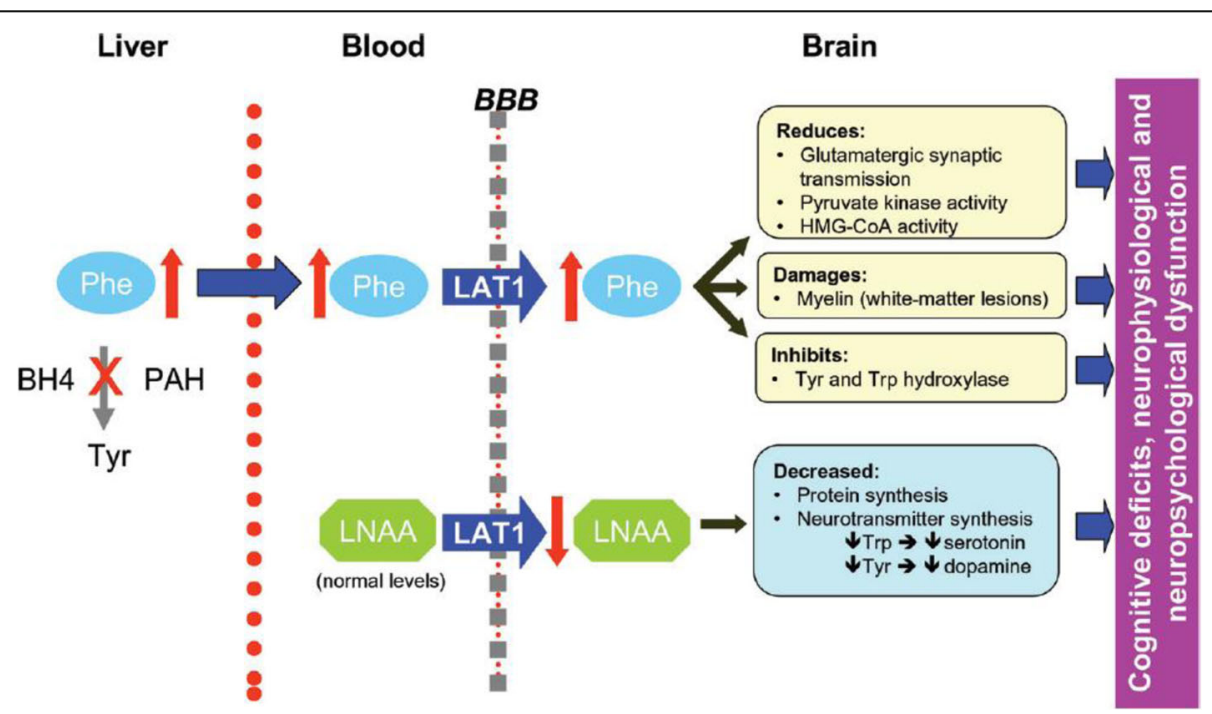

Fig. 2 Pathophysiology of PKU: Summary of potential mechanisms of neurocognitive impairment by high phenylalanine concentrations. Phe: phenylalanine; BBB, blood-brain barrier; LNAA: Large Neutral Amino Acids; LAT1, L-type amino acid carrier; BH4, tetrahydrobiopterin; HMG-CoA, 3-hydroxy-3-methylglutaryl-coenzyme A; Tyr, tyrosine; Trp, tryptophan 
Due to NBS and treatment commencement shortly after birth, patients fall within the broad normal range of general ability, attain more or less expected educational standards and lead independent lives as adults. As a consequence, PKU is considered a medical success story but neuropsychological deficits, behavioural and social issues occur in some patients, and (as a group) their mean neurocognitive always level is somewhat below their siblings or control groups from the general population $[1,5]$.

The cornerstone of PKU treatment is a low Phe diet in combination with Phe-free $\mathrm{L}$-amino acid supplements. Some PKU centres use casein glycomacropeptide (GMP) or large neutral amino acids (LNAA) as alternative dietary supplements. Certain patients are responsive to and are treated with $\mathrm{BH} 4$, acting as a pharmaceutical chaperone (prescribed as sapropterin dihydrochloride) [1]. Possible future treatments include enzyme substitution and gene therapy.

PKU management differs widely across Europe, even though the evidence on which management is based is the same [6-8]. Therefore, the development of European PKU guidelines was considered necessary [8-10] and initiated after the publication of the consensus paper by the European Society of Phenylketonuria and Allied Disorders (ESPKU) [11]. Guidelines can result in measurable improvements in patient care $[12,13]$, provision of consistent, highquality treatment without inequality, and rare disease awareness [14]. The key statements from this guideline were published recently [15]. The difficulty in rare disease guideline development is that high quality studies that include large patient numbers are scarse. Evidence is lacking in several areas including treatment initiation and adult management goals. Therefore, guidelines may change when new data is available. The goal of these European guidelines is to offer a standard for diagnostics, treatment and care in PKU that would lead to optimal clinical and neuropsychological outcome without overtreatment and unnecessary costs. These guidelines are intended to be used by metabolic physicians, dieticians, obstetricians, midwives, psychologists, social workers, biochemists and other professionals involved in the treatment of patients with PKU due to PAH deficiency.

\section{Methods}

The scientific advisory committee of the ESPKU was asked to invite a group of European PKU experts based on their expertise and experience rather than their nationality. Nineteen were invited; 1 declined and 1 resigned for personal reasons. The 17 remaining professionals were divided into 5 working groups and supported by a project lead (F.J. van Spronsen) and project assistant (A.M.J. van Wegberg). Working group members included 8 paediatric metabolic physicians, an adult metabolic physician, 2 paediatric neurologists, 1 biochemist, 3 metabolic dieticians and 2 (neuro) psychologists. Some assisted more than 1 working group and an obstetrician was consulted by the maternal PKU group. These guidelines were developed between October 2012 and December 2015.

The Appraisal of Guidelines for Research and Evaluation (AGREE) method was used to formulate the guidelines. The literature search, critical appraisal and evidence grading were performed according to the Scottish Intercollegiate Guidelines Network (SIGN) method version 2011 (http://www.sign.ac.uk/) (Table 1). There was one update (version 2014) as SIGN decided not to continue with the $\mathrm{ABCD}$ grading. At the start of these guidelines, development version 2011 was the appropriate methodology. Forthcoming updates will use the new GRADE process.

The 5 working groups defined key questions on the following 6 subjects: 1) Nutritional treatment and biochemical/nutritional follow up; 2) Neurocognitive outcome including imaging, psychosocial outcome and adherence; 3) Adult and maternal PKU; 4) Late diagnosed and untreated PKU; 5) Diagnosis of PKU including treatment initiation; and 6) Pharmacological treatment of PKU. They searched for relevant literature in PubMed (MEDLINE), EMBASE, NHS Economic Evaluations Database and The Cochrane Library being helped by the project assistant. For some subjects, additional search systems were used and reference lists were checked. All reviewed literature was published before Dec 31, 2015 and did not exclude any publications before a specified year or type of study design. Papers were excluded if they were not relevant to the key question or not written in English language. A total of 975 publications was reviewed. The methodological quality of the studies was assessed by 2 group members independently and/or by group discussion. Recommendations were either based on evidence (if level of evidence was A or B using the SIGN method) or by consensus using the Delphi method (if the level of evidence was $\mathrm{C}, \mathrm{D}$ or the so-called good practice points that are not based on any evidence). To reach such consensus, those recommendations without high level of evidence were discussed with all participants of all working groups during 5 face-to-face plenary sessions using Delphi methodology. All working groups and plenary sessions were facilitated by the guidelines lead and/or the project assistant. 
Table 1 SIGN grading system 1999-2012

\begin{tabular}{|c|c|}
\hline \multicolumn{2}{|c|}{ Levels of evidence } \\
\hline $1++$ & High quality meta-analyses, systematic reviews of RCTs, or RCTs with a very low risk of bias \\
\hline $1+$ & Well-conducted meta-analyses, systematic reviews, or RCTs with a low risk of bias \\
\hline $1-$ & Meta-analyses, systematic reviews, or RCTs with a high risk of bias \\
\hline $2++$ & $\begin{array}{l}\text { High quality systematic reviews of case control or cohort or studies } \\
\text { High quality case control or cohort studies with a very low risk of confounding or } \\
\text { bias and a high probability that the relationship is causal }\end{array}$ \\
\hline $2+$ & $\begin{array}{l}\text { Well-conducted case control or cohort studies with a low risk of confounding or } \\
\text { bias and a moderate probability that the relationship is causal }\end{array}$ \\
\hline $2-$ & $\begin{array}{l}\text { Case control or cohort studies with a high risk of confounding or bias and a significant } \\
\text { risk that the relationship is not causal }\end{array}$ \\
\hline 3 & Non-analytic studies, e.g. case reports, case series \\
\hline 4 & Expert opinion \\
\hline \multicolumn{2}{|c|}{ Grades of recommendations } \\
\hline A & $\begin{array}{l}\text { At least one meta-analysis, systematic review, or RCT rated as } 1++ \text {, and directly applicable } \\
\text { to the target population; or } \\
\text { A body of evidence consisting principally of studies rated as } 1+\text {, directly applicable to the } \\
\text { target population, and demonstrating overall consistency of results }\end{array}$ \\
\hline B & $\begin{array}{l}\text { A body of evidence including studies rated as } 2++ \text {, directly applicable to the target population, } \\
\text { and demonstrating overall consistency of results; or } \\
\text { Extrapolated evidence from studies rated as } 1++ \text { or } 1+\end{array}$ \\
\hline C & $\begin{array}{l}\text { A body of evidence including studies rated as } 2+\text {, directly applicable to the target population } \\
\text { and demonstrating overall consistency of results; or } \\
\text { Extrapolated evidence from studies rated as } 2++\end{array}$ \\
\hline D & $\begin{array}{l}\text { Evidence level } 3 \text { or } 4 \text {; or } \\
\text { Extrapolated evidence from studies rated as } 2+\end{array}$ \\
\hline \multicolumn{2}{|c|}{ Good practice points } \\
\hline$\checkmark$ & Recommended best practice based on the clinical experience of the guideline development group \\
\hline
\end{tabular}

Because of the rarity of this disorder, there were limited high quality papers available for most subjects, even though PKU is one of the most researched inherited metabolic disorders (IMD). Most papers described cohort/chart studies, crosssectional or descriptive studies, and therefore, most subjects and evidence did not exceed level C. Although the design of many studies was sub-optimal or they lacked statistical power, the statements written in this guideline are convincing, important and relevant.

Consistency, applicability and volume of evidence were considered with some evidence upgraded or downgraded accordingly. There was no grading system available for diagnostic accuracy evidence.

A concept of the guideline was sent to 16 external consultants specialized in PKU management. Fifteen of them responded, while 2 reviewers chose to remain anonymous; S. Beblo (Germany), G. Berry (US), M. Bik-Multanowski (Poland), M. Cleary (United Kingdom), T. Coşkun (Turkey), H. Gökmen-Özel (Turkey), J. Häberle (Switzerland), R. Lachmann (United Kingdom), H. Levy (United States), Y.
Okano (Japan), I. Schwartz (Brazil), J. Zeman (Czech Republic), and patient organization ESPKU.

For subjects where the evidence was unconvincing, this may be translated into daily practice as either: 1) no treatment/impact of guidelines until proven to be effective, or 2) treatment/implementation until proven otherwise.

A grant was received from the ESPKU to fund a project assistant. The ESPKU or other people outside the guideline team had no opportunity to influence the development of the guideline statements or the full guideline document (except the 14 professionals and the ESPKU when invited to provide their external review).

\section{Key recommendations}

The following recommendations were highlighted as the key clinical recommendations that should be prioritized for implementation [15]. The grade of recommendation relates to the scientific evidence and does not reflect the clinical importance. 


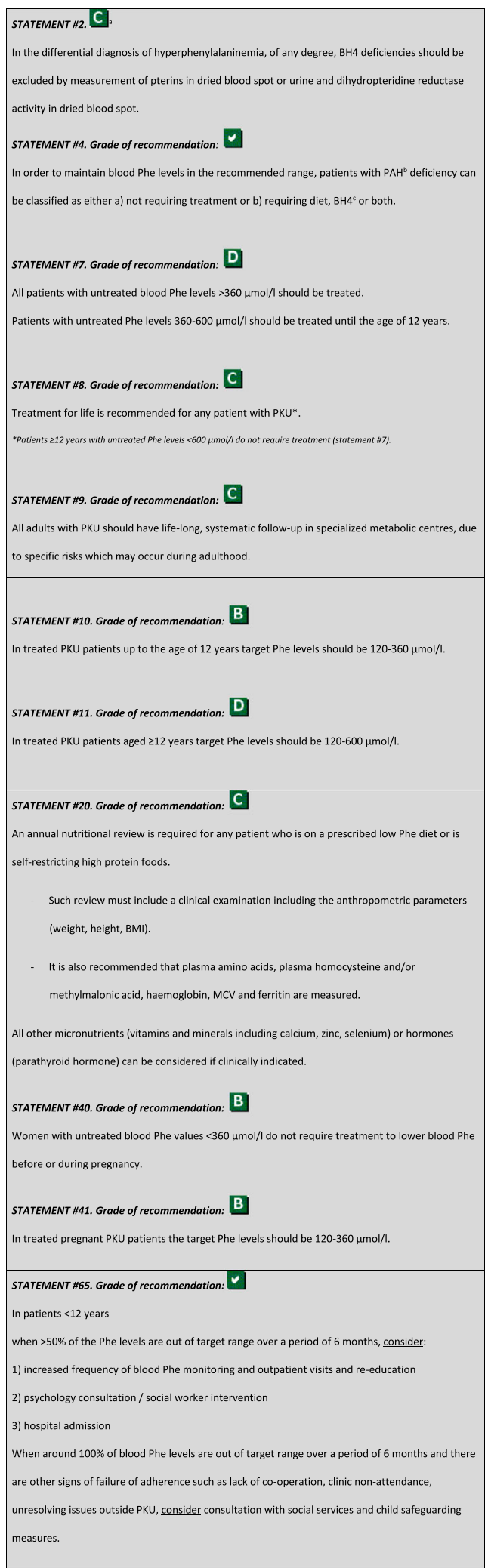

The marks range from $\mathrm{V}$ (no possibility to evaluate the level of evidence due to lack of any paper on this issue)

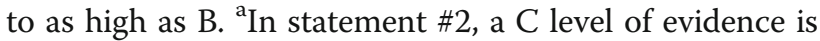
chosen because of the high number of data notwithstanding that most included papers are of descriptive nature; ${ }^{\mathrm{b}} \mathrm{PAH}$ : phenylalanine hydroxylase; ${ }^{\mathrm{C}} \mathrm{BH} 4$ : tetrahydrobiopterin; ${ }^{\mathrm{d}}$ Phe: phenylalanine.

\section{Diagnosis \\ Diagnosis}

Published evidence confirms that universal NBS for PKU meets all accepted screening criteria and justifies the cost and infrastructure necessary for the collection and testing of neonatal blood spots [16-18]. NBS is considered a national obligation even in countries when populations are known not to have PKU. Due to high migration in countries, a diagnosis of PKU remains possible. NBS requires: 1) a robust infrastructure in which blood is taken from all newborns (ideally between 24 and $72 \mathrm{~h}$ after birth (Collaborative Laboratory Integrated Reports at http:// clir.mayo.edu), to ensure timely start of treatment; and 2) a well-equipped laboratory that can handle bloodspots efficiently. Low-income countries may consider using the NBS laboratory facilities of other countries.

There are numerous committees and working groups that work on optimization of NBS procedures from the time of blood sampling, the method chosen for diagnosing high blood Phe levels and the referral procedure. At least partly, these procedures depend on national health care organizations. The most important issue is that children with a positive NBS result should be referred to a specialized metabolic centre with knowledge and experience in the diagnostic procedures and early treatment strategies to ensure the best outcome of PKU patients.

Individuals who have not had NBS and present with developmental delay or other PKU-related symptoms, should have plasma amino acids analysed.

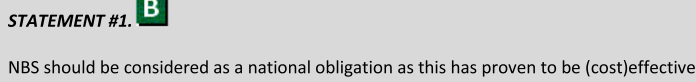

\section{Differential diagnosis of $\mathrm{BH} 4$ deficiencies}

The differential diagnosis of HPA includes high natural protein intake, prematurity, defects in $\mathrm{BH} 4$ metabolism and liver disease. Patients with disorders of $\mathrm{BH} 4$ metabolism including GTP cyclohydrolyase I (GTPCH) deficiency, 6-pyruvoyl-tetrahydropterin synthase (PTPS) deficiency, dihydropteridine reductase (DHPR) deficiency and pterin-4a-carbinolamine dehydratase (PCD) deficiency can present with any degree of HPA $[19,20]$. Some patients with GTPCH deficiency have normal Phe concentrations during the neonatal period $[20,21]$. 
Dopa responsive dystonia caused by the dominant form of GTPCH deficiency and sepiapterin reductase (SR) deficiency [22] are not associated with HPA. With the exception of DHPR deficiency, which can be detected by determination of DHPR activity in dried blood spots (DBS), all other forms of BH4 deficiency (GTPCH, PTPS, and PCD deficiency) can be detected by specific pterin patterns in urine or DBS $[19,23,24]$.

In cases where there may be delayed results of pterin and DPHR analysis, a 24-h BH4 loading test can be performed, in addition to analysis of pterins and DHPR that would allow earlier diagnosis of BH4-responsive PKU patients and/or BH4 deficiencies. Samples of blood and urine should be taken prior to starting treatment and before BH4 loading. Urine should be sampled and stored in dark conditions (by wrapping in aluminium foil) and stored immediately in a freezer. A useful alternative could be the use of next-generation sequencing panels $[25,26]$, but this methodology is only advisable when costs are lower and results are available within 7 days. Early diagnosis of GTPCH, PTPS and DHPR deficiencies may prevent irreversible brain damage by pharmacological treatment [20]. Those with PCD deficiency may be at risk of developing non-immune MODY-like diabetes or hypomagnesaemia and renal magnesium wasting $[27,28]$. Evaluation for BH4 disorders for any neonate or infant with neurological problems of unknown origin is suggested even without increased Phe or negative NBS for increased Phe.

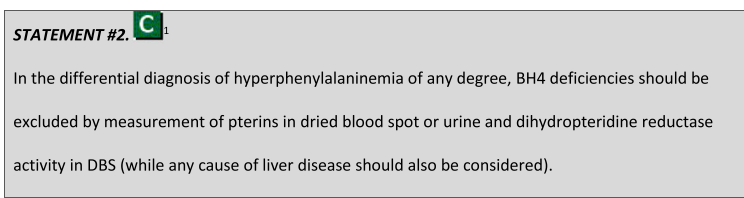

${ }^{1}$ Although most included papers are of descriptive nature the level of evidence is chosen to be $C$ because of the high number of data.

\section{Genotyping}

The gene encoding PAH is located on chromosome 12 (region q22-24.1) consisting of 13 exons and 12 introns, covering a total of $100 \mathrm{~kb}$ of genetic data. Over $950 \mathrm{PAH}$ variants ( $P A H v d b$ database; http://www.biopku.org/home/ pah.asp; last accessed 07-12-2015) are known to be associated with PAH deficiency. The majority of the variants $(60 \%)$ are missense, usually resulting in protein misfolding and/or impairment of catalytic functions.

Patient genotyping is not essential for the diagnosis of PKU but the genotype can determine the degree of protein dysfunction, residual PAH activity and consequently the metabolic phenotype. The classification of PAH genotypes may allow for prediction of the biochemical and metabolic phenotypes in many genotypes and be useful for the management of HPA in newborns [29-32]. Also, at least to some degree, $\mathrm{BH} 4$-responsiveness may be predicted or excluded from the patient's genotype [32-34]. Patients with gene variants that determine a high residual enzyme activity (which are those with the milder metabolic phenotypes) have a higher probability of responding to $\mathrm{BH} 4[35,36]$. Alleles that are known to be responsive to treatment with $\mathrm{BH} 4$ are listed in the BIOPKU database http://www.biopku.org/home/biopku.asp. Patients with a genotype known to be non-BH4-responsive should not undergo $\mathrm{BH} 4$ testing, while patients with a genotype with 2 BH4-responsive variations may directly proceed to a treatment trial rather than a $\mathrm{BH} 4$ loading test. In all other patients, a BH4 loading should be considered.

Prenatal diagnosis for PKU is feasible and genetic counselling depends on many issues including ethical, religious and legal issues in each country.

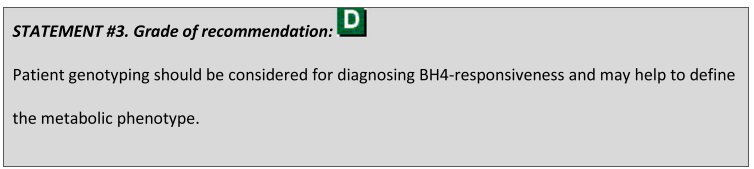

\section{PKU classification}

There is no consensus regarding phenotype classification. Blaskovics developed a Phe loading test to differentiate subtypes based on the responses among 8 HPA disease types of which 5 were related to PAH deficiency [37]. However, at present, this is not regarded as ethical as it increases the Phe level. In 1980, untreated Phe levels, e.g., those measured at clinical diagnosis, were used by Güttler for PKU phenotyping [38]. These criteria no longer aid in diagnosing patients for various reasons, including the large range of cut-off points [39] and even more importantly, the time of neonatal screening, as patients will commonly start treatment before reaching their maximal Phe concentrations [40]. Additionally, Phe tolerance is used to differentiate among 3 or 4 phenotypes [38, 41]. Exact Phe tolerance is difficult to determine because of non standardized conditions and discrepancies between prescribed and actual intake of Phe.Therefore, the following simplified classification scheme is suggested, derived from Blau [3].

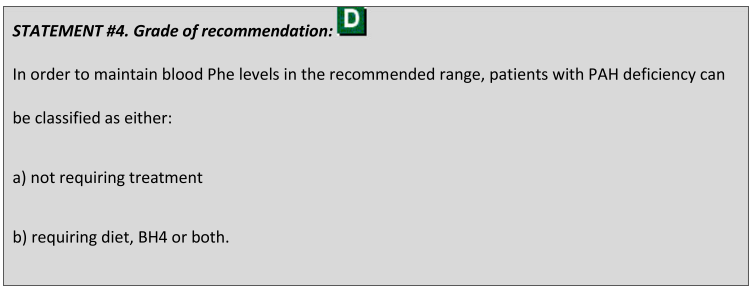




\section{Initiation of treatment and treatment for life Initiation of treatment}

In 1990, Smith et al. showed that every 4 weeks' delay in starting treatment caused a decline of IQ score by approximately 4 points [42], underscoring the knowledge that neurological damage starts early after birth. Although there are no formal studies to indicate that treatment commencement even earlier is necessary, data show that treatment in the early years of life has more impact than later years. As a consequence it is generally recommended that treatment should start as early as possible to prevent neurological damage [1]. We consider that treatment should be initiated before the age of 10 days, which for many countries will require change in timing of national NBS, logistical and diagnostic procedures.

There is unanimity in the literature and among professionals that patients with untreated blood Phe concentrations $>600 \mu \mathrm{mol} / \mathrm{l}$ should be treated.

Except for the publication by Gassio et al. [43], no study has investigated if patients with untreated blood Phe levels $<360 \mu \mathrm{mol} / \mathrm{l}$ should be treated. There is consensus that patients with untreated blood Phe levels $<360 \mu \mathrm{mol} / \mathrm{l}$ should remain untreated, as this is not considered to be indicative of disease. Gassio et al. [43] found that individuals with HPA but with Phe levels $<360 \mu \mathrm{mol} / \mathrm{l}$ without treatment, had scores on neuropsychological testing similar to control individuals except for 1 out of 2 executive function (EF) tests. However, this could also be explained by HPA patients having a lower average age than the control patients.

Because of the possibility of blood Phe concentrations increasing with age, patients with Phe levels $<360 \mu \mathrm{mol} / \mathrm{l}$ should be monitored (at a lower frequency) during the first year of life as a minimum $[44,45]$.

The evidence regarding initiation of treatment with blood Phe concentrations between 360 and $600 \mu \mathrm{mol} / \mathrm{l}$ is inconsistent. Campistol et al. [39] and van Spronsen [46] discussed this dilemma. Costello et al. [47] found a trend towards lower intelligence quotient (IQ) in those with higher Phe levels when comparing 3 groups $(<400$, $400-500$ and $>500 \mu \mathrm{mol} / \mathrm{l}$ ) and recommended treatment to maintain Phe $<400 \mu \mathrm{mol} / \mathrm{l}$ throughout childhood in all forms of PKU. It was predicted that for every $100 \mu \mathrm{mol} / \mathrm{L}$ increase in mean Phe that IQ would decrease by approximately 6 IQ points. However, the groups were very small ( $n=6, n=11$, and $n=7$ respectively) and the paper had some methodological weakness as the study included patients with untreated Phe concentrations $>600 \mu \mathrm{mol} / \mathrm{l}$. Diamond et al. [48] observed that 10 children with untreated Phe levels between 360 and $600 \mu \mathrm{mol} / \mathrm{l}$ did not perform as well as healthy control children, although this was not statistically significant. However, their mean Phe during the first month of life was $900 \mu \mathrm{mol} / \mathrm{l}$ which is also considered a methodological flaw. In 2001, Weglage et al. studied 31 patients with untreated blood Phe levels between 360 and $600 \mu \mathrm{mol} / \mathrm{l}$ [49]. This data showed normal neuropsychological outcome data, but only a small number of patients $(n=7)$ had untreated Phe levels in the higher range $(>500 \mu \mathrm{mol} / \mathrm{l})$ [49]. Smith et al. [50] also reported normal outcomes in 5 patients with untreated blood Phe levels between 360 and $600 \mu \mathrm{mol} / \mathrm{l}$ compared to matched controls. The number of patients having Phe levels just above 360 or just below $600 \mu \mathrm{mol} / \mathrm{l}$ was not reported. Because of limited data this publication was not considered [50]. An analytical shortcoming of previous studies is that patients were arbitrarily divided into subgroups. To examine the impact of Phe exposure in a vulnerable phase of brain development consider the use of more informative models like Widaman [51] did in maternal PKU. Therefore, we cannot give any definitive conclusions and consequently have decided to adopt a cautious approach. The evidence that supports treatment is of suboptimal quality. The evidence that supports no treatment is of better quality. However, the number of patients with blood Phe levels just below $600 \mu \mathrm{mol} / \mathrm{l}$ is considered too low and a different statistical analysis would be more informative. We recommend that patients with an untreated Phe concentration between 360 and $600 \mu \mathrm{mol} / \mathrm{l}$ should be treated during the first 12 years of age particularly as good metabolic control during childhood appears essential to prevent cognitive function impairment in PKU $[52,53]$.

For patients $\geq 12$ years old with untreated Phe levels $<600 \mu \mathrm{mol} / \mathrm{l}$ follow-up at a lower frequency is recommended, but remains particularly important in women due to the risks associated with maternal PKU when blood Phe levels are $>360 \mu \mathrm{mol} / \mathrm{l}$. Women need to be advised at each clinic that dietary treatment or BH4 therapy (or both) is essential pre-conception and during pregnancy. Some may consider that during child bearing years, women should continue a small dose of Phe-free L-amino acid supplements to help retain acceptance of its taste, but this practice remains unproven.

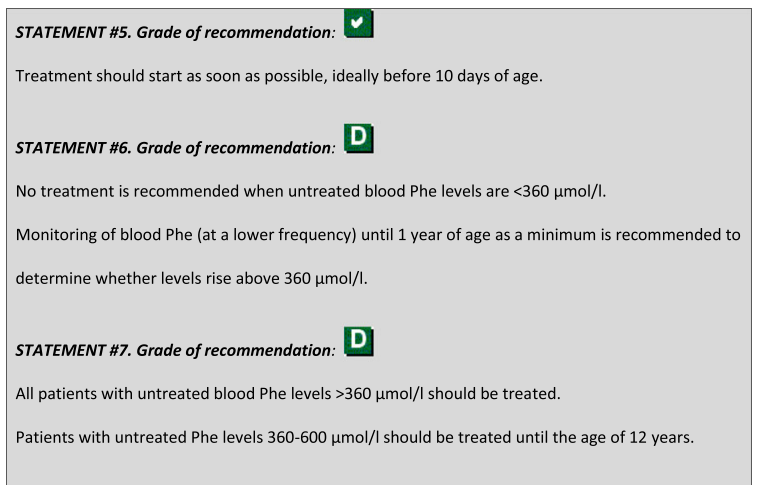




\section{Treatment for life}

Since the introduction of NBS and early treatment, patients with PKU no longer develop profound and irreversible intellectual disability. Over the last 40 years, studies have demonstrated that it is unsafe to stop treatment during childhood and pre-adolescence $[54,55]$. The foremost question now is if patients should be treated thoughout adulthood. There are no studies distinguishing the effect of Phe levels during different life phases (childhood, adolescence, adulthood). Also different terminology, target Phe levels and treatment strategies are given in published studies and consequently hamper a definitive conclusion. Here we describe studies in PKU patients who are continiously treated, on relaxed or discontinued diets and returned to diet.

Bosch et al. [56] reported that most early and continuously treated adults had a normal HRQoL even though dietary treatment is burdensome. Recently, a PKU related HRQoL questionnaire was developed, which assesses PKU-specific issues [57]. Bosch et al. [58] reported good HRQoL in 104 treated adult PKU patients with this PKU-specific and general questionnaire. Concerning neurological functioning, Fonnesbeck et al. [52] demonstrated an increased risk for low IQ with increasing Phe levels throughout life with a stronger association between blood Phe measured $<6$ years than later. In contrast, the meta-analysis of Albrecht et al. [59] indicated stable (but non-optimal) neurospychological speed test results with blood Phe levels between 750 and $1500 \mu \mathrm{mol} / \mathrm{l}$. However there were too little data to exclude the possibility that lower Phe levels could improve performance [59]. Over a 5 year period in adulthood, Weglage et al. [60] reported that the IQ, information processing and attention of 57 early treated PKU (ETPKU) adult patients remained constant, despite elevated blood Phe levels [60].

In patients on a relaxed diet, Bik et al. [61] reported that HRQoL was good in some of the adults, whereas others suffered from severe emotional stress. In a German study, Simon et al. [62] described that a lower number of patients with PKU had stable relationships and patients reached independency at a later age compared with the general population. It is unclear how these adults were treated, but probably dietary treatment was relaxed as this is the usual practice in Germany.

Adults with PKU who discontinued the low-Phe diet during adolescence have been reported to show significantly slower reaction times [63] and subtle differences in inhibition, attention and working memory [64] compared with adults on dietary restrictions and control groups. The older group (>32 y) of Weglage et al. [60] performed slower in terms of information processing, which might be related to their early relaxation of diet. Dietary discontinuation during adolescence was concluded by
Koch et al. [65] to be associated with poorer outcomes in adulthood regarding intellectual ability, achievement test scores and increased rates of medical and behavioural problems.

Some patients who experience suboptimal outcomes and return to diet improve. In adults, the reported neurological complications $(n=4)$ [66] and vision loss $(n=2)[67,68]$ all improved or even reversed when Pherestricted diet with Phe-free L-amino acid supplements was reinstituted [66-68]. In addition Schmidt et al. [69] reported reversible effects on sustained attention and calculation speed in a trial with 15 adults. Ten Hoedt et al. [70] showed in a randomized double-blind cross-over design study that short-term high Phe levels had a significant direct negative effect on mood and sustained attention in 9 adults. Returning to dietary restrictions has been shown to improve HRQoL in many of the adults with PKU who have been studied $[61,71]$. However, it is possible that adults who have no desire to return to diet may not participate in studies.

Overall it is unclear how many adults experience suboptimal outcomes that have impact on daily functioning. It is also not fully understood which consequences during adulthood are due to Phe levels before adulthood and/or during adulthood, and which of these consequences is improved by decreasing blood Phe during adulthood. Neither, it is clear if Phe levels during adulthood will impact outcome in elderly patients.

As there is currently no strong evidence that it is safe to discontinue dietary treatment in adults, treatment for life is recommended, even though it is acknowledged that dietary management is associated with significant patient burden. Returning to the diet is very challenging if patients have eaten high protein foods and/or find the Phe-free-L-amino acid supplements distasteful. Patient motivation should be strong with a supportive family network and metabolic team to overcome any barriers.

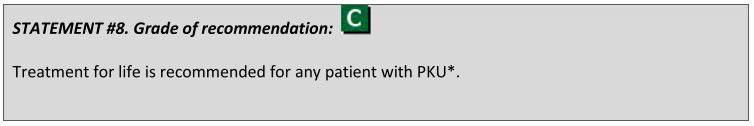

*Patients $\geq 12$ years with untreated Phe levels <600 $\mu \mathrm{mol} / \mathrm{l}$ do not require treatment (statement \#7).

\section{Life-long follow up}

Evidence from a systematic review demonstrates that significant sub-optimal outcomes exist in ETPKU adults. Issues include EF deficits, attention problems, decreased verbal memory, expressive naming and verbal fluency, as well as social and emotional difficulties [5]. ETPKU adults usually show a clear relationship between concurrent blood Phe concentrations and certain aspects of brain function, brain metabolism and differences in myelination 
as summarized by van Spronsen et al. [72]. Some adults who have not been treated early and continuously have been reported to develop neurological complications such as leukoencephalopathy, spastic paraparesis, brisk reflexes, tremor, Parkinsonism, psychiatric symptoms $(n=4)$ [66] and vision loss $(n=2)[67,68]$. Tremors have also been detected in ETPKU, although they are more frequent and severe in late treated patients [73]. At present, it is not known how many patients have neurological and psychological problems and which adult PKU patients have a higher risk of these problems. Many adults with PKU have a vegan-like diet but may not take Phe-free L-amino acid supplements [74] and consequently may be at risk of micronutrient deficiencies [75]. There is increasing reports of females (and not males) with PKU being overweight and obese [76, 77]. The risk of comorbidities makes dietary management more complex [78]. The risk of low bone density has widely been acknowledged but the risk of bone fractures is still unclear [79].

In PKU, life-long, systematic follow-up is recommended independent of the degree of adherence and (non-) treatment choice, to screen for long-term complications at any life stage, and provide appropriate support to patients. In addition, it is not known if there will be further complications when adult PKU patients advance in age, such as neurodegeneration or movement problems. By collecting data, we should be able to identify if patients are likely to deteriorate and which patients are at special risk of deterioration and why.

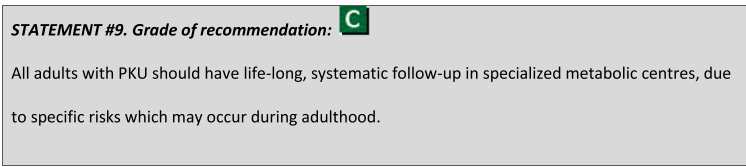

\section{Treatment goals and follow-up}

The primary goal of treatment is normal neurocognitive and psychosocial functioning. Blood Phe concentrations remain the best surrogate measure, and should be monitored regularly, aiming for blood Phe levels that stay within a given target treatment range, defined for a given age. Discussions on target ranges have focused primarily on the upper blood Phe level but there is little data to support the lower target level. The widely used lower target level of $120 \mu \mathrm{mol} / \mathrm{l}$ is derived from published cases describing adverse consequences at very low Phe levels $[80,81]$, and from past knowledge that the primary use of the Guthrie test was not sensitive in detecting lower Phe levels. It is now well established that blood Phe decreases during the day with the highest blood Phe attained early in the morning, following an overnight fast [82]. We advise a lower target level at $120 \mu \mathrm{mol} / \mathrm{l}$ until more data is available.
When trying to reach consensus about the upper target Phe concentration for treatment in PKU, comparison of studies was hampered by various factors:

- Studies report blood Phe in different ways (e.g. concurrent, lifetime as a mean, lifetime as median, or lifetime means of medians). Studies use different methods to measure blood Phe (past data were sometimes based on the semi-quantitative Guthrie or more reliable fluorometric enzyme analysis but more recently amino acid concentrations were usually measured by highperformance liquid chromatography and tandem mass spectrometry that are more precise). Differences between the methods (except for Guthrie method) are relatively small [83-85].

- Studies use different Phe samples such as venous serum, venous plasma and DBS. Past studies are largely based on plasma Phe levels, where it is now routine practice to perform DBS measurements. Differences between venous serum and venous plasma are usually regarded as minimal with a variation of $1 \%$ [86], but differences between DBS and plasma may be greater with DBS being reported to be 8-26\% lower [84, 86, 87]. It should be considered that a higher plasma Phe is likely to result in a higher variation between DBS and plasma. - There can be variations in Phe results due to variety in measurement in the DBS itself, haematocrit, the volume taken from the DBS, and the punch location [87-90]. At the same time, it is also reported that reliable Phe levels can be estimated within a minimum size of blood spot [91].

- Studies do not consistently include confounding factors such as maternal education, socioeconomic status and age at start of treatment.

The statements in this guideline recommend blood Phe as upper target levels where reported studies used means or mean of medians. Therefore, these upper target levels are probably on the safe side (considering current evidence), so even with differences in blood levels due to sample type, we still consider we have a reasonable upper target for Phe levels.

\section{Target Phe levels for children and adolescents}

Albrecht et al. [59] performed a meta-analysis including 20 studies focusing on neuropsychological speed tests of 7 different categories. In total, 509 patients (229 children, 106 adolescents and 174 adults) and 433 controls participated in these studies. The meta-analysis predicted no differences with controls when concurrent Phe concentrations reached $320 \mu \mathrm{mol} / \mathrm{L}$ for children between 7 and 13 years and up to $570 \mu \mathrm{mol} / \mathrm{L}$ for adolescents between 13 and 18 years of age [59]. Waisbren et al. [53] performed a meta-analysis examining the correlation 
between IQ and Phe levels reported in 40 different publications. They concluded that a difference in Phe level of $100 \mu \mathrm{mol} / \mathrm{l}$ between birth to $6-12$ years predicted a difference in IQ between 1.3 to 3.1 points in patients whose Phe levels ranged from 423 to $750 \mu \mathrm{mol} / \mathrm{l}$. With lifetime Phe levels, an increase of $100 \mu \mathrm{mol} / \mathrm{l}$ predicted an average 1.9 to 4.1 point reduction in IQ over a range of Phe from 394 to $666 \mu \mathrm{mol} / \mathrm{l}$ [53]. For example, someone with a Phe level of $500 \mu \mathrm{mol} / \mathrm{l}$, on average had a 1.9 to 4.1-point lower score on an IQ-test compared to someone with a Phe-level of $400 \mu \mathrm{mol} / \mathrm{l}$. Fonnesbeck et al. [52] performed a meta-analysis of 17 studies (432 individuals with PKU, aged 2-32 years) and addressed the relationship between the probability of an IQ less than 85 and Phe levels. Both life time Phe levels (more than 12 months before IQ measurement) and concurrent Phe levels (within 6 weeks of IQ-measurement) were considered [52]. The healthy population probability of an IQ less than 85 was approximately 15\%. For PKU patients the probability was $14 \%$ when the mean Phe level during the time frame of $\geq 6$ years of age was $400 \mu \mathrm{mol} / \mathrm{l}$ but increased to $20 \%$ when the mean Phe level was $600 \mu \mathrm{mol} / \mathrm{l}$. Before $<6$ years of age the probability was already $19 \%$ when the mean Phe level was $400 \mu \mathrm{mol} / \mathrm{l}$ and increased to $30 \%$ when the mean Phe level was $600 \mu \mathrm{mol} / \mathrm{l}$. A stronger association was observed between Phe levels during early childhood and later IQ. There was no strong association between concurrent Phe levels and IQ [52]. Taken together, in childhood, the meta-analyses of Albrecht et al. [59] and Waisbren et al. [53] suggests an upper target Phe concentration of 320 (age 7-13 years), and $423 \mu \mathrm{mol} / \mathrm{L}$ (birth to $6 / 12$ years), while the metaanalysis of Fonnesbeck et al. [52] suggested that a mean of $400 \mu \mathrm{mol} / \mathrm{L}$ ( $<6$ years) is already too high as it was associated with an increased risk of an IQ $<85$. It should be noted that the primary papers considered in these meta-analyses are mostly non-experimental designs such as (historical) cohorts, cross-sectional designs and case series, which in turn decreased the quality of these analyses.

Diamond et al. [48] showed in 37 PKU patients aged 6 months to 7 years that those with concurrent Phe levels (mean Phe from a 6 week period preceding testing) of $360-600 \mu \mathrm{mol} / \mathrm{l}$ performed less well in EF tasks requiring working memory and inhibitory abilities than did children with concurrent Phe levels $<360 \mu \mathrm{mol}$ and controls. In addition, PKU children with concurrent Phe levels $360-600 \mu \mathrm{mol} / \mathrm{l}$ had significantly lower IQ scores than did control subjects, although all participants scored within the normal range [48]. In a study by Leuzzi et al. [92], 9 PKU patients with Phe levels $>400 \mu \mathrm{mol} / \mathrm{l}$ performed worse than $5 \mathrm{PKU}$ patients with levels $<400 \mu \mathrm{mol} / \mathrm{l}$ and IQ- and age-matched controls (8-13 years) in all 7 tests, although not all differences were significant. PKU patients with Phe levels $<400 \mu \mathrm{mol} / \mathrm{l}$ performed comparably with controls in all tests but the Elithorn's Perceptual Maze Test [92]. In addition, Huijbregts et al. [93] found that 38 PKU patients with concurrent Phe $>360 \mu \mathrm{mol} / \mathrm{l}$ performed significantly worse in several tests targeting EF than matched controls. Patients with concurrent Phe levels $<360 \mu \mathrm{mol} / \mathrm{l}(n=29)$ did not differ from controls and performed significantly better than patients with concurrent Phe levels $>360 \mu \mathrm{mol} / \mathrm{l}$ [93].

Schmidt et al. [69] (included in the meta-analysis of Albrecht et al. [59]) reported 4 groups of PKU patients (mean age 9 years). Group A had good metabolic control (from birth to the age of 9 years) and had a concurrent Phe level of $240 \mu \mathrm{mol} / \mathrm{l}(n=31)$. Group B had good metabolic control up to the age of 9 years, but had a concurrent Phe level of $620 \mu \mathrm{mol} / \mathrm{l}(n=30)$. Group C and $\mathrm{D}$ were not in good metabolic control and had a concurrent Phe level of $520 \mu \mathrm{mol} / \mathrm{l}$ and $970 \mu \mathrm{mol} / \mathrm{l}$ $(n=32)$. Group A performed as well as the control group and better than group B, C and D for sustained attention and calculation speed tests. All the other groups performed worse than the control group [69].

Jahja et al. [94] examined inhibitory control, cognitive flexibility and motor control in 3 groups of PKU patients (aged 6-15) with different lifetime Phe levels and healthy controls $(n=73)$. The 3 groups had lifetime Phe levels of $\leq 240 \mu \mathrm{mol} / \mathrm{L}(n=10)$, between 240 and $360 \mu \mathrm{mol} / \mathrm{L}$ $(n=33)$ and $\geq 360 \mu \mathrm{mol} / \mathrm{l}(n=21)$. The patients with Phe levels below $\leq 240 \mu \mathrm{mol} / \mathrm{l}$ performed better than the other 2 PKU groups and equally well as the control group [94]. However, despite statistical significant differences, this was not considered clinically significant.

Moyle et al. [95] performed a meta-analysis of neuropsychological testing. PKU literature often combines data from children, adolescents and adults but this compromises the ability to interpret the results. Moyle included 11 papers focusing on adolescents (13-18 years) and adults ( $>18$ years). The level of dietary adherence was not uniform, although the majority of patients was following a relaxed diet at the time of testing. Additionally, the matching criteria and type of control groups differed across studies. The results from the study indicated that continuously treated PKU patients (without correcting for treatment adherence), while displaying no significant weakness in working memory, are likely to show reduced levels of functioning across a range of different cognitive functions (IQ, attention, inhibition, processing speed, and motor control) compared to controls [95].

Weglage et al. [60] examined adults with early-treated classical PKU to assess neurological and neuropsychological performance. At baseline, 28 patients were aged $<32$ years and 29 were $>32$ years. The older group relaxed the diet at the age of 10 years, while the younger group relaxed the diet in early adulthood. Significant differences 
were observed in Phe levels between the ages of 11 and 16 years. When studied for a 5 year period in adulthood, both groups remained constant in their performance. The older group, however, performed more slowly in testing for information processing, which might be related to their early relaxation of diet. From the age of 11 until 16 years, in the younger age group the median annual Phe varied between 496 and $707 \mu \mathrm{mol} / \mathrm{l}$ and for the older group, between 750 and $1038 \mu \mathrm{mol} / \mathrm{l}$ [60]. In summary, for adolescents the meta-analysis of Albrecht et al. [59] recommended a target Phe level of $570 \mu \mathrm{mol} / \mathrm{l}$ (age 1318 years), whereas the findings of Weglage et al. [60] suggest an upper target level between 496 and $707 \mu \mathrm{mol} / 1$ (age 11-16 years). The meta-analysis of Fonnesbeck et al. [52] and Waisbren et al. [53] are more difficult to interpret as they refer to lifetime Phe levels.

The evidence for patients $<12$ years of age is strong indicating that a Phe concentration of $360 \mu \mathrm{mol} / \mathrm{l}$ should be considered as the upper target Phe concentration. It could be argued that within this age group the upper target Phe levels needs to be lower (Schmidt et al. [69], Jahja et al. [94]), but at present time the evidence to lower the upper target Phe is not robust enough. If possible, meta-analysis of the data available studying the relationship between neurocognitive and neuropsychological outcome and blood Phe concentrations, examining if upper Phe levels other than $360 \mu \mathrm{mol} / \mathrm{l}$ give even better results are necessary, stressing the need for collaboration on an international level [51].

The evidence for patients $>12$ years of age is mainly indirect, as there are no studies investigating the effect of Phe levels during adolescence in patients who were in good metabolic control during childhood. Taking into acount the lower grade of evidence, an upper target Phe level at $600 \mu \mathrm{mol} / \mathrm{l}$ between ages 12 and 18 years is recommended.

\section{Target Phe levels during adulthood}

In adulthood the goal of treatment is to achieve normal neurocognitive and psychosocial functioning. As previously discussed, it is not fully understood which PKU adult outcomes are associated with increased Phe levels during adulthood and there are no large controlled longitudinal studies to help determine the optimal upper target blood Phe levels. Further data collection by long-term international collaborative studies is required to help direct current recommendations.

In the double-blind randomised placebo-controlled cross-over trial of Ten Hoedt et al. [70], 9 patients received Phe-loading and placebo-Phe-loading. Mean plasma Phe concentrations were $1259 \mu \mathrm{mol} / \mathrm{L}( \pm 332 \mu \mathrm{mol} / \mathrm{l})$ versus $709 \mu \mathrm{mol} / \mathrm{l}( \pm 322 \mu \mathrm{mol} / \mathrm{l})$, respectively. The higher Phe levels significantly worsened mood and sustained attention [70]. In Schmidt's et al. (1996) controlled experimental study, 15 early treated adults with normal IQ were tested 3 times; with their usual diet, a Phe-restricted diet and again their usual diet. Mean Phe levels were $1320 \mu \mathrm{mol} / \mathrm{l}$ (720-1800 $\mu \mathrm{mol} / \mathrm{l}), 630 \mu \mathrm{mol} / \mathrm{l}(280-966 \mu \mathrm{mol} / \mathrm{l})$ and $1410 \mu \mathrm{mol} / \mathrm{l}(1040-2200 \mu \mathrm{mol} / \mathrm{l})$, respectively. Sustained attention and calculation speed improved significantly with the lower Phe levels [69].

Channon et al. [64] compared 25 treated adults on diet with 25 adults who stopped treatment from 10 years of age onwards. The treated adult patients had a better performance for IQ, n-back accuracy and flanker speed, although the Phe levels differed significantly from 5 years of age onwards between the 2 groups. The range of mean 4yearly Phe levels was $460-870 \mu \mathrm{mol} / \mathrm{l}$ for the adults who remained on treatment, and $560-1410 \mu \mathrm{mol} / \mathrm{l}$ for the offdiet group. The on-diet adults performed worse compared to controls regarding n-back speed [64]. With these studies, it is difficult to interpret if consequences are due to Phe levels during childhood, adolescence or adulthood. Adulthood enables more invasive techniques to be used to determine safe Phe concentrations. Hoeksma et al. [96] using positron emission tomography, showed that plasma Phe concentrations $>600-800 \mu \mathrm{mol} / \mathrm{l}$ decreased cerebral protein synthesis rates in adults $(n=16)$ [96]. In several studies in PKU, but mainly with adolescents and adults, no white matter alteration (WMA) is observed when blood Phe is $<300 \mu \mathrm{mol} / \mathrm{l}$ or in some cases $<600 \mu \mathrm{mol} / \mathrm{l}$ [49, 97-100]. Blood Phe control and its impact on oxidative stress has also been considered. Oxidative stress occurs in neurodegenerative disease and the brain has relatively low levels of antioxidant defences. Sanayama et al. [101] reported oxidative stress changed greatly at a blood Phe level of $700-800 \mu \mathrm{mol} / \mathrm{l}(n=40)$ and thereby recommended Phe levels $<700-800 \mu \mathrm{mol} / \mathrm{l}$ [101].

The evidence, as strong or weak as it is, indicates $600 \mu \mathrm{mol} / \mathrm{l}$ as the upper target level, while no study could be found to support an upper target blood Phe level of $360 \mu \mathrm{mol} /$ [102]. It is recognized that an upper target Phe level of $600 \mu \mathrm{mol} / \mathrm{L}$ increases the dietary burden of care and may provide more challenges for patients returning to dietary treatment but this was not a determining factor in recommending this upper target Phe level.

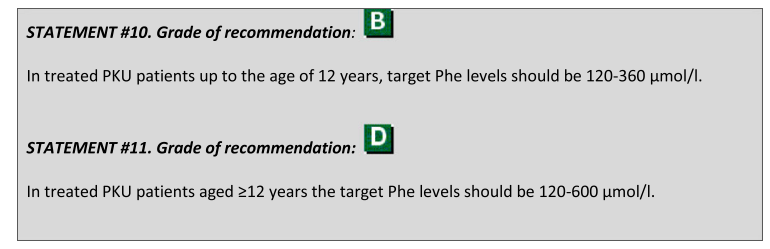

(See subparagraph maternal PKU for recommendations regarding maternal $\mathrm{PKU}$ )

Biochemical marker used for assessment of metabolic control Blood Phe levels (but not Phe fluctuations and Phe: Tyr ratios) are the primary reported markers of metabolic 
control [52, 53, 59]. Treatment is adjusted according to the blood Phe level. The effect of a single Phe levels outside the target range is not easily measured. Phe fluctuations over $24 \mathrm{~h}$ appear to be more related to uneven administration of Phe-free L-amino acid supplements [103], rather than the fasting/postprandial state or uneven distribution of natural protein allowance $[82,104]$.

There are data indicating that fluctuations in Phe (often measured as SD or SEE) can be a predictor of IQ [105-107], EF [106] and motor control [94], although the literature is inconsistent [108]. As Cleary et al. [109] described, it is difficult to distinguish the effect of more severe PKU and/or poor metabolic control from the effects of Phe fluctuations. Additionally, further research is needed to examine the differences between the shortterm and long-term effect of Phe fluctuations [109].

Considering the Phe: Tyr ratio, it is hypothesed that an increased Phe: Tyr ratio leads to dopamine deficiency as Phe and Tyr compete to cross the blood-brain barrier [48]. Jahja et al. [94] concluded, using multiple regression analysis $(n=64)$, that increased Phe: Tyr ratios were associated with poorer inhibition control [94]. Sharman et al. associated Phe: Tyr ratios with EF (T-scores from Behaviour Rating Inventory of Executive Function) in 2 papers partially using the same subject sample $(n=11$ and $n=12$ ). They suggested that a lifetime Phe: Tyr ratio of $<6$ was associated with a normal EF outcome, but this requires further evaluation by others $[110,111]$. Furthermore, in 2012, Sharman et al. found significant correlations between depressive symptoms and long-term exposure to either a high Phe:Tyr ratio or low Tyr, although the 18 adolescents with PKU scored within the normal range for depressive symptoms [112]. Luciana et al. [113] reported an association of the Phe: Tyr ratio with several aspects of cognitive functioning in a group of 18 PKU patients. Again, it was difficult to distinguish between the effect of Phe: Tyr ratio and the elevated Phe levels. Probably, the Phe: Tyr ratio is useful, but as the Tyr concentration depends on the timing of blood sampling $[82,114]$, the marker is only of value if measured after an overnight fast. Therefore, the exact value of the Phe: Tyr ratio in addition to blood Phe measurements remains to be determined.

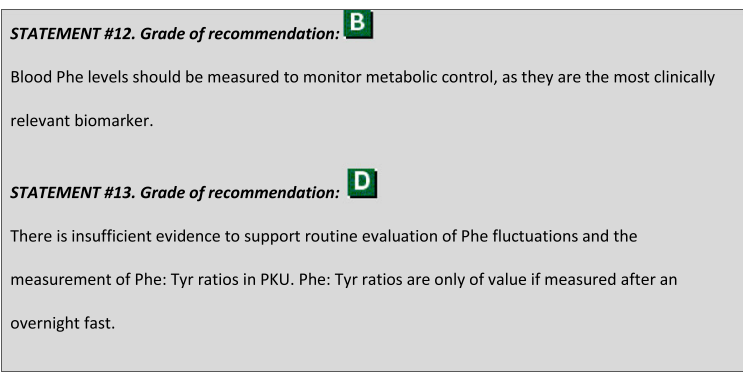

\section{Frequency of blood Phe measurements and outpatient visits}

Patients are monitored with home blood sampling and outpatient visits. The effect of frequency of contact or regularity of blood sampling on adherence has not been adequately assessed in PKU. Frequent contact during the first year of life is essential to instruct parents and help attain good metabolic control. Regular follow-up during adolescence is also crucial as it is well established that blood Phe control deteriorates [115]. After the age of 12 years, patients with PKU should aim for blood Phe levels of 120 $600 \mu \mathrm{mol} / \mathrm{L}$. It is essential that adolescents are supported throughout the transition process until they are established and confident in an adult care environment; they should be encouraged to take responsibility for self care, taking regular blood Phe samples, attending age appropriate outpatient clinics with suitable education programmes.

We suggested the following minimum frequencies of blood sampling and minimum outpatient visits for each age group:

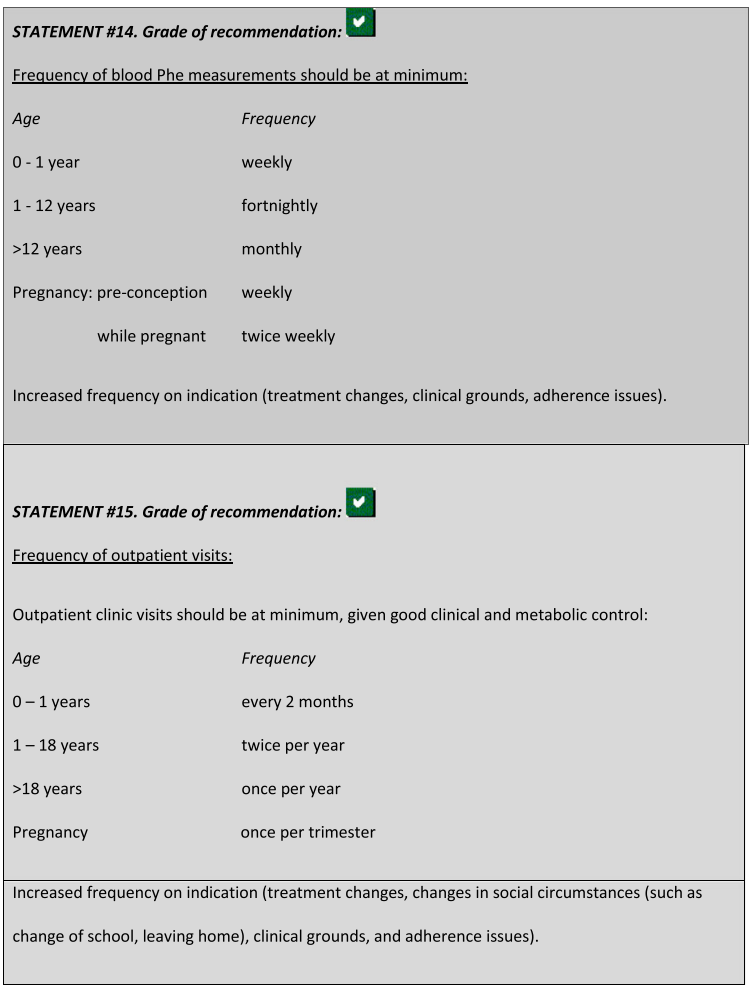

During the first year of life and throughout preconception and pregnancy, weekly (telephone) contact with health professionals is important to provide close support to patients and their families. Various life events, such as change of school, starting employment, living independently, as well as adherence issues (e.g. during adolescence) may necessitate a higher frequency of blood Phe testing and/or visits. 
It is important that blood Phe samples should be obtained at the same time of the day. To estimate the highest Phe value of the day and reliable Tyr levels, blood samples should be collected in the morning after fasting overnight. Blood Tyr levels taken at different times may be increased by the tyrosine intake from Phe-free Lamino acid supplements.

The time between bloods sampling and patients/parents receiving the results should be minimized, aiming for less than 5 days. In special situations such as infancy and maternal PKU, results should be available within 2-3 days of blood sampling. This requires home monitoring systems instead of home sampling.

At each outpatient visit, the following should be conducted: a medical and dietary history, assessment of anthropometry including body mass index estimation, and a physical and neurological examination, especially observing for clinical signs of Phe toxicity and nutrient (including Phe) deficiency $[80,81]$. Clinic reviews should always include a discussion on treatment issues and mental and physical health (e.g. neurological and psychiatric issues, behaviour and mood). Any additional investigations necessary are outlined in Table 2.

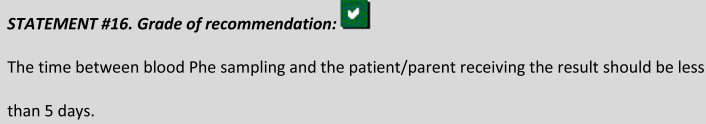

\section{Metabolic team and transition}

All patients should be treated in a specialized metabolic centre with a specialized metabolic laboratory. The minimum health professionals within a team for patients of all ages should be a metabolic physician and a dietician with experience in IMD. Access to a psychologist is requested by the ESPKU patient organization [11] while we strongly advise access to a (neuro)psychologist and social worker. It is recognized that PKU is a IMD possibly necessitating may necessitate the support of professionals outside the core team. That support can be for financial issues and beyond. Although in many countries adult patients are followed up by a paediatric team [116], it is important that metabolic teams prioritise the establishment of an adult metabolic service, lead by an adult metabolic physician, specifically trained in the management of IMD.

The process of transferring children to adult care should be conducted under a carefully structured 'transitional' process, beginning from around the age of 12 years. During this time, management should change from being parent/caregiver directed to patient controlled. This latter process must occur even if the patient is staying under the same paediatric service. Patients and families need an individualized care plan and timetable for transition, together with detailed information about the adult centre. This should be jointly written with teenagers, caregivers, and health professionals. This plan should include treatment goals, a timetable for transfer, and ensure there is a consistent approach between all health professionals. It should also provide a mutual understanding of the transition process. It has been demonstrated in PKU, with careful planning, close liaison between paediatric and adult teams, and patient and caregiver involvement, that most patients are able to make a successful transition to adult care [117]. There is no right time or age for the subsequent transfer of patient care to the adult treatment centre to occur but is commonly between 16 to 18 years of age, although some flexibility may be required depending on the maturity and circumstances of the patient.

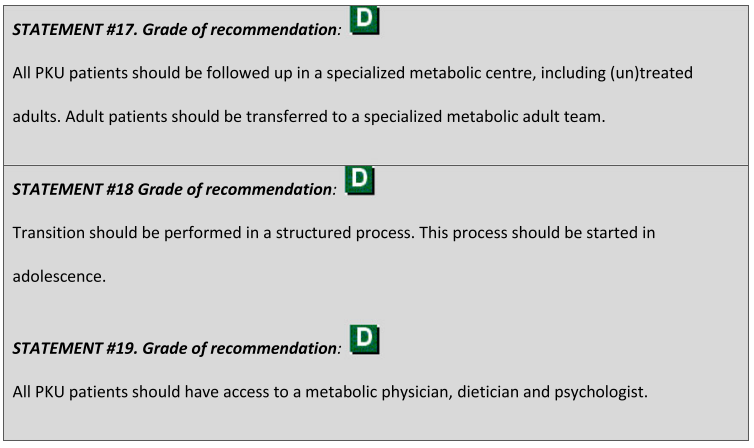

\section{Nutritional follow-up}

The nutritional status of patients varies according to PKU severity and type of treatment. Except for patients on a normal diet (MHP and fully BH4-responsive patients), the majority follow a low natural protein diet with limited or no animal protein sources. The major source of micronutrients is from supplemented Phe-free L-amino acids and if the intake of Phe-free L-amino acid supplements is suboptimal, this will increase the risk of micronutrient deficiency (e.g. iron, zinc, selenium and vitamin B12) [118-120].

Clinical symptoms of nutrient deficiency are rarely reported, and are mainly described for vitamin B12 deficiency in patients who have reduced or stopped their micronutrient supplement or Phe-free L-amino acid supplements while following a vegan-style diet [121, 122]. For some nutrients, the bioavailability appears sub-optimal (e.g. zinc [118, 123, 124] and iron [118, 125-127]).

Functional markers of micronutrient status (ferritin, hemoglobin, MCV for iron; methylmalonic acid and total homocysteine in serum for vitamin B12) are useful to detect iron and vitamin B12 deficiency as their plasma concentrations are not fully related to their nutritional status (e.g. functional vitamin B12 deficiency) $[128,129]$.

In addition, some studies have demonstrated high folate levels in patients associated with the high folate 


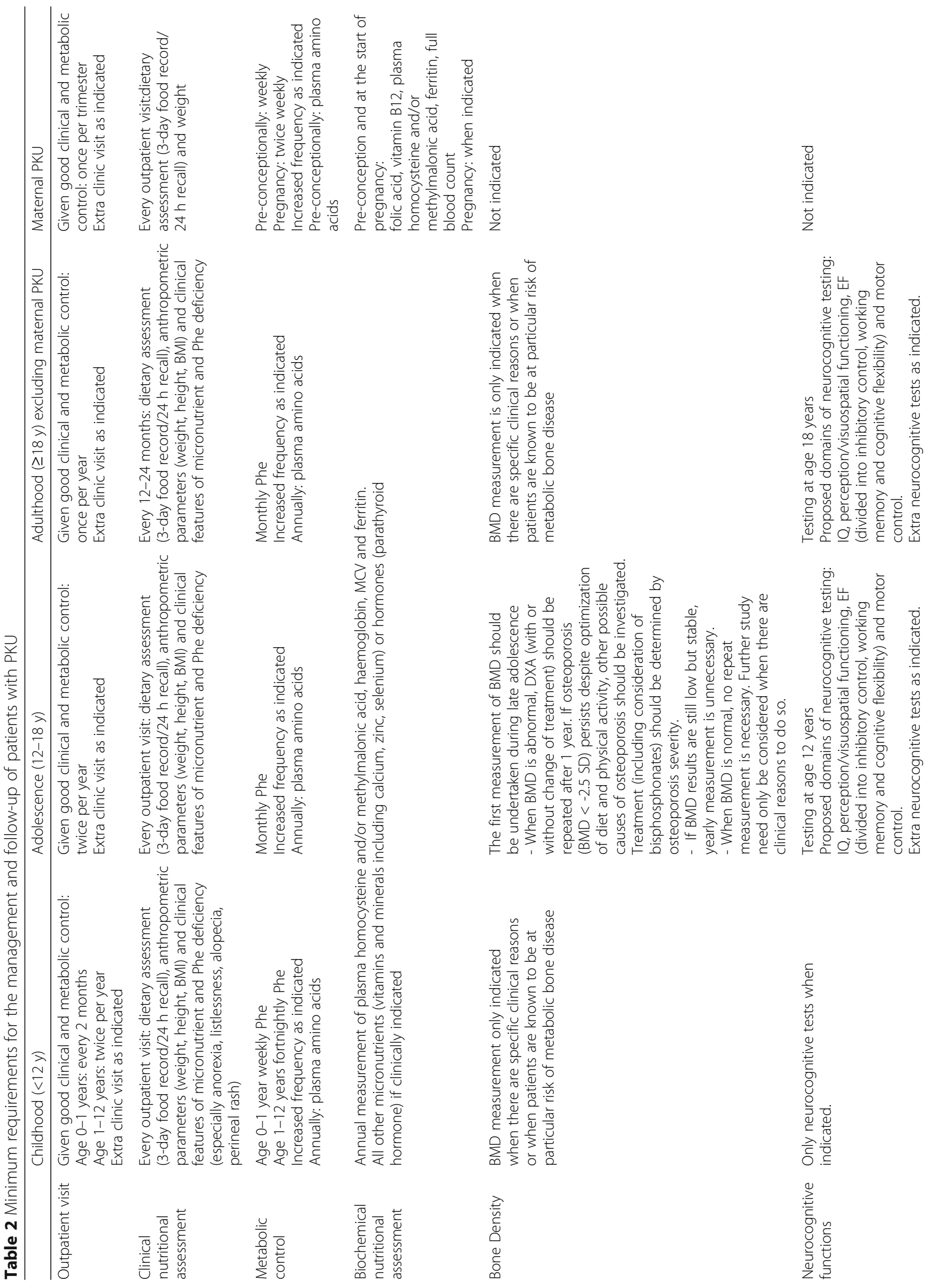




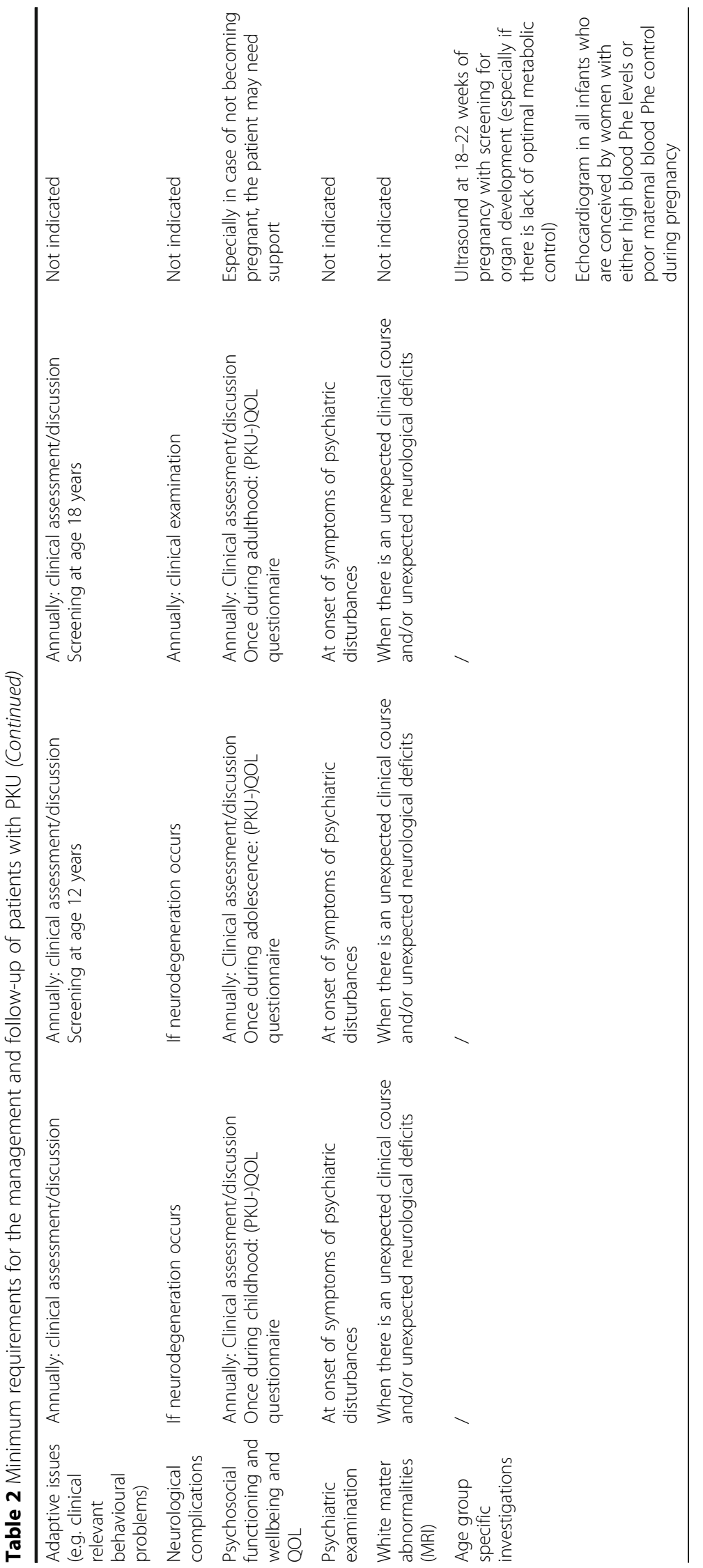


content of Phe-free L-amino acid supplements with added vitamins and minerals $[118,127,130]$. The longterm consequences of folate overload in PKU have not been assessed. Deficiencies of other micronutrients are rarely reported.

The nutritional follow-up requires the monitoring of anthropometry, body mass index (BMI), clinical signs of nutrient deficiency, nutrient intake and biological biomarkers to detect subclinical micronutrients excess or deficiencies. Accessing information about frequency and amount of L-amino acid supplements prescribed and delivered to a patient's home will give some indication about patient adherence, but still does not guarantee that products are consumed.

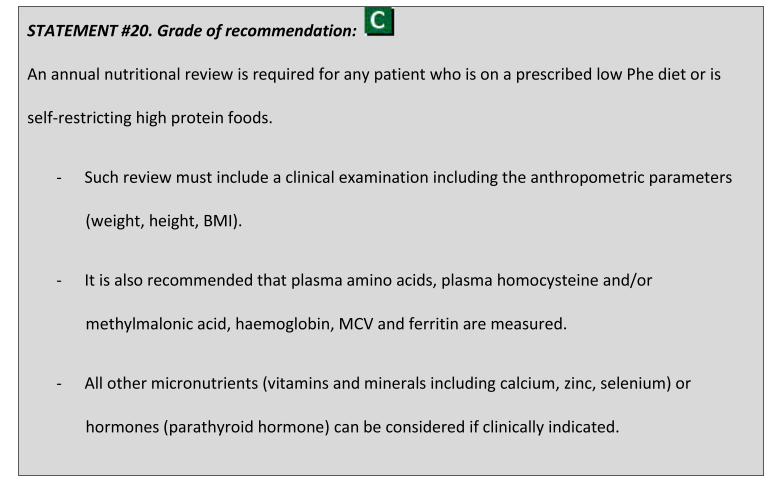

\section{Bone density}

The main factors influencing bone density are calcium and vitamin D status, the quality of bone proteins, physical activity, endocrine status, genetic and environmental factors.

\section{Osteopenia and PKU}

Osteopenia and osteoporosis in PKU has been described for many years. Definitions of osteopenia and osteoporosis are highly heterogeneous between studies and do not align with World Health Organization (WHO) standards and the International Society for Clinical Densitometry (ISCD) positions on bone mineral density (BMD) measurement [79]. According to the ISCD, fracture history must be assessed alongside BMD Z-score before diagnosis can be made [131]. In adult patients, WHO guidelines require $\mathrm{T}$-scores to diagnose osteopenia (T-score between -1 and -2.5 ) or osteoporosis (T-score below -2.5) [132]. In all patients Z-scores can be used, except in males older than 50 years and postmenopausal women in which the use of T-scores is advised.

There have been 3 systematic reviews on bone density in PKU: Enns et al. [5] (9 papers published after the year 2000), Hansen et al. [133] (16 papers) and Demirdas et al. [79] (13 papers) [5, 79, 133]. Enns et al. [5] found a sub-optimal outcome for bone health in PKU in all 9 studies. Hansen et al. [133], on a meta-analysis of 3 papers, showed a significantly lower spine BMD $\left(0.100 \mathrm{~g} / \mathrm{cm}^{2}\right)$ in 67 subjects with PKU compared to 161 controls. These papers included early and late treated PKU patients; only 1 corrected for reduced height. Demirdas et al. [79] performed a meta-analysis with only ETPKU patients. Mean total body (3 studies; $n=133$ ), lumbar spine ( 7 studies; $n=247$ ), and femoral hip (2 studies; $n=78$ ) BMD Z-scores in patients with PKU were lower than in their healthy peers, but well within the normal reference range, respectively $-0.45(95 \% \mathrm{CI}-0.61,-0.28) ;-0.70$ (95\% CI $-0.82,-0.57) ;-0.96$ (95\% CI $-1.42,-0.49)$ [79].

\section{Fracture risk in PKU}

An increased fracture risk has been infrequently described [134].

\section{Pathophysiology of osteopenia in PKU}

Nutritional factors and osteopenia in PKU Earlier studies described calcium and vitamin D deficiencies $[135,136]$, but the calcium and vitamin $\mathrm{D}$ content of current Phe-free L-amino acid supplements exceed requirements. Pérez-Dueñas et al. [73] showed a positive correlation between BMD and mineral intake and concluded that the correct intake of Phe-free L-amino acid supplement was necessary for bone mineralization. They found, however, that vitamin D supplements improved BMD in a cohort of patients with inadequate intake $(n=6 / 28)$ [135]. Despite adequate calcium and vitamin D content of Phe-free L-amino acid supplements, osteopenia is still identified in patients on strict low Phe diet and good metabolic control [137]. Patients with PKU also have an increase of calciuria, demonstrating no calcium deficiency [138]. Therefore, micronutrient intake is not the only causative factor of bone disease in PKU. Interestingly, docosahexaenoic acid (DHA) deficiency has also been associated with osteopenia in PKU [137].

The severity of PKU Osteopenia has not been observed in untreated MHP $[5,131,139,140]$. It has been described in classical PKU with various calcium metabolism profiles. In patients with classical PKU and poor diet, osteopenia was associated with an increase in parathyroid hormone (PTH) and alkaline phosphatase activity, both of which are related to calcium or vitamin D deficiency $[141,142]$. However, even classical PKU patients on strict diet with normal alkaline phosphatase and PTH activities may have osteopenia associated with osteoporosis pathophysiology.

Alteration of bone metabolism The metabolic profile of calcium metabolism in PKU patients is identical to that observed in classical osteoporosis (normal blood 
calcium, phosphorus, alkaline phosphatase and PTH associated with an increase of calciuria and C-terminal telopeptide). Demirdas et al. [79] reported that bone turnover results were ambiguous and that it is not clear from studies whether bone formation is decreased or bone resorption is increased. This may be partly due to heterogeneity in both markers and populations with regard to age [79].

Natural protein intake Bone health also depends on the quality of its protein structure as evident by the bone fragility observed in osteogenesis imperfecta. The impact of overall protein status, including the biological value of intact protein versus Phe-free $\mathrm{L}$-amino acid supplements and the percentage of protein derived from natural protein, is often not considered in studies [79]. Miras et al. [137] described 43 patients with classical PKU on a strict low-Phe diet, $14 \%$ of whom had mineral bone disease (MBD). The main difference between the group with and without MBD was the natural protein intake $(14.33+/-8.95 \mathrm{~g} /$ day in the group with MBD vs. $21.25+/-20.85$ in the group without MBD) [137]. Solverson et al. [143] showed an improvement of bone density in a group of mice treated with a low-Phe glycomacropeptide compared with Phe-free L-amino acid supplement [143], and Miras et al. [137] identified an absence of bone disease in 12/12 PKU patients treated by BH4, which allowed a higher natural protein intake [137].

Practically an adequate intake of calcium and vitamin $D$, regular exercise and optimization of natural protein intake must be ensured. We suggest follow-up of BMD during late adolescence (statement \#22), although there is no sound research data suggesting follow-up by DXA or other methods.

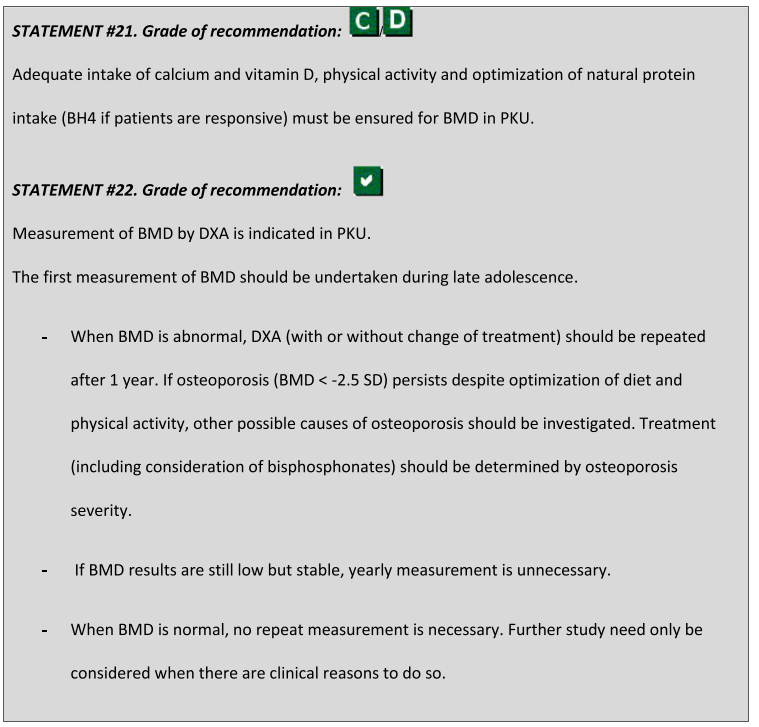

\section{Brain magnetic resonance imaging}

PKU is associated with the occurrence of white matter (WM) abnormalities (WMA) on magnetic resonance imaging (MRI) in both early and late treated patients [144-146]. The pattern of WM involvement in ETPKU is characterized by patchy or diffuse symmetrical lesions of deep and periventricular WM (occipito-parietal, frontal, temporal) appearing as signal hyper intensity on T2-weighted and FLAIR sequences and, in a minority of subjects, as signal hypo intensity in T1weighted sequences. Several controlled cross-sectional studies (with 21 to 77 PKU patients per study) showed that the extent and severity of WMA appear to be moderated by patient age and/or dietary adherence (as reflected by blood Phe levels), with older age and/or higher Phe levels associated with increased white matter involvement [147-153].

Whether these lesions have any clinical impact is unclear and the mechanisms involved in their pathogenesis are not known. No WMA in subjects with blood Phe levels $<300-600 \mu \mathrm{mol} / \mathrm{l}$ have been reported [49, 97-100]. Weglage et al. [49] found no WMA in 31 PKU adolescent and adult patients with untreated Phe levels $<600 \mu \mathrm{mol} / \mathrm{l}$ [49]. Bick et al. [97], Kono et al. [98], Lou et al. [99] and Manara et al. [100] found no WMA in respectively 2 PKU adults with (lifetime) Phe $<360 \mu \mathrm{mol} / \mathrm{l}$ [97], 7 PKU children and adolescents with (concurrent) Phe $<68$ $514 \mu \mathrm{mol} / \mathrm{l}$ [98], 2 PKU adolescents with Phe 200$300 \mu \mathrm{mol} / \mathrm{l}$ during childhood [99], 8 PKU adolescents with concurrent Phe $<400 \mu \mathrm{mol} / \mathrm{l}$ or mean Phe-year $<460 \mu \mathrm{mol} / \mathrm{l}[100]$.

However, other factors are involved as suggested by the occurrence of WM variation (improvement or worsening) in patients who did not change their Phe values, and the wide variability of WM involvement under similar value of blood and brain Phe [149, 150].

WMA are reported to be reversible. Two controlled studies [154, 155] showed an improvement of WMA (3 to 6 months) after lowering of blood Phe levels. Cleary et al. [154] reported improvement was primarily in those with reduced Phe levels $<900 \mu \mathrm{mol} / \mathrm{l}$ and scans improved in all 5 patients with reduced Phe levels $<400 \mu \mathrm{mol} / \mathrm{l}$. White et al. [155] also found improvements in $12 \mathrm{PKU}$ patients lowering their Phe levels from a mean of 653 (322) $\mu \mathrm{mol} / \mathrm{l}$ to 409 (256) $\mu \mathrm{mol} / \mathrm{l}$ [155]. Similar results come from single cases and small cohort studies $[97,156,157]$.

Current neuroimaging techniques are not useful in monitoring the clinical outcome for ETPKU patients. Neuroimaging examinations should be reserved for those patients presenting with an atypical clinical course and/or unexpected neurological deficits or for research purposes. 


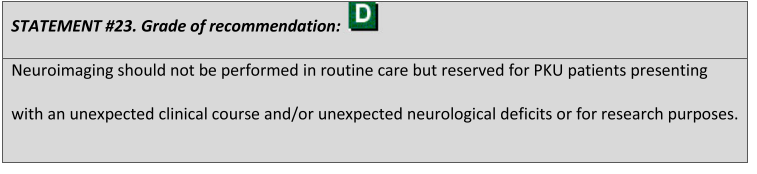

\section{Neurocognitive functioning}

PKU patients have an increased risk of developing neurocognitive problems [52, 53, 95]. Gassio et al. [158] demonstrated more school problems in ETPKU than control subjects, probably related to the disturbed cognitive functions observed. Although the majority of ETPKU individuals have educational and professional achievements similar to their non-PKU siblings, they have more pronounced problems in social functioning and emotional wellbeing [70, 159]. The clinical relevance and the relationship to metabolic control need to be established in future research. Routine neurocognitive evaluations should be performed at 12 and 18 years of age in all patients. This corresponds with changes in treatment targets for blood Phe or life changes (e.g. change of school, living situation, job, transfer to adult clinic.). This recommendation will provide baseline data about neurocognitive functioning prior to any relaxation of blood Phe levels at the age of 12 years or at the time patients are starting their adult life. Referral to a (neuro) psychologist is strongly recommended if risk factors apply as stated in statement \#24.

Supplying treating centers with the correct PKU profile for testing neurocognitive capacities in PKU patients for routine care at 12 and 18 years is still a challenge. In short, there are no PKU specific tests available to measure neurocognitive functions. While which test to use is largely a professional's choice and/or centre dependent, the target should be a comprehensive neuropsychological assessment exploring cognitive performance across different domains. Reminding this, literature shows many aspects of neurocognitive functioning in PKU patients for which, at some point, phenylalanine-related impairments have been shown. These include perceptual skills, visuospatial abilities, and fine motor control (for an overview, see Janzen and Nguyen [160]). Whereas for these aspects of cognition, impairments were shown relatively consistently, there are other domains such as language, verbal fluency, and long-term memory for which impairments were shown incidentally. The most consistent phenylalanine-related impairments have been observed in the domain of executive functioning (EF, e.g. inhibitory control, working memory, cognitive flexibility) (for an overview, see Christ et al. [161]). The level of complexity of the tasks that were used in neuropsychological assessments (in other words: the level of executive control that was required) seems to be a determining factor in whether or not impairments will be observed. Therefore, it is important that any form of neuropsychological assessment that will be chosen for the monitoring of PKU-patients captures these different levels of complexity. In young and young adult PKUs the assessment of some complex cortical functions, such as EFs (reasoning, planning, flexibility, and monitoring), visuo-motor coordination and speed of processing may be a sensitive tool in detecting possible neuropsychological impairment. However, there are some issues to be solved in research setting before we can introduce EF in routine clinical practice 1) the consistency of EF alterations in serial evaluations across different ages; 2 ) the predictive value of $\mathrm{EF}$ alterations with respect to later neurocognitive functioning and real life adaptation. Knowing all these issues and the time consuming aspects for staff members of these test, it will be important to continue to study links with instruments that have already shown to be more or less sensitive in picking up phenylalanine-related impairments in PKU [162].

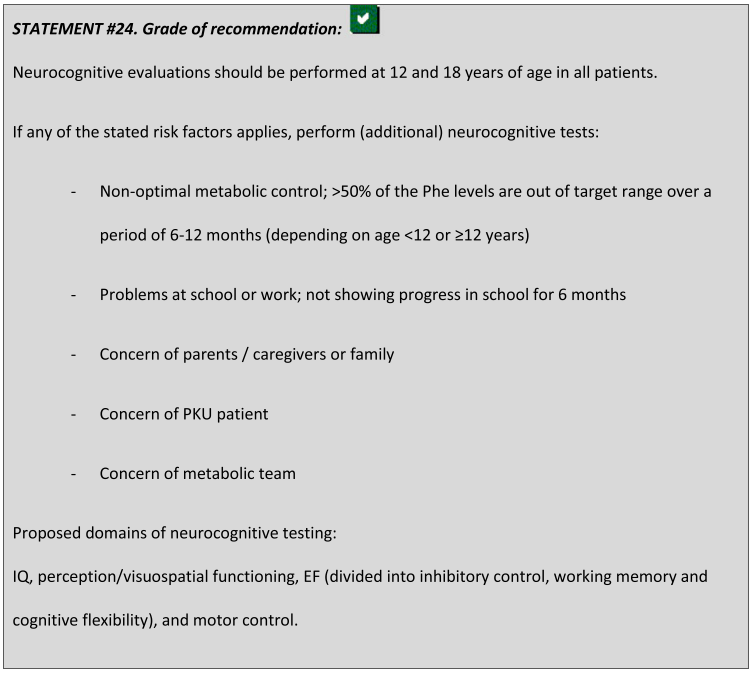

\section{Psychosocial functioning}

Studies evaluating the HRQoL of patients with ETPKU demonstrated a normal HRQoL compared to the general population [56,62,163,164], with the exception of 1 study reporting a lower score on the cognitive domain in adults [165], and 1 study demonstrating a lower HRQoL in a group of Italian children [166]. This contrasts with the view of patients and professionals who experience or observe stress associated with the burden of the diet. Normal HRQoL results may be due to the use of generic questionnaires or questionnaires aimed at the chronically ill but do not address the specific problems experienced by patients with PKU. Recently, a PKU specific HRQoL questionnaire has been developed and has been demonstrated to reliably assess the multifaceted impact 
of PKU on patients of different age groups [57]. Bosch et al. [58] showed good HRQoL in 306 PKU patients and 253 parents using this PKU specific questionnaire. Negative impacts of PKU on a patient's life, in particular the emotional impact of PKU and its management (anxiety about blood Phe levels, guilt related to poor adherence to dietary restrictions or Phe-free amino acid supplement intake) was found by the PKU specific HRQoL across all age groups [58].

There are no clinical studies available about the utility of measuring psychosocial functioning in PKU but its usefulness has been studied in other diseases. Measurement (HRQoL instruments) and discussion of psychosocial functioning during clinic visits significantly increased dialogue about psychosocial and emotional function in cohorts of adults, and in children with cancer [167-169], without increasing the duration of the consultation [167, 169]. In other conditions, such as adults with cancer and children and adolescents with diabetes, improved psychosocial outcomes were demonstrated $[167,169,170]$, but de Wit et al. showed in diabetes that improvements dissipated after 1 year when measurement and discussion about psychosocial function and wellbeing did not occur [171]. One study evaluating the effect on metabolic control in children with diabetes could not demonstrate improvement [170]. Considering that measurement of psychosocial functioning is usefull in other diseases, this could also be applied to PKU.

In PKU, for evaluation of HRQoL we advise using the PKU QoL questionnaires in addition to generic questionnaires.

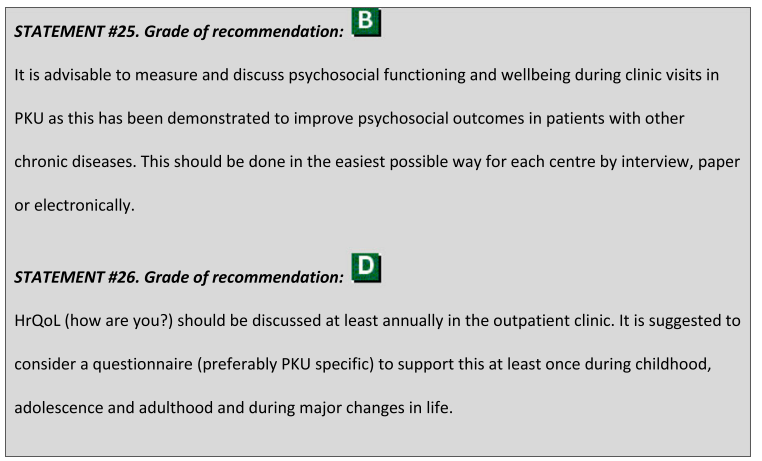

\section{Mental health problems in early treated PKU}

Determining the impact of PKU on mental health is difficult. One of the reasons for this is that different terminology is used e.g. behavioural difficulties, mental health, adaptive issues, and psychiatric symptoms. Adaptive behaviour is more commonly used and this is defined as a collection of conceptual, social and practical skills necessary to function appropriately in daily life. In addition, studies have used different questionnaires to assess mental health. Studies using the Child Behaviour Checklist (CBCL) in ETPKU patients of various ages, report differences in mainly internalizing problems such as social problems and withdrawal, anxiety/depression, poor attention and low self-esteem compared to the normal population [172-176]. However, when these PKU patients were compared to another chronic disorder of childhood, Diabetes Mellitus, no significant differences were found [173, 175]. While Weglage et al. [175] found no correlation between Phe values and CBCL scores, results of Jahja et al. [177] demonstrated that concurrent Phe was correlated to both internalizing and externalizing behavioural problems in children [175, 177]. Jusiene et al. [174] demonstrated that parental emotional coping accounts for $38 \%$ of the variance of internalizing problems. The reported internalizing symptoms in ETPKU are mainly attributed to having a chronic illness.

Smith et al. [178] demonstrated an increased prevalence of deviant behaviour strongly related to Phe values in a cohort of 544 PKU patients aged 8 years and 1088 controls. Burgard et al. [179] found more moderate psychiatric disturbances in $60 \mathrm{PKU}$ adolescents compared to 191 age matched controls, although these seemed to be more associated with the chronic condition than with the Phe level. Other studies using personality inventories and depression inventories found no significant differences in mental health between PKU patients and controls or norm scores [112, 180, 181].

Arnold et al. [182] reported a high incidence of attention problems from a chart study, with a strong relationship to Phe levels in the previous year. Twenty-six per cent of the PKU patients used attention deficit hyperactivity disorder (ADHD) medication compared to 6.5\% of diabetes type 1 patients (and 5\% of the normal population), mostly without formal ADHD evaluation by a psychologist [182]. Lowering Phe by starting BH4 treatment (without dietary adjustments) seemed to decrease ADHD symptoms in 38 patients aged $>8$ years [183].

Baieli et al. [184] found no patients with autism spectrum disorder in 62 ETPKU patients compared with 2 patients with autism spectrum disorder in 35 late treated PKU patients [184].

In adults with early and continuously treated PKU, there is a lack of evidence about mental health issues. Results from Jahja et al. [177] showed that adult PKU patients presented with more internalizing behavioural problems compared to controls. The Phe levels during childhood were associated with the internalizing behavioural problems [177].

To summarize, in ETPKU children there is an association of internalizing symptoms such as anxiety and depression with elevated concurrent and lifetime Phe levels. The impact of early treated PKU on mental health is most likely to be multifactorial, associated with chronic illness, 
persistently elevated Phe values, parental coping strategies and executive function deficits. PKU does not seem to enhance the autistic vulnerability in early treated subjects. Whilst studies have detected some increase in symptoms (e.g. anxiety) there is no strong evidence of increased psychiatric disease.

Because of the reported adaptive (behavioural and social) issues in ETPKU, it is important that behavioural screening is incorporated into the follow up of PKU. This enables patients to be referred to the appropriate services should severe difficulties be observed. Although there are many tests, the test choice is largely a professional preference and/or centre dependent.

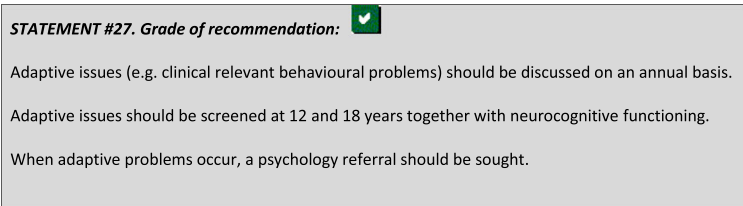

\section{Oxidative stress}

Oxidative stress is described in PKU patients and in PKU animal models as in many (neurodegenerative) disorders. In PKU it could be of importance in our understanding of cerebral PKU pathophysiology. There is clear data suggesting oxidative stress is related to poor metabolic control [101, 185] and micronutrient deficiencies (selenium, zinc, co-enzyme Q10 and perhaps L-Carnitine) [186, 187].

Due to the lack of clinical data linked to anti-oxidant status, no biochemical monitoring is proposed. Good blood Phe control appears to be important in reducing oxidative stress.

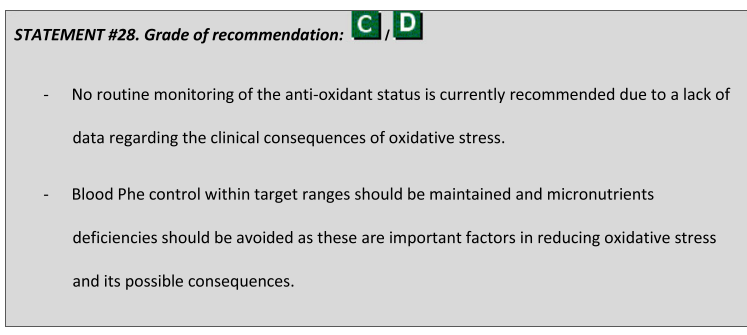

\section{Dietary treatment}

Dietary treatment is the basis of PKU management. It consists of 3 parts: natural protein restriction, Phe-freeL-amino acid supplements, and low protein food. Although we have longstanding experience with dietary treatment, it is only in recent years that there is more scientific evidence to support practice, but there remain gaps in several key areas.

\section{Natural protein restriction}

Phenylalanine is an indispensable, aromatic L-amino acid. It is essential for protein synthesis [188] and so must be provided in an amount that supports growth and tissue repair during childhood, and tissue repair in adulthood while keeping plasma Phe concentrations within recommended ranges [189].

\section{Requirements for Phe}

In order to promote protein synthesis, it is important to give the maximum amount of natural protein tolerated [190]. In PKU, the individual dietary Phe tolerance is influenced by many factors: severity of PKU, net protein catabolism-synthesis ratio, energy intake, dosage and distribution of Phe-free L-amino acid supplements, and target blood Phe concentrations. The individual Phe tolerance should be pragmatically determined, as minor increases of Phe intake may not necessarily affect blood Phe concentrations [191]. The Phe tolerance is defined as the amount of Phe per $\mathrm{kg}$ of body weight or $\mathrm{mg} /$ day that maintains blood Phe concentrations within the target range. This may also be described as natural protein tolerance expressed as $g /$ day. In PKU, generally Phe tolerance/ requirements per $\mathrm{kg}$ of body weight are highest in early infancy ranging from $55 \mathrm{mg} / \mathrm{kg} /$ day at 0-3 months of age to $27 \mathrm{mg} / \mathrm{kg} /$ day at 12 months [192]. After the age of 1 year, there is a slow and steady decline in tolerance per $\mathrm{kg}$ of body weight, and even from the early times of treating PKU with diet it has been recognized that children with classic PKU usually only tolerate between 200 and $500 \mathrm{mg}$ Phe/day. Patients with a milder form of PKU (untreated blood Phe concentrations less than 1000$1200 \mu \mathrm{mol} / \mathrm{l}$ ), usually tolerate $\geq 500 \mathrm{mg} /$ day of dietary Phe. By comparison, in non-PKU, the third US National Health and Nutrition Examination Survey (NHANES III) demonstrated that mean daily dietary Phe intakes for all life stages and gender groups was as high as $3400 \mathrm{mg} /$ day (http://www.cdc.gov/nchs/nhanes.htm). A clear relationship between Phe tolerance at 2 years of age and at 10 years of age was found [40], although it is unknown how this tolerance relates to the tolerance in older patients with PKU aiming to achieve a target blood Phe of 120 to $600 \mu \mathrm{mol} / \mathrm{L}$. There is clearly a need to evaluate the Phe tolerance of all patients periodically, but particularly at the times of rapid growth, changes in body composition or use of different treatment modalities (e.g. BH4). For patients responsive to $\mathrm{BH} 4$, it is likely that natural protein tolerance may double [193] or quadruple $[193,194]$. However, there still may be over restriction of dietary Phe intake with low Phe diets (with and without BH4 treatment). Both MacLeod et al. and van Rijn et al. demonstrated in 8 (not clearly well-controlled patients) and 6 (well-controlled) patients that Phe intake could increase substantially without changing the blood Phe 
concentrations significantly [190, 195]. It has also been shown that Phe intake can be increased with a higher dose of Phe-free L-amino acid supplements [196, 197]. Finally, regular assessement of actual Phe intake, compared with the prescribed amount is important in helping to define actual Phe tolerance as some patients eat more Phe than their prescribed amount without affecting blood Phe control [103, 198].

\section{Phe deficiency}

Reports of symptomatic Phe deficiency still appear in the literature [80, 81]. Symptoms include: anorexia, listlessness, alopecia, perineal rash, poor and variable growth in preschool children and even death, while biochemical abnormalities include generalized aminoaciduria. Unnecessary dietary restrictions should therefore be avoided.

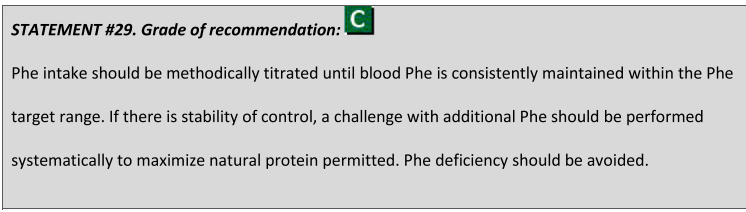

\section{Protein requirements}

In most patients, it is likely that precursor free L-amino acids will supply 52 to $80 \%$ of the total protein intake [199-201]. However, the optimal amount of L-amino acids has caused extensive debate and is undetermined. The recent Cochrane review concludes there is insufficient data to reach any conclusions regarding the dosage of Phe-free L-amino acid supplements in the treatment of PKU [201]. Considerations for any recommendations for the dosage of Phe-free L-amino acid supplements intake should include:

1) the protein recommendations for the healthy individual;

2) studies on growth in PKU;

3) inefficiency associated with the utilization of L-amino acids;

4) any functional effects of L-amino acids;

5) any side effects associated with dosage of L-amino acids;

6) natural protein tolerance (i.e. natural protein + Phe-free L-amino acid supplements = total protein intake). The latter will vary according to the severity of PKU, age, clinical condition (e.g. presence of malnutrition/malabsorption), growth, and use of additional treatment options such as $\mathrm{BH} 4$.

In addition, the current estimates of protein requirements are defined as the lowest level of dietary protein intake that will balance the losses of nitrogen from the body, and thus maintain the body protein mass in persons at energy balance with modest levels of physical activity [202]. Such a definition does not necessarily identify the optimal intake for health, which is less quantifiable [203], or any specific requirements in clinical situations. To make it even more complex, only the need for L-amino acids as a whole is discussed, but in fact each amino acid (especially indispensable) deserves individual attention, as many of them are large neutral amino acids (LNAA) that may play a specific role in PKU pathophysiology [2, 204].

\section{Protein requirements for growth/physiological needs}

A number of observational studies (infants to adults) have investigated the Phe-free L-amino acid supplements dosage necessary for optimal growth in PKU [192, 196, 197, 205-220]. They have demonstrated that growth in PKU is mainly satisfactory if the total protein intake (largely given as Phe-free L-amino acid supplements) meets or is above the general population recommendations. In the published studies, national recommendations were commonly based on the FAO/WHO/UNU 1985 safe levels of protein intake [221]. However, recently, the FAO/WHO/UNU 2007 has reduced the safe levels of protein intake (in infants under 1 year by approximately 25 to $27 \%$, children $1-5$ years by 17 to $21 \%$ and children $6-10$ years by 8 to 13\%) (Table 3) [202]. No studies have examined growth in PKU on this level of total protein intake so these requirements should not be used until there is published data to support such a low protein intake in PKU. Many centres in Europe and beyond prescribe L-amino acids/total protein between 2 and $3 \mathrm{~g} / \mathrm{kg} /$ day in infants aged 0-1 y; children aged 1-10 y; $1.5-2 \mathrm{~g} / \mathrm{kg} /$ day and >10y: $1 \mathrm{~g} / \mathrm{kg} /$ day (Table 4) [6, 207]. This data was confirmed by a survey of 63 PKU centres from 18 countries, demonstrating that prescription patterns of total protein intake was influenced by country and location in Europe (e.g. South, North, West, East Europe) [222]. In general no more than $20 \%$ of energy should be supplied as protein [223].

\section{Digestibility and bioavailability of L-amino acids}

There is insufficient data about the digestibility and bioavailability of L-amino acids [203]. L-amino acid requirements in PKU have not been determined under various conditions such as inadequate energy intake (absorbed L-amino acids may be utilized via catabolism to provide adenosine triphosphate usually referred to as ATP, rather than for body protein synthesis) or on a very low natural protein intake. L-amino acids do not require digestion and are directly available for absorption by the small intestine [224]. This leads to rapid absorption [225, 226]. Not only do plasma amino acids rise more quickly and to higher concentrations but also fall more quickly than whole protein sources like casein [226]. In addition 
Table 3 A comparison of the protein intakes recommended by FAO/WHO/UNU 1985 and FAO/WHO/UNU 2007 report

\begin{tabular}{|c|c|c|c|}
\hline Age & $\begin{array}{l}\text { FAO/WHO/UNU } \\
2007 \text { Report }\end{array}$ & $\begin{array}{l}\text { FAO/WHO/UNU } \\
1985 \text { Report }\end{array}$ & $\begin{array}{l}\text { \% change between } \\
2007 \text { and } 1985 \\
\text { safe levels of } \\
\text { protein intake }\end{array}$ \\
\hline \multirow[t]{2}{*}{ Years } & Safe level (+1.96SD) & Safe level (+1.96SD) & \\
\hline & g/kg/day & g/kg/day & \\
\hline 0.5 & 1.31 & 1.75 & $-25 \%$ \\
\hline 1 & 1.14 & 1.57 & $-27 \%$ \\
\hline 1.5 & 1.03 & 1.26 & $-18 \%$ \\
\hline 2 & 0.97 & 1.17 & $-17 \%$ \\
\hline 3 & 0.90 & 1.13 & $-20 \%$ \\
\hline 4 & 0.86 & 1.09 & $-21 \%$ \\
\hline 5 & 0.85 & 1.06 & $-20 \%$ \\
\hline 6 & 0.89 & 1.02 & $-13 \%$ \\
\hline 7 & 0.91 & 1.01 & $-10 \%$ \\
\hline 8 & 0.92 & 1.01 & $-9 \%$ \\
\hline 9 & 0.92 & 1.01 & $-9 \%$ \\
\hline 10 & 0.91 & 0.99 & $-8 \%$ \\
\hline \multicolumn{4}{|l|}{ Girls } \\
\hline 11 & 0.90 & 1 & $-10 \%$ \\
\hline 12 & 0.89 & 0.98 & $-9 \%$ \\
\hline 13 & 0.88 & 0.98 & $-10 \%$ \\
\hline 14 & 0.87 & 0.94 & $-7 \%$ \\
\hline 15 & 0.85 & 0.87 & $-2 \%$ \\
\hline \multicolumn{4}{|l|}{ Boys } \\
\hline 11 & 0.91 & 0.99 & $-8 \%$ \\
\hline 12 & 0.90 & 0.98 & $-8 \%$ \\
\hline 13 & 0.90 & 1 & $-10 \%$ \\
\hline 14 & 0.89 & 0.97 & $-8 \%$ \\
\hline 15 & 0.88 & 0.96 & $-8 \%$ \\
\hline
\end{tabular}

nitrogen retention [227] following ingestion of L-amino acids is less efficacious than with casein rich protein suggesting a less efficient transfer of L-amino acids into tissue and plasma proteins $[225,228]$. There is also a suggestion of increased oxidation when Phe-free Lamino acid supplements are taken in large single doses [229], but this may be reduced by small frequent doses of Phe-free L-amino acid supplements, particularly if given bound with intact protein [230].

Overall, it is well established that Phe-free L-amino acids supplements are associated with a lower biological efficiency compared with natural protein sources and some compensatory factor should be considered for this in the protein requirement recommendations. Some have suggested an additional $20 \%$ of L-amino acids should be provided to compensate for their inefficiency (Dutch guidelines, unpublished). This was based on several recommended safe levels of protein including FAO/WHO/UNU [221] and [202]. USA recommended dietary allowances (RDA) also proposes an adjustment of approximately $20 \%$ to compensate for losses due to digestibility and protein quality for mainly vegetarian diets, but their baseline protein requirements are higher than the 2007 safe levels of protein intake [202].We suggest providing an additional $20 \%$ of $\mathrm{L}$-amino acids to compensate for the 'digestible indispensable amino acid score' and also a further $20 \%$ of L-amino acids to optimize their impact on blood Phe control. This leads to a total of $40 \%$ additional L-amino acids, although the optimal dose for this function is undetermined.

For example, if a man with PKU with a body weight of $100 \mathrm{~kg}$ (ideal body weight $70 \mathrm{~kg}$ ) is allocated $6 \mathrm{~g} /$ day natural protein, the intake of $\mathrm{L}$-amino acids is calculated as follows: 70 (ideal body weight) $\times 0.8$ (safe level of protein intake) $=56 \mathrm{~g} /$ day total protein requirements.

To calculate the L-amino acid requirement: total protein intake $(56 \mathrm{~g} /$ day $)$ - natural protein intake $(6 \mathrm{~g} /$ day $)=50 \mathrm{~g} /$ day. This is corrected with an additional $40 \%$ of L- amino acids from the protein substitute $=50 \mathrm{~g} / \mathrm{day} \times 1.4=70 \mathrm{~g} /$ day.

Ideally, the protein requirements should be based on ideal body weight for height and age. This is particularly important for overweight and obese patients as their total protein intake may be particularly high if based on actual weight only [231].

\section{Functional effect of Phe-free L-amino acid supplements}

Protein substitutes should supply an adequate source of Phe-free indispensable L-amino acids. It is well established that they decrease blood Phe concentrations [115, 232-234]. It is also noted that blood Phe concentrations increase when patients do not take their Phe-free Lamino acid supplements as prescribed [232, 235]. Higher doses of Phe-free L-amino acid supplements are associated with improved Phe control [233] and a higher Phe tolerance $[196,197]$. Some of this has been attributed to improved anabolism associated with a higher dose of L-amino acids but it may also be related to the ability of specific individual LNAA (histidine, isoleucine, methionine, leucine, threonine, tryptophan, tyrosine and valine) within the supplements to alter Phe transport at gut epithelial level [236]. Within regular Phe-free Lamino supplements $35-50 \%$ of total $\mathrm{L}$-amino acids are supplied by LNAA's. Two randomized controlled trials on LNAA demonstrate that they significantly reduce blood Phe concentrations [235, 237, 238]. Large neutral and the cationic (lysine and arginine) L-amino acids cross the intestinal mucosa by a carrier protein similar to that of the blood brain barrier. In in vitro studies investigating intestinal epithelial transport, lysine, histidine, leucine and tyrosine significantly reduce Phe 


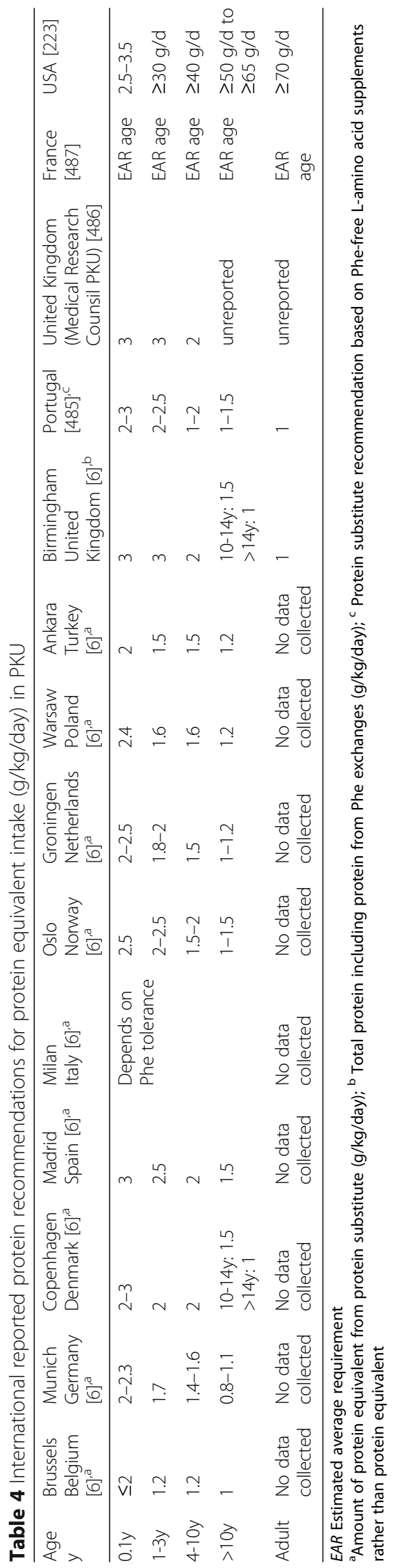


transport [236]. This suggests that the competition with the transport of Phe can be achieved by high concentrations of cationic L-amino acids and LNAA in the gut [236]. There is also evidence to demonstrate that LNAA block the transport of Phe across the blood brain barrier [239-242]. It is therefore established in PKU that L-amino acids within Phe-free L-amino acid supplements do more than provide replacement protein for growth and to maintain body protein mass. They have a role in blood Phe control, they inhibit the transport of Phe into the brain, and possibly via the gut too, and evidence suggests higher doses improve Phe tolerance. In athletes, although exercise is considered to have a major impact on protein metabolism and additional protein may be necessary to support global energy demands, we are unable to give a recommendation on any additional protein requirement for high level sports as this has not been studied in PKU.

\section{Adverse effects of Phe-free L-amino acid supplements}

Any side effects of Phe-free L-amino acid supplements should be considered. Phe-free L-amino acid supplements are hyperosmolar and so may cause gastrointestinal upset [231]. The osmolality of protein substitutes designed for children range from 600 to $2700 \mathrm{mosmol} / \mathrm{kg} \mathrm{H}_{2} \mathrm{O}$ depending on their dilution with water compared to 300$975 \mathrm{mosmol} / \mathrm{kg} \mathrm{H}_{2} \mathrm{O}$ for paediatric enteral feeding products based on milk protein (data from manufacturers data sheets). Abdominal pain, diarrhoea and constipation have been reported in a small series of young children [243]. Also, life-long and higher intake of Phe-free L-amino acid supplements is linked to proteinuria and decreased glomerular filtration rate in adults [199], although this has not been studied in a controlled way. Future research is needed to determine if this should be part of routine care.

Dental health may be affected by Phe-free L-amino supplements but few studies have examined dental health in PKU. One study reported no significant difference in dental caries between a group of children with PKU and controls with $75 \%$ of all children being caries free. However, they did identify more signs of tooth wear in PKU children, associated with the titratable acidity of flavoured Phe-free L-amino acid supplements (92.86$126.8 \mathrm{mEq} / \mathrm{l})$, which is significantly higher than unflavoured supplements $(4.18-14.0 \mathrm{mEq} / \mathrm{l})$ and coca cola (38.56 mEq/l) [244].

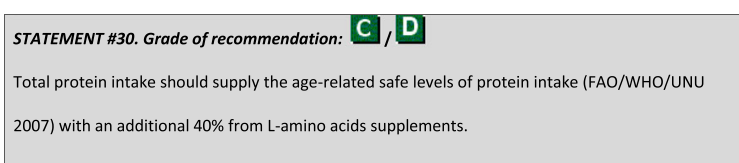

\section{Supplementation of L-amino acids}

In patients with PKU who requires severe restriction of natural protein intake, the provision of a suitable Phefree protein replacement/substitute is essential to prevent protein deficiency and optimize metabolic control. Protein substitutes are mainly sourced from Phefree L-amino acids and less commonly from low Phe glycomacropeptide protein. The Phe-free L-amino acid supplements should be evenly administered throughout the day $[103,233]$ to minimize losses of L-amino acids due to oxidation, and to help minimize fluctuations in blood Phe concentrations over a 24-h period. Therefore, we advise to divide the Phe-free L-amino acids into at least 3 equal portions throughout the day. The dosage and administration of Phe-free-L-amino acid supplementation should be managed by the metabolic dietitian and/or physician.

\section{Supplementation of Phe-free L-amino acids with added nutrients}

Many Phe-free L-amino acid supplements contain the addition of variable amounts of carbohydrate [245], vitamins, minerals, and long chain polyunsaturated fatty acids (LC-PUFA), with the aim of meeting the nutritional requirements for a product specific age targeted population. The aim is to ensure that the dietary needs for all vitamins, minerals and LC-PUFA are met when average dosages are prescribed for a specific age targeted population. However, higher or lower doses of Phe-free L-amino acid supplements may affect vitamin and mineral intake accordingly. Two longer term observational studies have reported the impact on micronutrient status of Phe-free L-amino acid supplements with added micronutrients $[118,246]$. The review by Robert et al. [120] on related studies addressing micronutrient status in PKU suggested that there have been fewer deficiencies since the mid1990s when the practice of adding vitamins, minerals and trace minerals to Phe-free L-amino acid supplements was increased.

\section{Balance of L-amino acids in supplements}

In the normal population, there are guidelines for Lamino acid scoring pattern for infants, children and adults [202, 203]. In infants it is considered that breast milk amino acid content is the best estimate of amino acid requirements, but data on requirements from 1 year to adults is unreliable [203] and therefore any requirements for children are estimated using a factorial approach based on L-amino acid requirements for maintenance and growth. The ideal L-amino acid profile of Phe-free L-amino acid supplements is not determined. 


\section{Presentation of Phe-free L-amino acid supplements}

For children over 12 months of age, Phe-free L-amino acid supplements are mainly presented as flavored/ unflavored powders (cans/pre-measured sachets) and ready to drink liquids (pouches, bottles and tetrapaks). Powders are designed to be used either as a gel/paste, drink or mixed with food. Additionally, low volume semi-solid weaning products, capsules and tablets are available. Adherence with Phe-free L-amino acid supplements is reported as a major issue, mainly associated with their bitter taste [189, 247, 248]. Failure to take the prescribed amount is linked with poor metabolic control [232, 235]. However, recent evidence suggests that improved taste, volume, presentation and availability (by home delivery) of existing preparations has improved long-term adherence, particularly in teenagers taking liquid Phe-free L-amino acid supplements $[246,249,250]$. Therefore, it is important that patients have a choice of suitable age appropriate Phe-free L-amino acid supplements.

\section{Transitioning of Phe-free L-amino acid supplements}

Many of the Phe-free L-amino acid supplements are designed for different age groups e.g. Phe-free L-amino acid infant formula, weaning and toddler products, or supplements aimed at school children, teenagers and adults. There are no published studies indicating the best way to support patients transitioning Phe-free Lamino acid supplements from one age group to another. It is established that neophobia is prevalent in children with PKU and many find change particularly difficult [251]. A staged, systematic approach carefully transitioning over products from one to another may be warranted but requires research.

\section{Alternative sources of protein substitute}

GMP is a low Phe protein source derived from whey protein, used as an alternative to Phe-free L-amino acid supplements in the treatment of PKU. Commercially available GMP protein still requires supplementation with a significant proportion of L-amino acids including tyrosine, tryptophan, histidine and leucine [252] and does contain some Phe. In PKU, human GMP research is limited. Short-term data from a small controlled study in older patients suggest that fasting Phe concentrations and blood urea nitrogen were significantly lower with GMP compared with Phe-free L-amino acid supplements [253]. There is also the suggestion that GMP lowers post-prandial concentrations of the appetite stimulating hormone ghrelin, and may help promote satiety [254]. So far, no long-term outcome data has been reported, and no information about nutritional status or GMP's safety in children less than 11 years of age has been published. It should not be considered as an alternative source of protein equivalent in children or pregnancy until more data is available.

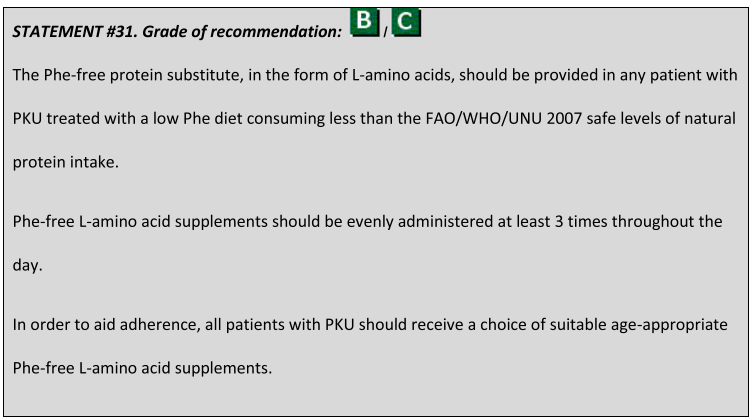

\section{Nutritional requirements}

Calorie requirement and energy expenditure

It is assumed that the energy requirement of patients with PKU is similar to healthy individuals, although there is very little supporting published data. Allen et al. reported that resting energy expenditure in children with PKU did not differ from that of normal children and data describing the energy intakes of patients would suggest that they are close to estimated average energy requirements [255]. Rohde et al. [191] found that patients consumed $95 \%$ of the RDA as agreed by the German speaking counties (DACH-RDA). MacDonald et al. [256] reported a mean energy intake of $105 \%$ of the estimated average requirement, and Rocha et al. [257] reported that patients with classical PKU consumed an additional $100 \mathrm{kcal} /$ daily than patients with mild PKU and $200 \mathrm{kcal} /$ daily more than patients with HPA. There is a growing number of reports of increased incidence of overweight and obesity in PKU [258], but in Portugal and the UK it has been shown to be similar to control groups [257] or general populations [77]. Although there is some association with poor adherence particularly in females with higher blood Phe concentrations [77, 258, 259], currently there is no direct correlation with the consumption of a high carbohydrate foods and overweight and obesity in patients with PKU.

Notwithstanding the importance of avoiding excess energy intake, it is equally important that age related average energy requirements are met for optimal dietary protein utilization, preventing catabolism (resulting in increases of blood Phe) [260-262]. Catabolism is defined as degradative metabolism that breaks down complex molecules as protein or lipids, releasing energy. Protein synthesis and catabolism are energy dependent and thus are sensitive to dietary energy deprivation. Insulin is secreted in response to carbohydrate (and protein) intake, promoting cellular uptake and use of L-amino acids. Energy and/or glucose depletion will result in L-amino acid (especially branched chain) breakdown (gluconeogenesis) 
to meet minimal glucose requirement, which can ultimately lead to a loss of metabolic control. When energy intake is decreased, protein required to maintain the same nitrogen retention is increased in proportion to the energy decrement [263-265].

Although it is aimed to give a percentage distribution of energy, carbohydrate, and fat similar to recommendations for a healthy population, in a low Phe diet, only 20 to $25 \%$ of energy is provided from fat $[266,267]$ (a typical omnivore diet will provide at least 35\% energy from fat). This is due to a low intake of fat/protein containing foods, and increasing carbohydrate to provide almost $60 \%$ of energy requirements [256, 257], with $15 \%$ from protein equivalent sources. Therefore, a strict low Phe diet is also low in $\alpha$-linolenic acid, arachidonic acid and without dietary sources of eicosapentaenoic acid (EPA) and DHA [268]. Evidence suggests that children with PKU have reduced concentrations of DHA in plasma and membrane phospholipids when compared to controls [266, 269-271]. Controlled trials with DHA \pm arachidonic acid supplements [272, 273] have led to improvement in LC-PUFA status. It is important that consideration is given to supplementation with EPA and DHA if these are not already added to the precursor-free L-amino acid supplement. The optimal dosage of DHA/EPA in children is not established but dosages between 180 and $500 \mathrm{mg}$ daily are provided by Phe-free L-amino acid supplements for children aged between 2 and $16 \mathrm{y}$.

\section{Micronutrient requirement}

Micronutrient intake should at least meet theoretical age-related reference nutritional intakes for the normal population. The main micronutrient sources are chemical and usually added to Phe-free L-amino acid supplements. The bioavailability of all micronutrients added to Phe-free $\mathrm{L}$-amino acid supplements is not well studied, and there is little longitudinal data on micronutrient status in patients with PKU following both strict and relaxed dietary regimens.

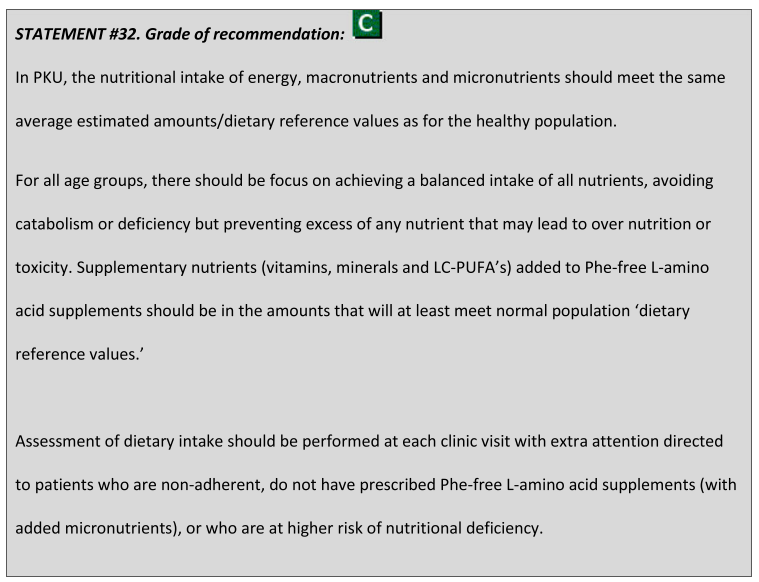

\section{Low protein foods, fruits and vegetables}

A number of low protein foods have been developed for PKU and are important for satisfying appetite and providing variety in a low Phe diet [274]. The availability of low protein foods is a key element in the successful application of a low Phe diet. Many regular basic foods such as bread, flour, and pasta based on wheat flour are not permitted in a low Phe diet because they contain too much natural protein. Instead they are replaced by low protein equivalent foods made from food starches (wheat, potato and maize starch). These special low protein foods should contain Phe $\leq 50 \mathrm{mg} / 100 \mathrm{~g}$ (equivalent to protein: $1 \mathrm{~g} / 100 \mathrm{~g}$ ) of dry product. They are an important source of energy, increase dietary variety and aid dietary adherence. However, they should contain no more energy, fat, carbohydrate or sugar than their equivalent natural protein containing foods. Although the energy contribution from these foods has not been formally reported, they may provide 35 to almost $50 \%$ of energy intake in severe PKU. All patients should have access to a choice of affordable basic low protein foods (e.g. bread, pasta, cereal, flour, egg and milk-replacements).

In PKU, most fruit and vegetables only yield $30-40 \mathrm{mg}$ Phe per $1 \mathrm{~g}$ of protein [275] compared with foods such as milk and cereals that contain $50 \mathrm{mg}$ Phe per $1 \mathrm{~g}$ of protein. There is evidence that fruits and vegetables (potatoes not tested) with a Phe content $<75 \mathrm{mg} / 100 \mathrm{~g}$ of food do not elevate plasma Phe concentrations [276]. In addition, vegetables containing Phe between 76 and $100 \mathrm{mg} / 100 \mathrm{~g}$ of food do not increase plasma Phe concentrations when eaten in small portions. Also use of similar 'free' fruits and vegetables by other countries has not been shown to adversely affect blood Phe control in short-term and longer-term studies [191, 277, 278]. Table 5 provides an overview of these papers.

Permitting fruits and vegetables without limitations with a Phe content $<75 \mathrm{mg} / 100 \mathrm{~g}$ of food will allow greater dietary variety and freedom and will aid dietary adherence. One exception is potatoes. They contain a variable Phe content and their effect on Phe control of their 'free' inclusion in the diet requires further testing.

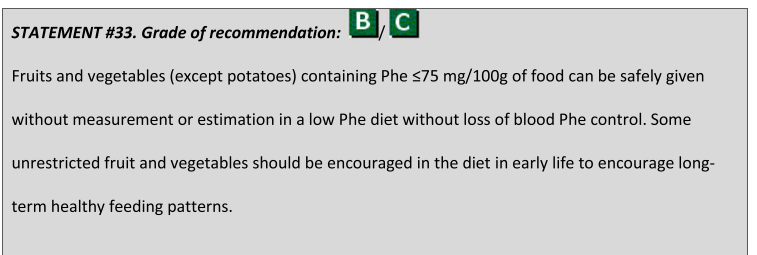

\section{Breast-feeding}

Breast-feeding offers several nutritional, psychological and practical advantages. Generally it is low in Phe 
Table 5 Evidence supporting free use of fruit and vegetables containing Phe $\leq 75 \mathrm{mg} / 100 \mathrm{~g}$

\begin{tabular}{|c|c|c|c|c|c|c|}
\hline Reference & $\mathrm{N}$ & Fruit/veg criteria & Study design & Change in Phe intake & Blood Phe control & $\begin{array}{l}\text { Grade of } \\
\text { evidence }\end{array}$ \\
\hline MacDonald et al. 2003 [276] & 15 & $\begin{array}{l}\text { Free use of fruit and veg } \\
\leq 75 \mathrm{mg} / 100 \mathrm{~g} \text { Phe } \\
\text { Not potatoes }\end{array}$ & $\begin{array}{l}15 \text { week systematic } \\
\text { challenge }\end{array}$ & $\begin{array}{l}\text { Mean Phe \# Approx } \\
50 \text { mg/day }\end{array}$ & $\begin{array}{l}\text { No impact on } \\
\text { Phe control }\end{array}$ & $2-/ C$ \\
\hline Rohde et al. 2012 [191] & 14 & $\begin{array}{l}\text { Free use of fruit and veg } \\
\leq 75 \mathrm{mg} / 100 \mathrm{~g} \text { Phe }\end{array}$ & $\begin{array}{l}2 \text { week randomised } \\
\text { cross-over -trial }\end{array}$ & $\begin{array}{l}\text { Mean Phe \# Approx } \\
50-60 \text { mg/day }\end{array}$ & $\begin{array}{l}\text { No impact on } \\
\text { Phe control }\end{array}$ & $1-/ B$ \\
\hline Rohde et al. 2014 [277] & 19 & $\begin{array}{l}\text { Free use of fruit and veg } \\
\leq 75 \mathrm{mg} / 100 \mathrm{~g} \text { Phe }\end{array}$ & 1 year follow up study & $\begin{array}{l}\text { Mean Phe \# Approx } \\
60-70 \text { mg/day }\end{array}$ & $\begin{array}{l}\text { No impact on } \\
\text { Phe control }\end{array}$ & $2-/ C$ \\
\hline Zimmermann et al. 2012 [278] & 50 & $\begin{array}{l}\text { Free use of fruit and veg } \\
\leq 100 \mathrm{mg} / 100 \mathrm{~g} \text { Phe Not } \\
\text { potatoes }\end{array}$ & $\begin{array}{l}\text { Up to } 3 \text { year follow up } \\
\text { study }\end{array}$ & Unreported & $\begin{array}{l}\text { No impact on } \\
\text { Phe control }\end{array}$ & $2-/ C$ \\
\hline
\end{tabular}

$N$ number of patients

(46 mg/100 mL), contains long chain polyunsaturated fatty acids and many non-nutritional bioactive compounds, is convenient, reduces the number of infant bottles, and provides the mother some control over the feeding process [279]. Many studies have reported satisfactory blood Phe control and growth with its use [280-285] and it is advocated by many PKU centres [6].

There have been different approaches to breast-feeding technique. Many have reported demand breast-feeding on the principle of giving a measured volume of a Phe-free infant formula before breast feeds, so reducing stimulation and production of breast milk, thus reducing breast milk and Phe intake. Blood Phe concentrations are used to determine the volume of Phe-free infant formula [279, 284]. Van Rijn et al. [285] alternated feeds between breastfeeding and Phe-free L-amino acid infant formula bottlefeeding and was able to achieve acceptable blood Phe control.

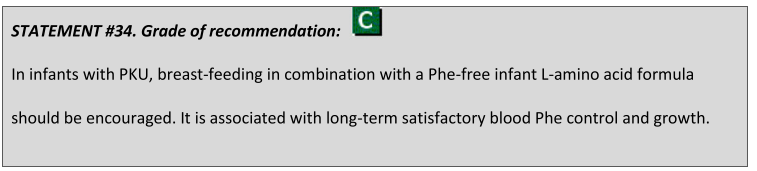

\section{Aspartame}

Aspartame (E951) (1-aspartyl-1-Phe methylester) is an intense sweetener derived from a dipeptide composed of Phe (50\%), aspartic acid (40\%) and methanol (10\%) [286, 287]. It is a source of Phe. It is added to soft drinks, chewing gums, sweets, desserts, jelly and table top sweeteners. The approximate amounts of aspartame in foods are: a $360 \mathrm{ml}$ can of diet coke is $130 \mathrm{mg}, 1$ portion aspartame flavoured jelly is $40 \mathrm{mg}, 1$ teaspoon of artificial sweetener $15-20 \mathrm{mg}$ and 1 piece of sugar-free chewing gum $5 \mathrm{mg}$. In the 1980's/1990's, many small intervention (controlled and uncontrolled) studies examined the impact of aspartame in patients with PKU, with most studies demonstrating a small, but consistent, increase in blood Phe concentrations with aspartame [287-295]. Fortunately, there are many other artificial sweeteners available that do not contain Phe. These artificial sweeteners include sucralose, saccharin, and acsesulfame potassium, so aspartame is easier to avoid in a low Phe diet.

The sweetener, neotame, also contains Phe, but the availability of Phe is largely reduced due to inability to break down the peptide bond between aspartic acid and Phe [296].

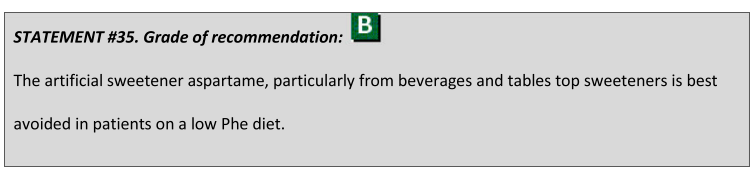

\section{Tyrosine supplementation}

In PKU, Tyr is an indispensable L-amino acid because it is not supplied endogenously via Phe hydroxylation or only to a limited degree. L-tyrosine is important for the biosynthesis of the brain neurotransmitters (epinephrine, norepinephrine and dopamine), thyroxin and melanin skin pigments. Diurnal variations in blood Tyr concentrations are wide with Phe-free L-amino acid supplements that are supplemented with tyrosine. Fasting overnight blood Tyr concentrations are commonly low but then peak immediately following the intake of Phe-free Lamino acid supplements [114], even when given in equal frequent daytime doses. In $>80 \%$ of 12 PKU subjects, transiently higher than reference range Tyr concentrations occurred although they were not associated with adverse consequences [114, 297]. Therefore, in PKU, Tyr supplementation produces marked but unsustainable increases in plasma Tyr concentrations.

Tyr is added to all Phe-free L-amino acid supplements providing 9 to $11 \%$ of their L-amino acids. Therefore, most Phe-free L-amino acid supplements provide approximately $100 \mathrm{mg} / \mathrm{g}$ protein equivalent of Tyr which is almost double the concentration found in breast milk, and far exceeds the amount in a normal diet (in natural protein, in general $4 \%$ of $\mathrm{L}$-amino acids is from Tyr). A 
patient consuming $30 \mathrm{~g} /$ day protein equivalent from Phe-free L-amino acid supplements will take $3 \mathrm{~g} /$ day Tyr, and thereby exceeds the usual recommendations for the healthy population [202, 298]. The USA PKU guidelines suggest the following intake of Tyr: children under $1 \mathrm{y}, 1100-3000 \mathrm{mg} /$ day; $1-<4 \mathrm{y}, 2800-3500 \mathrm{mg} /$ day; and 4 y to adult 4000 to $6000 \mathrm{mg} /$ day [102], but it is unclear how this was calculated. The optimum amount of Tyr provided in a low Phe diet is unknown, but additional supplementation in excess of amounts added to Phe-free L-amino acid supplements is not associated with benefit.

To improve neuropsychological functioning, some clinics gave additional Tyr to the amounts added to Phefree L-amino acid supplements [299]. Meta-analysis of 3 randomized, cross-over, trials [300-302] studying 56 subjects given $2500 \mathrm{mg} /$ day [300] or $100 \mathrm{mg} / \mathrm{kg} /$ day [301, 302] of Tyr found that although there was improvement in blood Tyr concentrations, it was not associated with improvements in neurological outcome [303].

Finally, adding additional Tyr powder in a low Phe diet is problematic. Although it has a bland taste, it has a poor solubility leading to uncertainty about the actual amount received when added to liquids; the additional dose required is usually small and so it is difficult to measure with accuracy and administer evenly throughout the day.

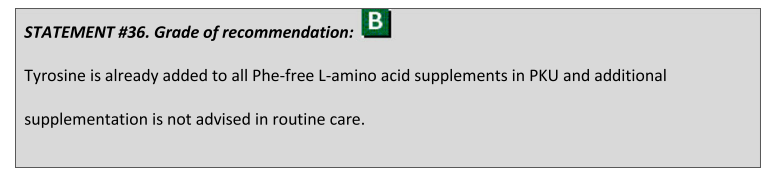

\section{Large neutral amino acids}

Non Phe LNAA include tryptophan, Tyr, histidine, methionine, threonine, leucine, isoleucine and valine. They are considered to have several potential functions in PKU: 1) lower blood Phe by competing with Phe uptake at the blood gut barrier [236-238] although this is not reported by all studies; 2) reducing brain Phe concentrations by providing competition with Phe to cross the blood-brain barrier [240, 242, 304, 305]; 3) increasing cerebral neurotransmitter concentrations (serotonin, norepinephrine, and epinephrine) [242]; and 4) increasing some cerebral large neutral amino acid concentrations such as Tyr, tryptophan, valine, leucine and isoleucine and BCAA [242]. However, in a randomised controlled trial of 16 patients, Schindeler et al. [235] examined the effect of LNAA in combination with diet and Phe-free L-amino acid supplements. They concluded that additional supplementation of LNAA was of limited value, but it may be of benefit in those unable to adhere to their Phe-free L-amino acid supplements [235].
Although some centres routinely administer Phe-free LNAA supplements to older patients who are unable to adhere to dietary treatment, these supplements remained untested in children under the age of 11 years. Their use is also not reported in pregnancy. Overall in PKU, there has been little evidence to support their routine use and further research is required to ascertain the ideal dosages and amount of each specific L-amino acid within the LNAA supplement.

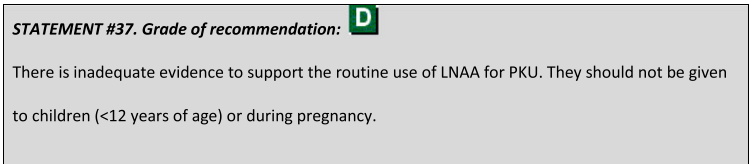

\section{IIIness}

It is well established that Phe levels increase during illness. Although patients are not at acute risk, Phe concentrations are likely to remain high until symptoms have abated. In addition, as shown with other IMD, it is known that children experience more illness episodes in the early years when, in PKU, they are particularly vulnerable to the effects of sustained high (and maybe also to fluctuating) Phe concentrations. In general, medical management of illness should be the same as for other children. However, some precautions are of importance as discussed in the next paragraph.

Infection, as occurs in all infants and children, affects their need for and utilization of energy and protein. According to Gardiner and Barbul [306], the ability of the small intestine to absorb L-amino acids is impaired during sepsis. Metabolic changes during infection include increased nitrogen loss, increased need for energy, catabolism of muscle protein leading to elevation of plasma Phe concentrations, conversion of L-amino acids to glucose, and decreased synthesis of acute phase proteins by the liver. Mild to moderate infection increases energy requirements by 20 to $30 \%$ [307]. Severe infection increases energy needs by approximately $50 \%$ above basal level [308] and it is estimated there is a $13 \%$ increase in energy expenditure per degree Celsius of fever [309]. Carbohydrate has been shown to improve nitrogen balance more than the isocaloric amount of fat in catabolic patients on parenteral nutrition [310]. In addition, there is evidence that Phe-free L-amino acid supplements suppress blood Phe concentrations [234]. Therefore, we consider it important that Phe-free L-amino acid supplements and high carbohydrate drinks are administered during infection to help decrease muscle protein loss and potentally lessen impact on deteriorating blood Phe control, although this remains unstudied. The importance of lowering natural protein during each illness episode is also unclear, although lowering natural protein during illness episodes may be 
necessary in patients treated by diet and/or BH4 [311]. Antipyretics should be administered for high temperatures. Treatment with antipyretics/analgesics like paracetamol and ibuprofen should be considered to improve food, fluid and energy intake during illness. Table 6 summarizes dietary advice for illness.

For any illness, if suitable aspartame-free medication cannot be sourced, it is better to use aspartamecontaining medications (for example antibiotics) rather than leave a child without treatment. Special attention should be given to ensure routine vaccination according to international/national standards. Gastroenteritis leads to a catabolic status. In young infants, oral vaccination against rotavirus is possible and recommended in most countries.

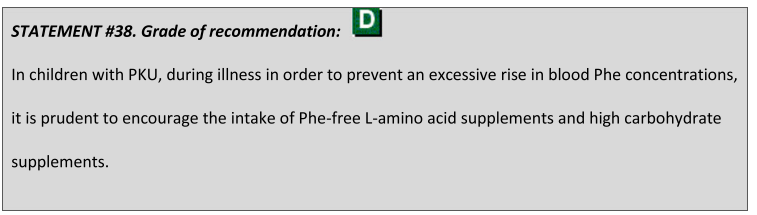

\section{Parenteral nutrition}

There is very limited information about the management and outcome of patients with PKU requiring treatment with parenteral nutrition. Infants, particularly those who are premature, and young children requiring long-term parenteral nutrition, are likely to be at risk from permanent damage if blood Phe concentrations cannot be controlled. Single case studies of premature infants with PKU given standard preparations of amino acid intravenous solutions for limited periods have had very high blood Phe levels although apparently without adverse effects on long-term neurological outcome [312-314]. A specially prepared Phe-free intravenous amino acid

Table 6 Dietary advice for illness in PKU

\begin{tabular}{|c|c|}
\hline Diet & Dietary advice \\
\hline $\begin{array}{l}\text { Phe-free L-amino acid } \\
\text { supplement }\end{array}$ & $\begin{array}{l}\text { Maintenance of Phe-free L-amino acid } \\
\text { supplement intake to support protein } \\
\text { synthesis. } \\
\text { It is better to give smaller, frequent doses } \\
\text { throughout the day. }\end{array}$ \\
\hline High carbohydrate intake & $\begin{array}{l}\text { Encourage frequent high carbohydrate } \\
\text { supplements, e.g. glucose polymer solution. }\end{array}$ \\
\hline Natural protein intake & $\begin{array}{l}\text { In practice, a reduced appetite leads to a } \\
\text { lower natural protein intake. }\end{array}$ \\
\hline Medications & $\begin{array}{l}\text { All treatment specific medication should be } \\
\text { continued during illness. Continue } \mathrm{BH} 4 \text { if } \\
\text { already prescribed. } \\
\text { Medications should be free of aspartame } \\
\text { in PKU. }\end{array}$ \\
\hline Treat precipitating factors & $\begin{array}{l}\text { e.g. anti-pyretics for pyrexia, antibiotics } \\
\text { (aspartame-free) for bacterial infections. }\end{array}$ \\
\hline
\end{tabular}

solution has been used in a 6 year old with PKU with an intra-abdominal malignancy, which effectively prevented high Phe levels [315]. A commercially available preparation with a lower content of branched chain amino acids designed for use in hepatic failure was used in a premature infant with PKU as this preparation also contained less than usual Phe [312] and in a child with a facial tumour [316]. However elevated Phe levels could not be completly prevented in these cases.

\section{Support}

Living with a life-long severe dietary restriction may adversely affect eating attitudes and behaviours and increase susceptibility to the development of eating disturbances [317]. Coping with and adhering to dietary treatment has been described as a stressor to both the patient and the family. Feeding behaviour problems are more common in young children with PKU $[115,243]$ and appear associated with the management of feeding behaviour rather than intrinsic to the condition. Food neophobia is also more prevalent in children with PKU, with children being particular about their food choices and untrusting of new foods when compared with control children without PKU [251]. However, early intervention, working alongside psychologists and play therapists, can play an important role in improving feeding behaviors and family mealtime interactions. Strategies that are used for general feeding problems apply to children with PKU e.g. positive caregiver role modelling, gradually increasing familiarity with new foods, consistent mealtime routines with adequate time allocation for eating.

It is commonly reported that children may need constant coercion to take their Phe-free L-amino acid supplements which is exhausting for caregivers and some may resort to strategies such as yelling, grounding or taking away privileges [247]. A study of feeding problems in young children indicated that almost $50 \%$ had difficulty with its administration and all children had been given Phe-free L-amino acid supplements since early infancy [243]. Some of these problems may have been related to the developmental age of children or consistency of approach by caregivers. Overall, there is lack of research on the best strategies to support caregivers in the maintenance of administration of Phe-free L-amino acid supplements.

In adolescents and adults with PKU the occurrence of eating disorders has not been systematically explored and rarely reported so may be undetected and untreated. This is an area that requires further study in PKU. Regular health professional support, especially from a psychologist, may provide some measure of protection. 


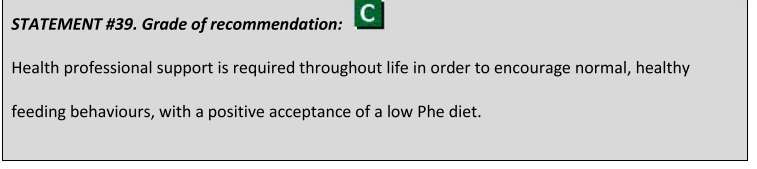

\section{Treatment in specific patient groups Maternal PKU}

PKU treatment aims to prevent maternal PKU syndrome [318]. High blood Phe levels during pregnancy have a teratogenic effect on the developing foetus that can result in growth retardation, microcephaly, intellectual disabilities and birth defects, including congenital heart defects (CHD) $[318,319]$. When treatment recommendations are adhered to, the chances of a good outcome are comparable to the normal population.

\section{Risk of CHD}

Lenke and Levy [318] reported CHD in 27 infants of mothers with assigned blood Phe levels (APL) of $>1200 \mu \mathrm{mol} / \mathrm{l}$ and in 7 infants when blood Phe levels were between 900 and $1200 \mu \mathrm{mol} / \mathrm{l}$. These APL were selected as the highest of 2 or 3 plasma Phe levels [318]. In the Maternal PKU Collaborative Study (MPKUCS), Levy et al. [320] described 34 offspring with CHD from pregnancies in women with PKU and in 1 offspring from a mother with MHP. All PKU mothers had APL $>900 \mu \mathrm{mol} / \mathrm{l}$ and did not achieve metabolic control before the 8th week of gestation [320]. In a further report from the MPKUCS, Platt et al. [321] described 31 offspring with CHD. When maternal blood Phe levels were 120-360 $\mu \mathrm{mol} / \mathrm{l}$ during the first 8 weeks of gestation, no cases of CHD were described; when Phe levels were 360 $600 \mu \mathrm{mol} / \mathrm{l}$, there was one case of CHD; when Phe levels were $600-900 \mu \mathrm{mol} / \mathrm{l}$, there were 5 cases of $\mathrm{CHD}$; and when Phe levels were $>900 \mu \mathrm{mol} / \mathrm{l}$, there were 26 cases of CHD [321]. Table 7 provides the percentages of offspring with CHD.

In untreated or non-optimally treated pregnancies, increased frequencies of intrauterine growth retardation (IUGR), intellectual disability, microcephaly [318, 319, 322] and other congenital abnormalities have been described in the MPKUCS [323] and case reports (Table 8).

\section{No treatment}

For PKU women of childbearing age, no treatment is necessary when untreated blood Phe levels are $<360 \mu \mathrm{mol} / \mathrm{l}$. Levy et al. [324] showed that mean untreated blood Phe levels $<400 \mu \mathrm{mol} / \mathrm{l}$ had no effect on birth measurements and no effect on offspring IQ. Platt et al. [321] confirmed no increased rate of birth defects in (un)treated blood Phe levels between 120 and $360 \mu \mathrm{mol} / \mathrm{l}$. Levy et al. [325] even demonstrated that there was no correlation between the offspring's IQ and mothers untreated APL $<600 \mu \mathrm{mol} / \mathrm{l}$. Waisbren et al. [326] reported that the offspring of women with untreated MHP had cognitive and behavioural development similar to control subjects.

\section{Treatment goals/target Phe levels}

Woman with PKU should start a Phe-restricted diet before conception. Many features of the maternal PKU syndrome are preventable by starting a low Phe diet before conception or early in pregnancy [319, 320, 327-329]. Children born to mothers with PKU who attain satisfactory blood Phe control before or very early in pregnancy appear to begin life with normal potential. Maternal delay in attainment of acceptable blood Phe control is associated with decline in offspring developmental outcome/IQ scores [326, 329, 330].

In prospective studies, there is no effect on infant birth measurements and final IQ when mother's mean blood Phe levels are $<360 \mu \mathrm{mol} / \mathrm{l}$. The MPKUCS study indicated that the major factors associated with good child outcome was normal maternal intelligence and well-treated pregnancies with blood Phe control between 120 and $360 \mu \mathrm{mol} / \mathrm{l}$ [319, 322, 326, 329]. Widaman [51] demonstrated a threshold effect of a mother's average blood Phe value of $400 \mu \mathrm{mol} / \mathrm{l}$ in relationship to offspring's IQ. With every further increase of $60 \mu \mathrm{mol} / \mathrm{l}$ Phe, the IQ drops by 4.7 points [51]. In addition it seems to be important that blood Phe concentrations are maintained consistently even within target range [330]. The prevention of CHD requires initiation of the low Phe diet before conception or early in pregnancy ( $<8$ th week) [320]. Dietary management that is too strict may be associated with a risk of IUGR in the offspring as described by Teissier et al. [331]. As IUGR is related to an increased risk of diabetes, cardiovascular disease and hypertension later in life, Phe levels below $120 \mu \mathrm{mol} / \mathrm{l}$ should be avoided.

Table 7 Percentages of offspring with congenital heart disease with Maternal Off-Diet Phe Levels $(\mu \mathrm{mol} / \mathrm{L})$

\begin{tabular}{llllll}
\hline & $\geq 1200 \mu \mathrm{mol} / \mathrm{I}$ & $900-1200 \mu \mathrm{mol} / \mathrm{l}$ & $600-900 \mu \mathrm{mol} / \mathrm{l}$ & $180-600 \mu \mathrm{mol} / \mathrm{l}$ & Control group/normal population \\
\hline Lenke and Levy 1980 [318] & $12 \%$ of $n=225$ & $15 \%$ of $n=46$ & $6 \%$ of $n=33$ & $0 \%$ of $n=44$ & $0.8 \%$ in normal population \\
MPKUCS Koch 2003 [319] & $11 \%$ of $n=257$ & $5 \%$ & $3 \%$ & $2 \%$ of $n=66$ & $1 \%$ CHD of $n=100$ control pregnancies \\
& & $n=91$ with 600-1200 $\mu \mathrm{mol} / \mathrm{l}$ & & \\
\hline
\end{tabular}


Table 8 Reported malformations in the literature

\begin{tabular}{l} 
Malformations \\
\hline Congenital heart defects \\
- Tetralogy of Fallot \\
- ventricular septal defect \\
- mitral/aortic stenosis \\
- patent ductus arteriosus \\
Dysmorphology \\
- microcephaly \\
- coloboma \\
- malformed eyelid, ptosis \\
- hypertelorism \\
- cleft palate \\
- malformed ears \\
- simian creases \\
- fused digits \\
- widely spaced toes \\
Other: \\
- anencephaly \\
- oesophageal atresia \\
- renal agenesis, Potter syndrome \\
- hypospadias \\
- hydrocele \\
- anal fistula, anal atresia
\end{tabular}

Reported in the MPKUCS [323] and case reports

As women with untreated Phe levels between 360 and $600 \mu \mathrm{mol} / \mathrm{l}$ need to return to dietary treatment prior to conception, some may consider that during child bearing years, women should continue a small dose of Phe-free L-amino acid supplements to help retain acceptance of its taste, but this practice remains unproven.

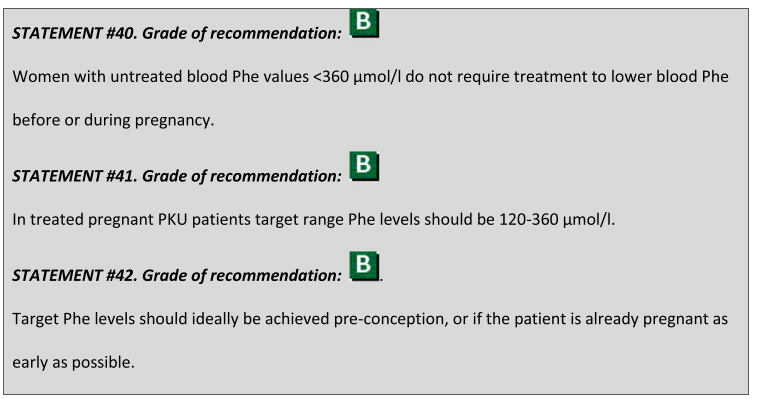

For optimal pre-conception treatment, stable blood Phe control within target range should be maintained before PKU women conceive. The time to reach stable and acceptable blood Phe concentrations varies between women. It is influenced by personal conditions (organizational skills, IQ, work conditions) and family support, which will affect the ability to adhere to strict long-term diet. In general women planning pregnancy are well motivated. The timing of medical consent to stop contraceptive strategies varies between centres and countries. In some European centres, women with blood Phe levels within target range for as little as 2 weeks may be advised to stop contraceptives, other centres may advise several months. We recommend contraceptive strategies should only be discontinued after stable Phe levels within target range have been achieved for a minimum of 2 weeks. There is no evidence that maintaining blood Phe levels within target range for a period of 2-3 months vs 2 weeks pre-conception is associated with better outcome. Shorter pre-conception periods may help maintain patient motivation. However, achieving 'blood Phe levels within target for at least 2 weeks' may take several weeks as some women with PKU need time to adapt to the rigorous demands of a low Phe diet and obtain regular and consistent access to special dietary products.

\section{Pregnancy planning and medical follow-up}

Maternal PKU is considered a high risk pregnancy as it is difficult to prevent high or low blood Phe concentrations, necessitating follow-up by a obstetrician well informed about PKU as well as a metabolic dietician and metabolic physician. Minimal outpatient clinic visits of once during each trimester is recommended, but many health practitioners may advocate more frequent followup and the intensity of monitoring will depend on individual needs and metabolic control.

Metabolic control is based on weekly Phe blood spots pre-conception and at least twice weekly during pregnancy, with speedy laboratory turnaround times.

PKU women should undertake the same pre-conception screening as recommended for healthy women and should receive education about healthy lifestyle and behaviour. These recommendations are found in the (inter)national maternity guidelines [332].

As maternal PKU treatment needs considerable effort from the woman and her partner, failure to conceive requires special attention. Therefore, it is reasonable to refer well-controlled patients to a fertility specialist earlier than after 1 year (the WHO criteria of sterility) and it seems appropriate to recommend a time period of 6 months [333].

In maternal PKU there are 2 main risks for fetal development: growth retardation and birth defects including CHD. Therefore, detailed follow-up by ultrasound examination specific for high risk pregnancies (especially for inadequate metabolic control) is highly recommended from the very early beginning of pregnancy with screening for organ development at 18-22 weeks of pregnancy [320, 334].

Post delivery women with PKU should receive routine obstetric care and should be encouraged to return to standard dietary or pharmacological treatment [335]. There is a relationship between postnatal child stimulation in the home environment and their developmental outcomes [326]. There are no studies available about Phe toxicity of the mother post-delivery and the risk of postnatal depression. 


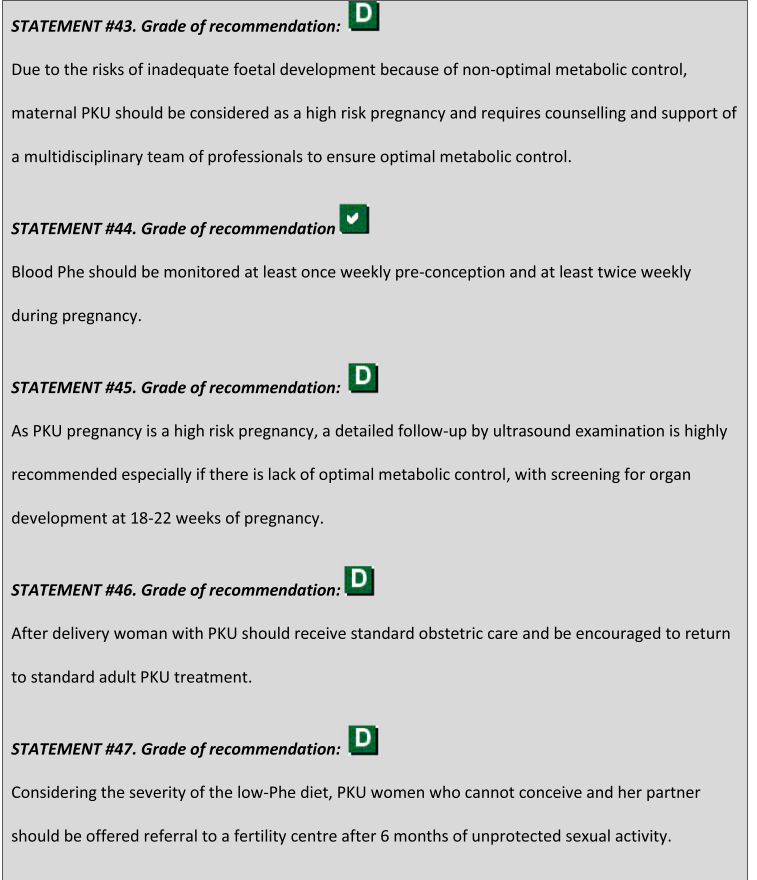

Offspring follow up after delivery Infants suffering from a birth defect or severe health problems must be treated after birth. An echocardiogram should be considered in all infants who are conceived by women with high blood Phe levels and in those with poor maternal blood Phe control during pregnancy [333]. After sub-optimal pregnancy treatment, infants commonly have smaller birth measurements and delayed cognitive development. Children should be followed up, preferably in specialized centres, similarly to other 'at risk' infants e.g. preterm infants or small for gestational aged children.

\section{Prevention of unplanned pregnancies}

Unplanned pregnancies in woman with PKU are a significant health problem [336]. In 2008 in Europe, in the general population $44 \%$ of conceptions were unplanned [337]. Prevention of the maternal PKU syndrome requires ongoing education from childhood into adulthood about foetal risks associated with high plasma Phe concentrations and importance of pregnancy planning $[338,339]$. This information should be re-inforced by all members of the core PKU team. Patients with PKU also consider that counselling and education on the dangers of unplanned pregnancy is required [11]. For women with untreated Phe levels $<600 \mu \mathrm{mol} / \mathrm{l}$ who may have stopped diet in adolescence, a robust transition process between paediatric and adult services is essential to ensure they are not lost to follow-up. Clinic patient registries and on-going contact with the PKU team are important.

PKU women at childbearing age, with all forms of HPA, should receive detailed counselling regarding family planning and the risks of adverse foetal effects as a consequence of elevated Phe levels. If teenage PKU girls and women are suspected of sexual activity, the most effective birth control methods should be advised. In 60 PKU women, factors associated with contraception usage were the extent to which women felt socially supported to use contraception $(r=0.64)$ along with positive attitudes about birth control $(r=0.66)$ and knowledge of family planning $(r=0.43)$ [340].

From the age of 12 years (beginning of puberty), all patients should receive systematic age-related sex education, with professional counselling about the risk of unprotected sexual contacts. They should be informed that unplanned pregnancy can occur even during the first menstrual cycle. At least one PKU team member should be able to provide sex education information. Pre-conception education initiatives that have been developed in for example diabetes could be adapted for PKU care [341, 342].

It is important that all advice is individually tailored, particularly for women with psychological and/or intellectual impairment. Individual cultural and religious background should be considered in patient counselling.

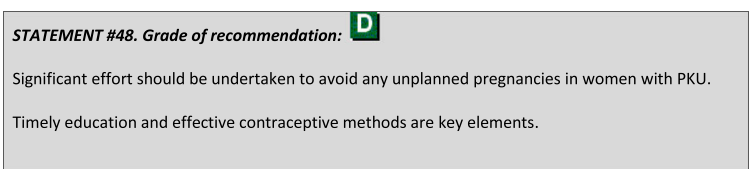

\section{Nutritional recommendation in maternal PKU}

The nutritional status of women with PKU both during pre-conception and pregnancy is likely to have significant influence on foetal and infant outcomes. Attentive and proactive management is essential. Maternal weight loss, over restriction of Phe intake, lack of folic acid, and vitamin B12 may all affect foetal outcome. Practical education of women giving them the skills and knowledge to manage their dietary treatment is a key component of care.

Phe tolerance Maternal Phe tolerance is influenced by the severity of PKU but will vary even between pregnancies in the same patient according to adherence with Phe-free L- amino acid supplements, adequacy of energy intake and changes in weight, trimester of pregnancy, and existence of foetal PKU. Reported Phe tolerance is given in Table 9. If the foetus has PKU, it has been reported that Phe tolerance barely increases in the second and third trimester of pregnancy [343]; thereby a low 
Table 9 Reported Phe tolerance during pregnancy in maternal PKU

\begin{tabular}{lllll}
\hline Reference & Number of patients & 1st trimester & 2nd trimester & 3rd trimester \\
\hline Vockley et al. 2014 [102] & 0 (USA guidelines) & $265-770 \mathrm{mg} /$ day & $400-1650 \mathrm{mg} /$ day & $700-2275 \mathrm{mg} /$ day \\
Acosta et al. 2001 [350] & 240 & $456 \pm 233$ to $684 \pm 413 \mathrm{mg} / \mathrm{day}$ & $528 \pm 269$ to $528 \pm 269 \mathrm{mg} / \mathrm{day}$ & $938 \pm 542$ to $1248 \pm 513 \mathrm{mg} / \mathrm{day}$ \\
Thompson et al. 1991 [351] & 1 & $6 \mathrm{mg} / \mathrm{kg}$ bodyweight/day & & $30 \mathrm{mg} / \mathrm{kg}$ bodyweight/day \\
Kohlschutter et al. 2009 [343] & 3 & $400 \mathrm{mg} /$ day & & $1700 \mathrm{mg} /$ day (non-foetal PKU) \\
& & & & maximum 600 mg/day (foetal PKU) \\
Duran et al. 1999 [232] & 5 & $250-500 \mathrm{mg} /$ day & $300-500 \mathrm{mg} /$ day & $1300-1500 \mathrm{mg} /$ day \\
Rohr et al. 1987 [369] & 3 & $450-800 \mathrm{mg} /$ day & $720-1300 \mathrm{mg} /$ day & \\
\hline
\end{tabular}

Phe tolerance in the third trimester of pregnancy may indicate foetal PKU [343].

From the second trimester onwards a period of rapidly increasing Phe requirement begins owing to foetalmaternal anabolism. As Phe is an essential L-amino acid, it is important that dietary Phe intake is increased (by 50 to $100 \mathrm{mg} /$ day) without delay if blood Phe concentrations are $\leq 120 \mu \mathrm{mol} / \mathrm{L}$. Slow increases in natural protein intake may prolong the length of time blood Phe concentrations are $<120 \mu \mathrm{mol} / \mathrm{l}$. Teissier et al. reported from 155 pregnancies in 86 PKU women that Phe intakes were lower in a group with IUGR from the fifth to the 8 month of pregnancy. The longer the duration of time blood Phe below $120 \mu \mathrm{mol} / \mathrm{l}$, the higher the risk of IUGR [331].

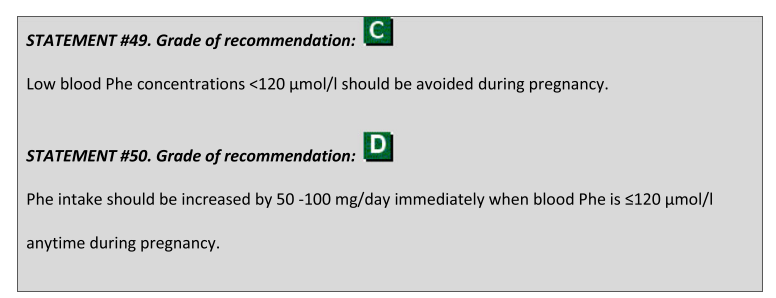

Weight gain and energy requirements Low energy intake, accompanied by weight loss is common, particularly in the first trimester of pregnancy in PKU and this is associated with higher blood Phe concentrations [344]. Inadequate energy intake may be due to dislike of low protein foods, poor adherence with lack of Phefree L-amino acid supplements, limited availability of low protein foods, inability to prepare low protein meals or poor appetite associated with nausea and vomiting. Poor maternal weight gain (less than $70 \%$ of recommended) and foetal microcephaly are correlated [345]. Microcephaly significantly decreases when maternal weight gain is adequate [345]. There is also evidence that inadequate gestational weight gain is associated with decreased foetal growth and birth weight both in the general population [346] and in PKU [347, 348], although further study is required. Data from a French survey reported in 135 pregnancies that the BMI of mothers was lower than the general population but there was no direct correlation with IUGR in children [349]. In a single adult centre in London, women with PKU lost weight in the first trimester and had a weight gain below that recommended for pregnancy [330], probably reflecting a low energy intake.

In the MPKUCS, maternal energy intake was significantly and negatively correlated with plasma Phe concentrations during the last 2 trimesters of pregnancy [350]. In addition, case studies have described difficulty in maintaining blood Phe control due to weight loss and low energy intake in the first trimester [347, 351].

Energy requirements vary considerably for individuals but they should be tailored to pre-pregnancy BMI, rate of weight gain, maternal age, gestational stage of pregnancy, physical activity levels and blood Phe control. Any additional energy costs associated with the maintenance of a normal pregnancy are due to greater maternal and foetal-placental tissue mass, increased energy expenditure attributable to increased basal metabolism and changes in the energy cost of physical activity [352, 353]. Published guidelines for normal maternal energy requirements do vary between 'expert' groups and are given in Table 10. Most dietary studies in well-nourished women in non-PKU pregnancy have shown no or only minor increases in energy intake that only partially cover the estimated energy cost of pregnancy [353]. The best way to determine if energy needs are being met is to carefully monitor maternal weight change in pregnancy.

Birth weights between 3.1 and $3.6 \mathrm{~kg}$ (mean, $3.3 \mathrm{~kg}$ ) are associated with the optimal ratio of maternal and foetal health outcomes [354]. This is in turn associated with a pregnancy weight gain of 10-14 kg (mean $12 \mathrm{~kg}$ ) [354].

If there is maternal weight loss, additional energy from low protein foods (e.g. pasta, bread) and energy supplements (glucose polymer/fat emulsions) should be considered, and weight monitored weekly until weight gain is satisfactory [355]. However, excess weight gain should also be prevented as maternal obesity is associated with additional complications throughout pregnancy and increased health risks to the mother and her infant 
Table 10 Additional energy requirements in general population (non-PKU) pregnancy

\begin{tabular}{|c|c|c|c|}
\hline \multirow[t]{2}{*}{ References } & \multicolumn{3}{|c|}{ Additional energy requirements in pregnancy (kcal/day) } \\
\hline & 1st trimester & 2nd trimester & 3rd trimester \\
\hline UK SACN (2011) [353] & None & None & $191 \mathrm{kcal} / \mathrm{day}$ \\
\hline FAO/WHO/UNU (2001) (based on gestational weight gain of $12 \mathrm{~kg}$ ) [393] & $85 \mathrm{kcal} /$ day & $360 \mathrm{kcal} / \mathrm{day}$ & $475 \mathrm{kcal} /$ day \\
\hline $\begin{array}{l}\text { aOM dietary reference intakes (2005) [382] } \\
\text { For women 19-50 years }\end{array}$ & None & $340 \mathrm{kcal} /$ day & $452 \mathrm{kcal} / \mathrm{day}$ \\
\hline $\begin{array}{l}\text { aOM dietary reference intakes (2005) [382] } \\
\text { for girls 14-18 years }\end{array}$ & None & $340 \mathrm{kcal} /$ day & $452 \mathrm{kcal} /$ day \\
\hline
\end{tabular}

${ }^{a}$ Energy requirements based on following assumptions: total energy expenditure changes little and weight gain is small during the first trimester so additional energy recommended during second and third trimester only

[356, 357]. Overall, in maternal PKU weight gain should be similar to the healthy population (Table 11) with emphasis on avoiding weight loss particularly in the first trimester of pregnancy.

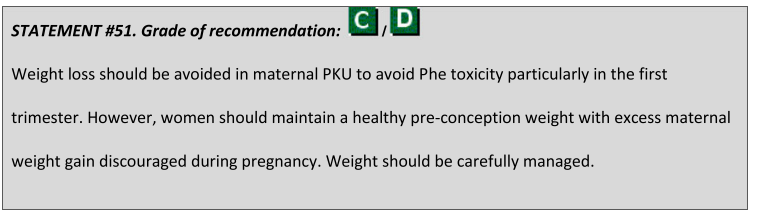

Protein requirement Additional protein requirement during pregnancy is due to newly deposited protein and the maintenance costs associated with increased body weight [202]. The recommended additional protein intake recommended by FAO/WHO/UNU [202] in nonPKU pregnancy is $1 \mathrm{~g}$ /day during the first trimester, $10 \mathrm{~g} /$ day during the second trimester and $31 \mathrm{~g} /$ day during the third trimester, with an efficiency for protein utilization estimated to be $42 \%$. In maternal PKU, total protein requirements are not accurately defined and can only be extrapolated from non-PKU maternal requirements. Reports of protein prescription from Phe-free L-amino acid supplements (with or without natural

Table 11 The Institute of medicine (USA) and National Research Council (USA) Committee guidelines for pregnancy weight gain in the general population [488]

\begin{tabular}{|c|c|c|}
\hline \multirow[t]{2}{*}{ Pre-pregnancy BMI } & \multirow[t]{2}{*}{$\begin{array}{l}\text { Total weight } \\
\text { gain } \mathrm{kg}\end{array}$} & \multirow{2}{*}{$\begin{array}{l}\text { Rates of Weight } \\
\text { Gain }{ }^{+} \text {2nd and } \\
\text { 3rd Trimester kg } \\
\text { Mean (range) in } \\
\text { kg/week }\end{array}$} \\
\hline & & \\
\hline Underweight $\left(<18.5 \mathrm{~kg} / \mathrm{m}^{2}\right)$ & $12.5-18$ & $0.51(0.44-0.58)$ \\
\hline Normal weight (18.5-24.9kg/m²) & $11.5-16 \mathrm{~kg}$ & $0.42(0.35-0.50)$ \\
\hline Overweight (25.0-29.9 kg/m²) & $7-11.5 \mathrm{~kg}$ & $0.28(0.23-0.33)$ \\
\hline Obese $\left(\geq 30.0 \mathrm{~kg} / \mathrm{m}^{2}\right)$ & $5-9 \mathrm{~kg}$ & $0.22(0.17-0.27)$ \\
\hline
\end{tabular}

${ }^{\mathrm{a} B M I}$ body mass index, Adolescents should aim for weight gains at upper end of recommendations. ${ }^{+}$Calculations assume a $0.5-2 \mathrm{~kg}$ weight gain in the first trimester protein) have varied widely: reference nutrient intake $(\mathrm{RNI})+15 \%[355], \geq 70 \mathrm{~g} /$ day total protein [102], and $100 \mathrm{~g} /$ day amino acids [358]. There are no reported studies examining the utilisation of Phe-free L-amino acid supplements in specifically maternal PKU.

However, it is established that the delivery of the prescribed amount of Phe-free L-amino acid supplements is important during pregnancy. In the MPKUCS study, lower total protein intakes $(<\mathrm{USA} \mathrm{RNI})$ were associated with worse blood Phe control [348]. In another report, mothers who had an inadequate protein intake (less than $50 \%$ of recommended amounts) in the first trimester mainly due to nausea and vomiting, together with poor metabolic control, had a higher risk of CHD compared to mothers who had an adequate protein intake and similar blood Phe concentrations [345, 359]. Lower maternal total protein intakes were negatively associated with birth weight and length of the newborns [348]. Lower concentrations of the amino acids: proline, valine, methionine, isoleucine, lysine and arginine in PKU mothers have also been linked with low intake of Phe-free L-amino acid supplements and a higher risk of CHD in their offspring [360]. Inadequate intake of micronutrient supplemented Phe-free L-amino supplement has been associated with low intakes of vitamin B12 and this is also associated with an increased risk of CHD [359].

Duran et al. demonstrated the impact of good adherence with Phe-free L-amino acid supplements on lowering blood Phe concentrations in pregnant women [232].

We have adopted the recommendation that in maternal PKU, a total protein intake of $\geq 70 \mathrm{~g} /$ day is required, but it is also important to consider individual patient weight and the additional protein requirements of each pregnancy trimester. In maternal PKU, more data is required about prescribed and actual total protein intakes compared with infant outcome measures. The protein substitutes based on LNAA [361] and glycomacropeptide have not been reported during pregnancy and are thereby not recommended for maternal PKU until their safe use is established. 


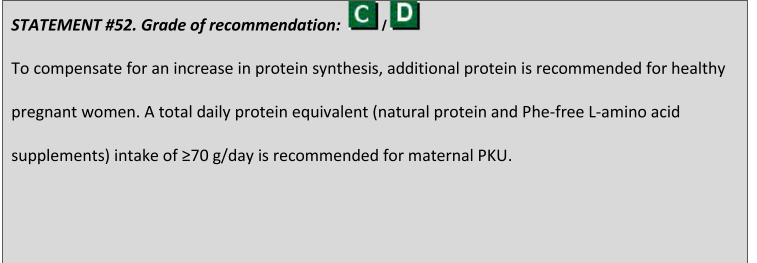

Tyrosine supplementation Many PKU centres advocate additional Tyr supplementation at some stage during pregnancy [333, 347, 351,362-371], although the specific requirement in maternal PKU is unknown. The amount of Tyr supplementation given is highly variable and usually without justification for the dose chosen (Table 12). Normally, Tyr is a non-essential amino acid synthesized from Phe. In PKU, Tyr cannot be synthesized from Phe, resulting in lower blood Tyr concentrations. It has been proposed that some of the birth defects seen in maternal PKU may be related to low Tyr concentrations [372], and that Tyr supplementation during pregnancy may help improve foetal outcome [370].

In non-maternal PKU, it has been established that Tyr supplementation raises blood Tyr [303]. In maternal PKU, Tyr supplementation raises maternal Tyr concentrations above the recommended minimum concentration $(>45 \mu \mathrm{mol} / \mathrm{l})$ for a period of $\geq 3 \mathrm{~h}$ and is associated with a markedly increased ratio of Tyr to LNAA [370]. However, Tyr supplementation leads to variation in blood Tyr between fasting and fed state, and Tyr concentrations $>200 \mu \mathrm{mol} / \mathrm{L}$ have been observed at some time points within a 24-h period [297]. It is also suggested that foetal Tyr concentrations in blood will be some 1.8 to 3.3 times higher than in the maternal blood [297, 373]. A toxic effect of a combination of mildly increased Phe and Tyr was demonstrated experimentally in rats [374].

Table 12 Reported Tyr supplementation during pregnancy in maternal PKU

\begin{tabular}{|c|c|}
\hline Author & Tyr supplementation \\
\hline Coutts 1979 [358] & $>10 \mathrm{~g} /$ day \\
\hline $\begin{array}{l}\text { Singh et al. } 2014 \text { [361], } \\
\text { Vockley et al. } 2014 \text { [102] }\end{array}$ & 6000 to $7600 \mathrm{mg} /$ day \\
\hline Rohr et al. 1987 [369] & $\begin{array}{l}\text { Up to } 6 \mathrm{~g} / \mathrm{d}(\mathrm{did} \text { not } \uparrow \text { blood } \\
\text { Tyr }>30 \mu \mathrm{mol} / \mathrm{l})\end{array}$ \\
\hline Davidson et al. 1989 [364] & $6.4-11.9$ g/day \\
\hline Brenton et al. 1996 [363] & 2 g/day \\
\hline Maillot et al. 2007 [333] & $\begin{array}{l}8 \mathrm{~g} / \text { day (total from Phe-free } \mathrm{L} \text {-amino } \\
\text { acid supplements and Tyr supplements) }\end{array}$ \\
\hline Thompson et al. 1991 [351] & $\begin{array}{l}160 \mathrm{mg} / \mathrm{kg} / \text { day- starting point and } \\
\text { then titrate according to Tyr levels }\end{array}$ \\
\hline
\end{tabular}

The FAO/WHO/UNU 2007 suggests the aromatic amino acids (Phe/Tyr combined) requirements for healthy adults are only $30 \mathrm{mg} / \mathrm{g}$ protein [202]. Therefore, women taking $75 \mathrm{~g}$ /day total protein would require $2250 \mathrm{mg}$ Tyr daily. All Phe-free L-amino acid supplements contain Tyr. The amounts of Tyr in $60 \mathrm{~g} /$ day protein equivalent from Phe-free L-amino acid supplements varies between 5 and $7 \mathrm{~g} /$ daily and is well in excess of daily requirements. Thereby, this would suggest that any additional Tyr to what is already provided by the L-amino acid supplements is unnecessary. However, in PKU due to aberrant Phe/Tyr metabolism, it may be inappropriate to compare free Tyr requirements to either the $\mathrm{FAO} / \mathrm{WHO} / \mathrm{UNU} 2007$ aromatic amino acid or protein requirements.

Furthermore, the MPKUCS study found no relationship between infant outcomes and maternal blood Tyr concentrations before and during pregnancy [319]. An optimal range for plasma Tyr concentration in maternal PKU has not been established. It is important that excessive Tyr supplementation should be avoided as the safety of Tyr supplementation during pregnancy has not been extensively studied.

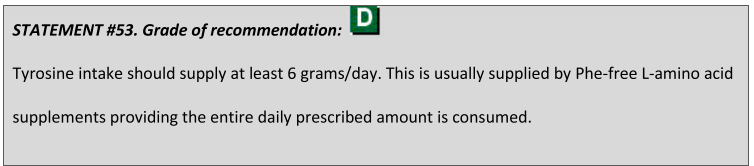

Emergency diet during unplanned pregnancies Even in women with an unplanned pregnancy and high blood Phe concentrations it is essential to reduce Phe intake, although this should not be accompanied with weight loss so avoiding catabolism. Women who become pregnant without appropriate blood Phe control will need significant support to attain Phe levels within the recommended target range in a timely fashion (Dietary education programmes for maternal PKU section). Women should be given emergency supplies of Phe-free L-amino acid supplements and low protein foods until their own supply can be established through the health or insurance systems. Baseline blood Phe concentrations, anthropometry, nutritional biochemistry should be established. The teaching of home blood Phe sampling is necessary.

As a starting point, the initial Phe allocation should be the same as the Phe tolerance when aged between 1 and 5 years of age and will depend on the severity of PKU. In the absence of this information, a guideline for women's predicted Phe tolerance at the start of pregnancy is suggested by Maillot et al., (Table 13) and Phe intake should then be adjusted according to blood Phe levels [333].

Data show that uncontrolled maternal blood Phe levels (over $600 \mu \mathrm{mol} / \mathrm{l}$ ) over 10 weeks of pregnancy result in a high risk of a child developing severe clinical disabilities 
Table 13 Predicted amount of Phe tolerated during early pregnancy according to blood Phe concentration on an unrestricted diet (modified from Maillot et al. 2007 [333])

\begin{tabular}{ll}
\hline $\begin{array}{l}\text { Blood Phe concentration } \\
(\mu \mathrm{mol} / \mathrm{L})\end{array}$ & $\begin{array}{l}\text { Initial amount of daily Phe to give at } \\
\text { the start of dietary treatment } \mathrm{mg} / \text { day }\end{array}$ \\
\hline$>2000$ & 150 \\
$1600-2000$ & 200 \\
$1200-1600$ & 300 \\
$1000-1200$ & 300 \\
\hline
\end{tabular}

(Tables 7,8$)$. Although the risks of CHD are within the first 8 weeks of pregnancy, the risk of impaired foetal development in general and especially to the brain is ongoing throughout pregnancy. Therefore, in such cases, non-directive counselling should be given, with the clinician informing about the high risk (15\%) of birth defects (minor or severe). A foetal heart ultrasound is recommended at 18-22 weeks gestation.

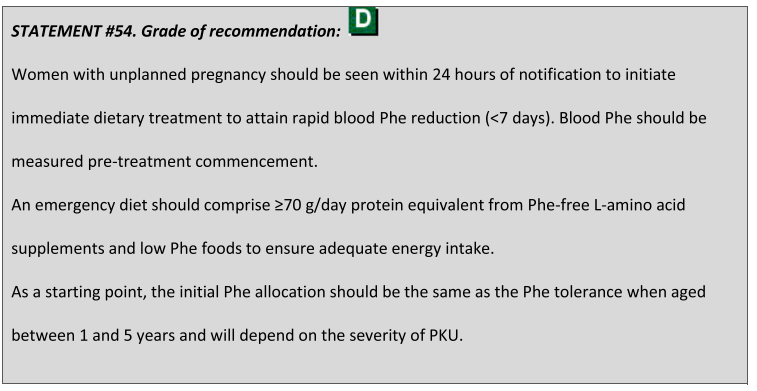

Nausea and vomiting/hyperemesis Nausea and vomiting affects up to $85 \%$ of all pregnancies [375]. This may affect the dietary intake and weight gain so should be aggressively treated. In PKU this can lead to elevated blood Phe levels. Symptoms generally begin around week 5 of gestation and typically stop by week 12 . Up to $15 \%$ of pregnant women experience persistent symptoms until delivery. Hyperemesis gravidarum (affecting between
$0.5-2 \%$ of women) represents the extreme end of the spectrum associated with dehydration and weight loss greater than $5 \%$ of pre-pregnancy weight. Besides nasogastric tubes, gastrostomy tubes have been used in 'healthy' pregnant women with hyperemesis gravidarum but are associated with infections and surgical risks [376]. Gastrostomy tube placement is also an option that has been used to deliver Phe-free L-amino acid supplements to women who are unable to restart diet due to severe nausea or palatability issues [377].

The treatment should be tailored to the individual but should include similar but adapted advice to non-PKU pregnancy. Useful additional advice in maternal PKU can be found in Table 14 .

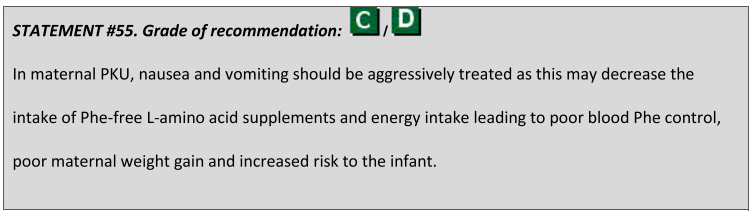

Folic acid supplementation Little attention has been paid to folic acid intake in maternal PKU. Folic acid is of critical importance both pre- and peri-conceptionally in protecting against neural tube defects in the developing foetus (first 28 days of pregnancy). There is now conclusive evidence from a number of randomized controlled trials that folic acid supplementation can prevent neural tube defects [378-380]. Folic acid requirements increase during pregnancy and many countries give an additional $400 \mu \mathrm{g} /$ day pre-conceptually and during the first 12 weeks of pregnancy (Table 15). The upper tolerable limit of folic acid in the healthy women recommended in Europe [381] and USA [382] is $1 \mathrm{mg}$ per day. However 'Diabetes UK' (www.diabetes.org.uk) recommends $5 \mathrm{mg} /$ day of folic acid in diabetes to prevent neural tube defects due to higher risk.

Whilst Phe-free L-amino acid supplements contain folic acid, the amount is variable and adherence with

Table 14 Advice to help nausea and vomiting in maternal PKU

Dietary and lifestyle Small frequent low protein meals and snacks that are high in carbohydrate (e.g. low protein toast, crackers) and low in fat to approaches avoid an empty stomach, feelings of hunger, and abdominal distension [375]. Cold meals may be better if nausea is associated with food smells. For women who have difficulty in eating solid foods, additional drinks of cold water supplemented with glucose polymer may be tolerated if sipped throughout the day. Women should avoid lying down immediately after meals.

Phe-free L-amino acid Give Phe-free L-amino acid supplements chilled and encourage up to 5 or 6 times during the day in small doses. The high supplements osmolality of Phe-free L-amino acid supplements may aggravate nauseas [347] and so may be better tolerated if given with extra fluid. If the smell of liquid or powdered Phe-free L-amino acid supplements is not tolerated, Phe-free L-amino acid tablets are worth consideration.

Any doses of Phe-free L-amino acid supplements lost through vomiting should be re-given. In extreme cases of vomiting and Phe-free L-amino acid supplements intolerance, hospital admission and administration of Phe-free L-amino acid supplement via a nasogastric tube could be considered.

Medication Safe antiemetic therapy and acid reducing medications should be considered with persistent vomiting and symptoms of dyspepsia and indigestion. 
Table 15 National folic acid requirements in general population (non-PKU) pregnancy [489]

\begin{tabular}{|c|c|c|}
\hline Country & $\begin{array}{l}\text { Average nutrient } \\
\text { level } \mu \mathrm{g} / \text { day }\end{array}$ & $\begin{array}{l}\text { Individual nutrient } \\
\text { level } \mu \mathrm{g} / \text { day }\end{array}$ \\
\hline Australia and New Zealand ${ }^{a}$ & 520 & 600 \\
\hline $\begin{array}{l}\text { Austria, Germany and } \\
\text { Switzerland }\end{array}$ & & 600 \\
\hline Denmark, Ireland and Sweden ${ }^{a}$ & & 500 \\
\hline European Community & & 400 \\
\hline Mexico & & 750 (safe level) \\
\hline Poland ${ }^{a}$ & 520 & 600 \\
\hline The Netherlands ${ }^{a}$ & & 600 (safe level) \\
\hline$U S A^{a}$ & 520 & 600 \\
\hline$U K^{a}$ & & 300 \\
\hline $\mathrm{FAO} / \mathrm{WHO} / \mathrm{UNU}$ & 520 & 600 (safe level) \\
\hline
\end{tabular}

${ }^{\mathrm{a}} 400 \mu \mathrm{g}$ folic acid supplementation given in addition to folic acid requirements

Phe-free L-amino acid supplements may be poor, resulting in sub-optimal intake. Giving an additional $400 \mu \mathrm{g} /$ day of folic acid in the early weeks of pregnancy should apply in PKU as in healthy women. Vitamin B12 (including functional marker plasma homocysteine and/or methylmalonic acid) should be monitored to ensure that high intake of folic acid does not mask vitamin B12 deficiency [383].

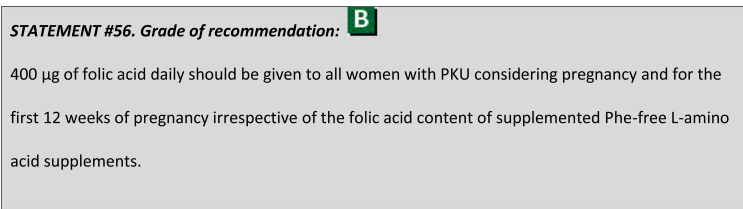

EPA and DHA Sub-optimal concentrations of EPA and DHA have been reported in maternal PKU [384] and traditional dietary treatment is low in $\alpha$-linolenic acid, arachidonic acid and without sources of EPA and DHA [268]. DHA (n-3) and arachidonic acid (n-6) are essential for foetal growth [385]. In healthy women, an increased supply of $n-3$ LC-PUFA during pregnancy reduces the risk of preterm birth before 34 weeks of gestation. Pregnant women should achieve an additional supply $\geq 200 \mathrm{mg}$ DHA/day, over and above the intake recommended for adult general health usually achieving a total intake $\geq 300 \mathrm{mg}$ DHA/day [386], and this should be given to all women considering pregnancy and during pregnancy in PKU. Many (but not all) Phe-free L-amino acid supplements contain supplemented DHA, and usually would supply $120-150 \mathrm{mg}$ of DHA for each $20 \mathrm{~g}$ protein equivalent. Assessment of fatty acid status could be considered pre-conceptionally or early in pregnancy and supplementation can be given if biochemical deficiency is demonstrated.

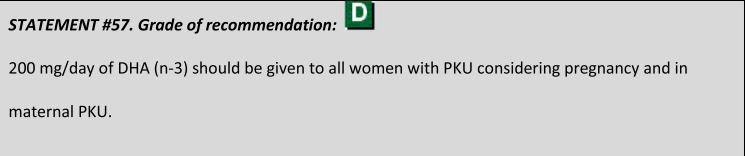

Nutrient monitoring It is essential that key nutrients are monitored pre-conception and at the start of pregnancy, with further monitoring recommended only if there are concerns about dietary adherence or biochemical/clinical deficiency has been noted earlier in the pregnancy. On a non-supplemented low Phe diet, intake of vitamin B12 and a decreased intake of vitamin B12 may contribute to an increased risk of congenital heart disease [359]. If vitamin B12 status is low at the start of dietary treatment it should be corrected with oral or intramuscular vitamin B12. Low selenium concentrations throughout pregnancy have also been noted in women with maternal PKU [186], without selenium supplements added to their Phe-free L-amino acid supplements. The MPKUCS revisited 28 pregnancies born with CHD. They had significantly higher blood Phe, lower proline, valine, methionine, isoleucine, leucine, lysine, arginine and lower red blood cell folate [360].

It is important to note that interpreting micronutrient blood markers is challenging during pregnancy due to the maternal, placental and fetal adaptations, which vary between individuals and are dependent on gestational age. These issues lead to reduced sensitivity and specificity of biomarkers particularly during late pregnancy and target blood ranges used for non-pregnancy may be inappropriate during pregnancy [387].

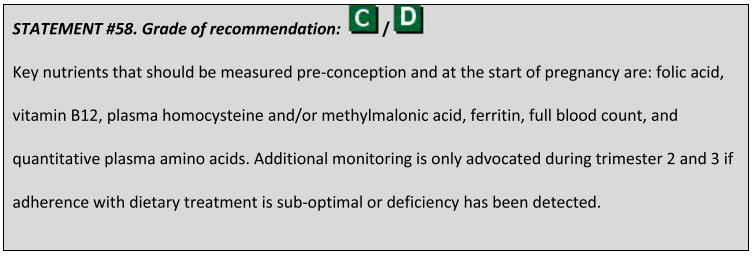

Breast-feeding and lactation Unaffected infants of maternal PKU women are able to metabolize the Phe contained in their mothers breast milk without difficulty $[102,388]$ and women with PKU should be encouraged to breast-feed their infants. The only contraindication is if the mother is treated with BH4 [389] because the product characteristics state that it is unknown if the drug or its metabolites are excreted in human breast milk. However, we encourage breastfeeding and do not consider there are contraindications for breastfeeding in (maternal) PKU, even with BH4. 
There is some suggestion that the Phe content of breast milk is higher than milk from healthy mothers. The Phe content of maternal breast milk is highest immediately post birth (up to $238 \mathrm{mg} / 100 \mathrm{ml}$ ) but decreases to 90 to $130 \mathrm{mg} / 100 \mathrm{ml}$ [390]. Bradburn et al. reported the Phe content of breast milk was $86 \mathrm{mg} / 100 \mathrm{ml}$ at day 6 postpartum and $74 \mathrm{mg} / 100 \mathrm{ml}$ at day 13 post-partum [388].

There is no published data about breast-feeding infants with PKU if mothers also have PKU. However, practical experience would suggest that breast-feeding is possible providing the same management principles as for all infants with PKU (i.e. a Phe-free formula is given pre-breast feeds) [391] are adopted.

Lactation is an exceptionally demanding nutritional state for the mother. Factors relating to sub-optimal maternal nutrition status during lactation include maternal age, quality of dietary treatment, lifestyle factors, and spacing of consecutive births [392]. Energy requirements of milk production are high with energy requirements considered to increase by $505 \mathrm{kcal} /$ day to $675 \mathrm{kcal} /$ day in the first 6 months of breast-feeding [393]. It is assumed that part of extra energy requirement will be met by fat stores that are laid down during pregnancy. An additional $15 \mathrm{~g} /$ day (approximate amount) protein to pre- pregnancy requirements should be provided [394].

There are no reports detailing Phe intake during lactation, probably because many women discontinue strict diet after pregnancy [395]. Blood Phe concentrations are likely to rise significantly associated with post-partum catabolism unless dietary energy intake and a low Phe intake is maintained. Phe requirements are likely to remain similar to pre-pregnancy requirements. It is important women are encouraged to return to a healthy weight post pregnancy. All women should receive regular nutritional support post pregnancy, and women who have discontinued dietary treatment may be particularly vulnerable to the effects of poor food choices.

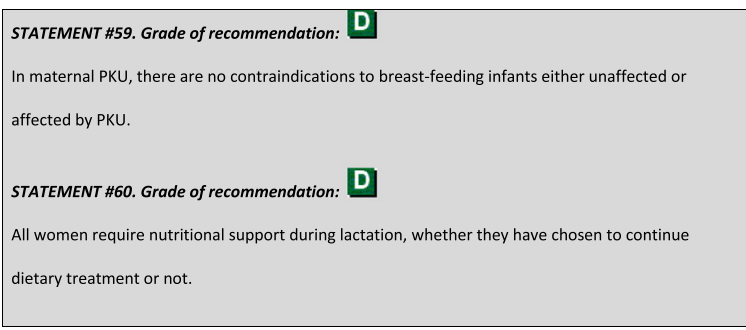

Dietary education programmes for maternal PKU The low Phe diet is challenging for women with PKU during pregnancy and women need much support and education about maternal PKU treatment. Many women have followed a normal diet for years and may never have managed their own dietary treatment. In PKU, poor
EF, e.g. sub-optimal planning and organizational skills, poor attention [70], and short-term memory [93], may affect the ability to self-manage a low Phe diet because of the day to day organization, technical skills and planning required [396]. Obtaining Phe-free L-amino acid supplements and low protein foods may be difficult. Poor dietary adherence was associated with the following maternal factors: younger women (25 and under), those with less formal education, (high school or less), and women using social assistance [397].

Women with a lower IQ require intensive practical help with dietary application. A 'Resource Mother' or diet support worker, providing practical assistance to women with PKU, has been proven to be very helpful by providing social support, enhancing positive attitudes toward the treatment and ensuring that necessary resources were in place [335]. This care is provided in patients own home. In a USA study, the Maternal PKU Resource Mothers Program matched mothers of children with PKU (Resource Mother) to women with PKU who were planning a pregnancy or who were already pregnant, with the aim of providing social support, enhancing positive attitudes toward the treatment and ensuring that necessary resources were in place. Women who received the services of a 'Resource Mother' attained metabolic control on average, 2 weeks sooner than women who did not participate [335]. Waisbren et al. [398] also found that strong social practical and emotional support from family and medical providers greatly increased the chance that a woman would start treatment before pregnancy.

Alternatively, some patients are admitted to the hospital for a 3-5 day intensive education. As well as receiving dietary and cooking advice, they are taught how to do their own blood Phe measurements [333].

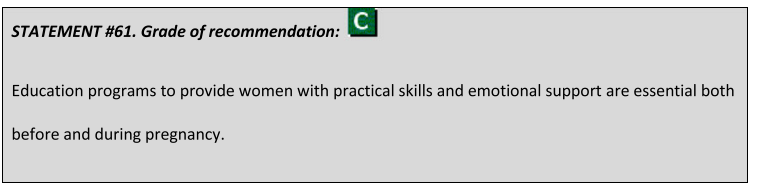

\section{Late diagnosed and untreated PKU}

Several definitions are used to describe untreated, late diagnosed or late treated children and adults with PKU. In this section the terms late diagnosed and untreated PKU are used. Late diagnosed refers to children diagnosed between the ages of 3 months to 7 years ( $\geq 3$ months - $<7$ years). Untreated PKU refers to patients untreated by 7 years of age and over. It is acknowledged that these definitions are arbitrary but improvement in IQ is rarely observed if treatment is started after the age of 7 years $[399,400]$.

There are many late diagnosed and untreated patients with PKU due to a lack of NBS, NBS failures and/or 
immigration of patients from countries without NBS or treatment [74, 401-404]. Some cases may not be diagnosed until adulthood, presenting with neurological complications (without severe neurocognitive impairment) possibly related to PKU $[405,406]$.

Untreated patients with severe intellectual disability and challenging behavioural problems have high support needs [407], and some may live in social welfare homes [408, 409]. An increase in life expectancy suggests the importance of their identification and the provision of long-term care planning [410]. Intervention with a low Phe diet may be beneficial [407]. Their overall rehabilitation program should not be different from individuals with other causes of intellectual disability.

\section{Late diagnosed PKU}

Late diagnosed PKU patients may significantly benefit from the introduction of a low Phe diet, which may improve intellectual performance [74, 411, 412]. Outcome is mainly influenced by age and developmental quotient/IQ at the start of treatment [399, 400]. Reversibility of IQ loss may occur especially in the first 4-6 years of life [74, 399, 400], although this has also been reported in an 8 year old child [413].

\section{Untreated PKU}

Untreated PKU patients even with severe intellectual disabilities may benefit from the introduction of a low Phe diet. Several case reports and cohorts describe changes in symptoms in untreated adults after commencement of dietary treatment [71, 399, 401, 413-426], although benefit is not seen in all patients [71, 414, 415, 418, 422, 424, 426, 427]. Mainly improvement of motor function (tremors/spasticity) and behaviour (less restless and irritable, more alert/responsive and less aggressive with decreased numbers and severity of self-injury behaviours) are described (Table 16). Giffin et al. [428] reported improvement in visual attention span in 2 out of 3 patients. Schuett et al. [429] recounted positive and negative results after diet commencement in 42 mildly and severely intellectually impaired patients concerning outcomes such as mood, hyperactivity, body weight and nausea/vomiting.

Brown et al. [430] determined that a low Phe diet in adults with previously untreated PKU resulted in economic benefit to the health service and society in general. Reduction in nursing time, hospitalizations, outpatient clinic visits and medications reduced mean annual costs [430].

\section{Dietary treatment and monitoring}

Before diet initiation, it is essential to consider the individual patient and their quality of life especially if they have severe intellectual disability and/or behavioural problems [74]. A low-Phe diet for previously untreated adults should be supervised by a team experienced in
Table 16 Expected positive outcome changes with Phe-restricted dietary treatment in untreated PKU patients

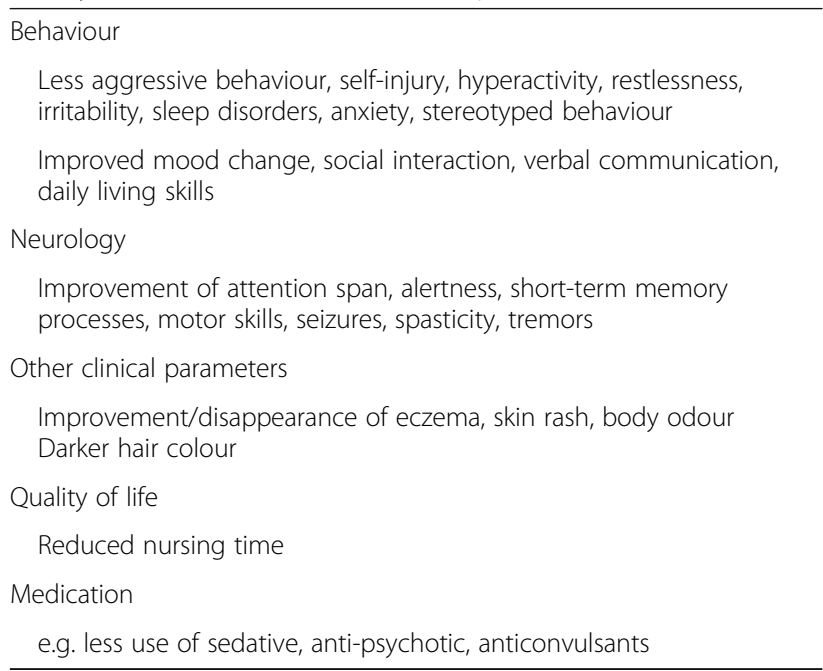

Reported in the literature of chapter 9.2 untreated PKU

the treatment of PKU. Care providers, family members and/or residential house staff members require instruction about the practicalities of dietary treatment. Potential management barriers should be identified. Care providers and families may consider that the diet is too restricted and so inappropriate [417]. Clear information should be given about the potential benefits of dietary treatment. A practical plan for stepwise introduction of a low Phe diet is published by Dolan et al. [417], and Hoskin [431]. Dietary management needs careful nutritional monitoring to optimize Phe, total protein energy and micronutrient intakes. Adjustment of Phe-free Lamino acid supplements may be necessary [127]. When treatment does improve patient behaviour and social interaction, adjustment of social and therapeutic programs is essential. Potential expected outcome changes are identified in Table 16.

Although not fully understood, in some patients post diet initiation, there may be deterioration of symptoms with increased frequency of aggressive behaviour and recurrence of seizures [426]. It may be necessary to consider discontinuation of dietary management. It is not possible to predict which patients will respond to a low Phe diet and the first clinical or behaviour changes may not occur for weeks or even months. However, diet discontinuation should be considered only if there is no clinical or behavioural improvement after 6 months, providing blood Phe levels have consistently been maintained within target range. Videotaping can help to record changes in behaviour.

Phe monitoring is recommended weekly at treatment commencement but should follow standard recommendations for monitoring once blood Phe levels have stabilised within target range. Target Phe levels and nutritional 
follow-up recommendations are discussed in the chapter treatment goals and follow up. Although it is not clear what the optimal target blood level should be in untreated adult patients, we recommend $<600 \mu \mathrm{mol} / \mathrm{l}$. Dolan et al. [417] and Koch et al. [399] reported upper target Phe levels of $600 \mu \mathrm{mol} / \mathrm{l}$ [417] and $720 \mu \mathrm{mol} / \mathrm{l}$ [399] respectively.

\section{Other therapies}

There are limited reports on the use of additional therapies in later or undiagnosed PKU. Kalkanoglu et al. [432] demonstrated in a double-blind cross-over study improved concentration, awareness and less selfinjurious behaviour in 14 of 19 untreated adults by LNAA supplementation. Vernon et al. [433] reported one BH4-responsive untreated adult with PKU with a baseline plasma Phe of $1255 \mu \mathrm{mol} / \mathrm{l}$. On BH4 treatment he showed significant behavioural improvements, resulting in fewer behavioural problems and increased social interactions. Treatment with $\mathrm{BH} 4$ should be considered for any responsive patient independent of their mental capacity. BH4-treatment is discussed in the chapter pharmacological treatment and emerging therapies.
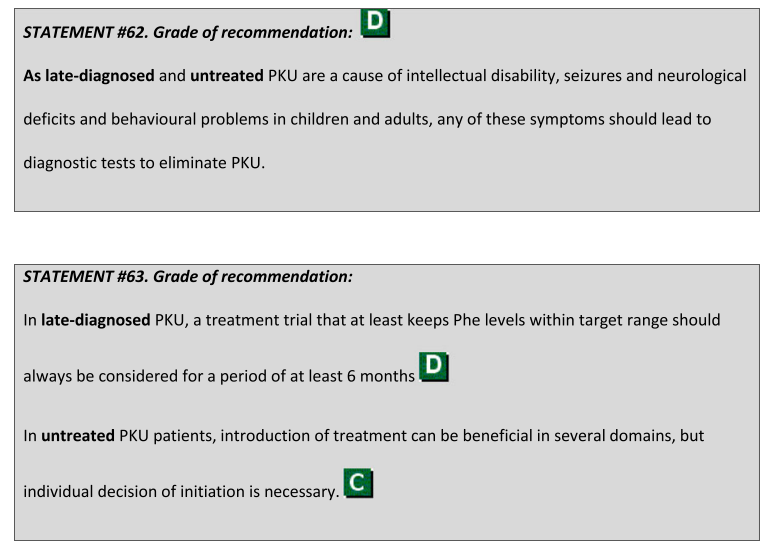

\section{Adherence}

Poor treatment adherence is common in any chronic disease. Counselling and education is often recommended to improve adherence. Research indicates that whilst knowledge is necessary for adherence, it is not a strong predictor of adherence [198, 434]. Short-term gain in knowledge can be achieved by intervention programs such as summer camp, but adherence and metabolic control do not improve after interventions [435-437]. Changes in attitude and motivation may be more effective [438].

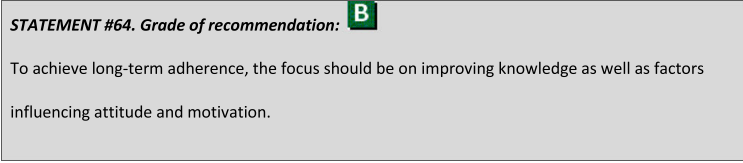

Whereas traditionally the metabolic team prescribed and monitored treatment in PKU, this is now commonly done in partnership with the caregivers and patients. It is recognised that it is valuable to provide personcentred care, providing advice and support that is focussed on the individual rather than being judgmental about poor adherence [10]. This is particularly important with adolescents and adult patients. Adult patients often prefer management that interferes with normal life as little as possible. They are expected to take responsibility for their own health with the metabolic team providing the right information, tools and motivational encouragement [10]. Unfortunately, some adult patients no longer attend hospital appointments for follow-up care, and one of the most difficult challenges is re-engagement of this patient group. They may have poor understanding of their condition, its potential consequences and retain unpleasant memories of dietary treatment. Reaching out to this cohort necessitates collaborative efforts of PKU clinics and national patient advocacy organizations, possibly using social media, to help nurture them to return to clinical follow-up [439].

In children under 12 years of age, although uncommon, there are cases in which parents or caregivers refuse to engage with healthcare professionals. Common signs of poor adherence in early childhood include: persistently poor Phe control, failure to engage with health professionals (e.g. non-attendance at clinic appointments, non-response to telephone calls), and sporadic return of blood Phe spots [440]. Known factors associated with chronic poor adherence that affects parenting capacity includes lack of intellectual abilities of parents, alcoholism, drug abuse and mental health issues [441] and other social issues including financial issues and chronic illness of parents. Health care providers have a legal obligation to protect and care for each child in their clinic and recommendations for action when a child has chronic poor metabolic control are summarised in statement \#65:

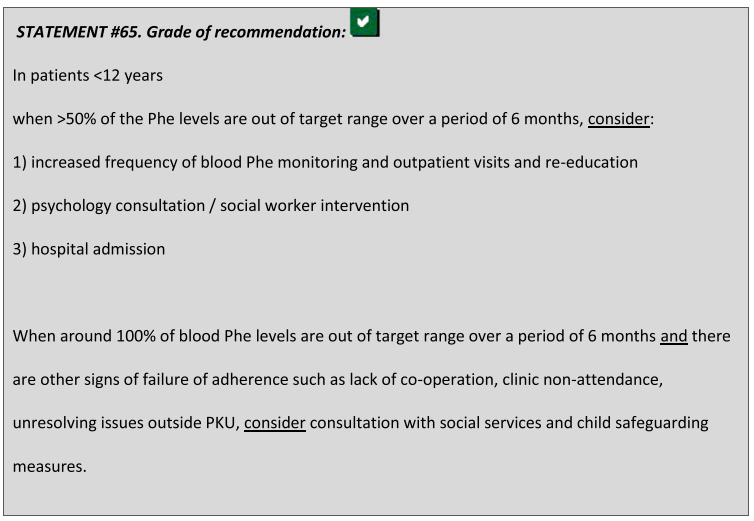




\section{Pharmacological treatment and emerging therapies BH4 treatment}

$\mathrm{BH} 4$, also known as sapropterin dihydrochloride (the active compound in the commercial drug) is used to treat a subset of PKU patients with PKU [193, 311, 433, 442453]. Patients with high residual activity of the PAH enzyme have a greater probability of $\mathrm{BH} 4$ response, but a minority of patients with classical PKU also may benefit from BH4 treatment [193, 194, 442, 443, 445, 449, 453]. Recently, efficacy and safety of BH4 has been demonstrated in children $<4$ years of age which has led to European approval for BH4 in this age category [454-457]. $\mathrm{BH} 4$ is still unavailable in some European countries.

Two systematic reviews have summarised the efficacy and safety of BH4. Somaraju et al. [458] reviewed 2 randomised controlled trials that were led respectively by Levy et al. [447] and Trefz et al. ([459], whereas Lindegren et al. [448] included the following additional studies/reports: one uncontrolled open-label study, one prospective cohort study, and several case series. Both systematic reviews concluded there is short-term evidence to demonstrate that $\mathrm{BH} 4$ is effective in reducing blood Phe concentrations and increasing Phe tolerance in BH4-responsive PKU patients. Also no serious adverse events were reported. Several uncontrolled open-label studies and case series support a significant reduction of blood Phe levels [193, $194,311,433,442,444-451,453-455,458-464]$ and increased Phe tolerance [193, 194, 311, 433, 443-446, 448452, 454, 455, 458, 459, 463-465]. These benefits have also been replicated in longer-term (investigated up to 5 years) studies [193, 194, 443, 445, 446, 451, 454, 455, $463,465]$. Furthermore, less variability in blood Phe control has been described in 3 descriptive papers [311, 454, 460]. Current data suggests that with BH4 treatment cognition and behaviour issues may improve but, possibly, more importantly they do not deteriorate [155, 183, 466]. The same applies for quality of life $[58,165]$. At present, studies have not reported long-term neurocognitive outcome, behaviour and quality of life with $\mathrm{BH} 4$ treatment.

The cost-effectiveness of BH4 is not established, especially when dietary treatment and Phe-free L-amino acid supplements are still required.

BH4-responsiveness should be determined on an individual case basis. The degree of responsiveness will be characterised by the extent of improvement in biochemical control and increase in natural protein intake. We define BH4-responsiveness as 'establishing an increase in natural protein tolerance of $\geq 100 \%$ with blood Phe concentrations remaining consistently within the target range'. BH4-responsiveness can also be defined by improved metabolic control ' $>75 \%$ of blood Phe levels remaining within target range without any decrease in natural protein intake associated with BH4 treatment'. BH4 should only be prescribed in cases of proven $\mathrm{BH} 4$-responsiveness which is established by a treatment trial (chapter 11.2). BH4 should be withdrawn if blood Phe levels consistently exceed the upper target range and there is no improvement associated with any increase in $\mathrm{BH} 4$ dosage. If nutritional status deteriorates e.g. obesity or development of nutritional deficiencies, discontinuation of $\mathrm{BH} 4$ treatment should be considered.

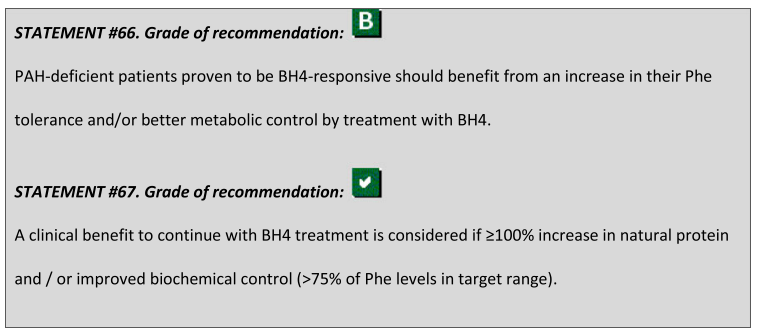

BH4 and pregnancy Since drug studies in pregnancy are not feasible, experience is based on a small number of case reports and 2 small cohort studies. Therefore, no prospective data is available regarding the indication, dose and management of BH4 during pregnancy. In a European cohort study, 3 patients received BH4 preconception, and 5 patients commenced treatment during pregnancy [467]. In a USA cohort study, 15 patients were administered $\mathrm{BH} 4$ prior to pregnancy, and only 1 patient received $\mathrm{BH} 4$ post-conception $[468,469]$. Overall, the dosage varied between 4 and $20 \mathrm{mg}$ per $\mathrm{kg}$ of body weight. The Kuvan ${ }^{\circ}$ Adult Maternal Paediatric European Registry reported 4 pregnancies, with varying BH4 doses between 3 and $17 \mathrm{mg}$ per $\mathrm{kg}$ of body weight. No foetal development problems or adverse events related to the pregnancies were observed [465].

Case descriptions show that $\mathrm{BH} 4$ assists in lowering blood Phe levels to within target range. All the infants exposed to BH4 during pregnancy had favourable outcomes, except in 1 case when the mother had very high Phe levels in early pregnancy and was given $\mathrm{BH} 4$ as a rescue treatment. $\mathrm{BH} 4$ treatment can be given during pregnancy, but only if women are known to be BH4responders and dietary treatment alone is unsuccessful in achieving target blood Phe control. Potential responsiveness can be assessed by genotyping and/or a $\mathrm{BH} 4$ loading test [35].

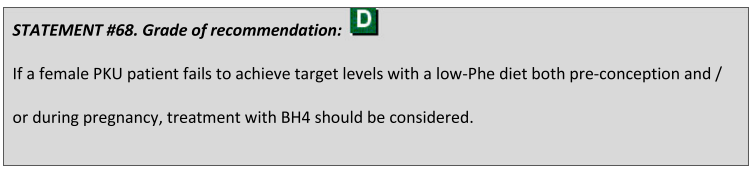

BH4 and untreated adults This is discussed in the chapter on untreated adults. 


\section{$\mathrm{BH} 4$ loading test and treatment trial}

Before treating patients with $\mathrm{BH} 4$, assessment of $\mathrm{BH} 4-$ responsiveness is essential. Determination of BH4responsiveness can be done by genotype and/or $\mathrm{BH} 4$ loading tests. As already reported in chapter 4.3, the genotype may predict or exclude to some degree BH4responsiveness [32-34]. Every patient except if there are 2 null-variants justifies a $\mathrm{BH} 4$ loading test. If 2 BH4responsive variants are identified, a treatment trial without a BH4 loading test can be commenced.

The short-term loading test take up to $48 \mathrm{~h}$ in Europe and 28 days in USA, whereas the treatment trial in potential BH4-responders (after a positive short-term test or genotype in line with known BH4-responsiveness) may occur over a few weeks or even months [470]. Bernegger et al. [471] described a $24 \mathrm{~h} \mathrm{BH} 4$ loading testing using $20 \mathrm{mg} / \mathrm{kg}$ to differentiate between BH4responders and non-responders. Extension to $48 \mathrm{~h}$ and repeated $\mathrm{BH} 4$ administration seems to be useful to detect slow responders and responsiveness in more severe phenotypes [472]. The utility of a $48 \mathrm{~h}$ test has been confirmed in a study of 177 patients treated with $20 \mathrm{mg}$ BH4/ $\mathrm{kg} /$ day [35].

At present, the exact procedure of the $\mathrm{BH} 4$ loading test differs among countries, also depending on laboratory availability. Blau et al. [470] and Singh et al. [450] published protocols for $\mathrm{BH} 4$ loading tests. The $24 \mathrm{~h}$ test in newborns can detect BH4-deficiencies in addition to BH4-responsiveness in PAH patients [473]. The arbitrary responsiveness definition of a $>30 \%$ reduction in blood Phe appears to be a good compromise between sensitivity and specificity for the initial screening test. Individual patient characteristics should be considered when interpreting results, especially in patients with low baseline Phe levels [474].

Every positive $\mathrm{BH} 4$ loading test and gene variants analysis refers to a potential BH4-responsiveness. Longterm response should be proven in a treatment trial adjusting the $\mathrm{BH} 4$ dosing, natural protein intake and Phe-free L-amino acid supplements. The starting dose in a treatment trial is $10-20 \mathrm{mg}$ per $\mathrm{kg}$ of body weight and can be adapted during the trial. This process may require several weeks to months. There is a lack of studies that have addressed the long-term treatment $\mathrm{BH} 4$ dose, the natural protein tolerance and supplementation with Phe-free L-amino acids. When adapting diet with $\mathrm{BH} 4$, Singh et al. [450] advised to increase the natural protein first, followed by reduction in Phe-free L-amino acid supplementation. An additional step should include a reduction in the $\mathrm{BH} 4$ dose. It is important to maintain blood Phe concentrations in the target range with $\mathrm{BH} 4$ treatment and diet relaxation. It is also important to anticipate that patients may not always make the healthiest food choices when given some dietary freedom. Increasing the natural protein (from non-animal food sources) and decreasing the Phe-free L-amino acid supplementation may even result in nutritional deficiencies $[75,452]$.

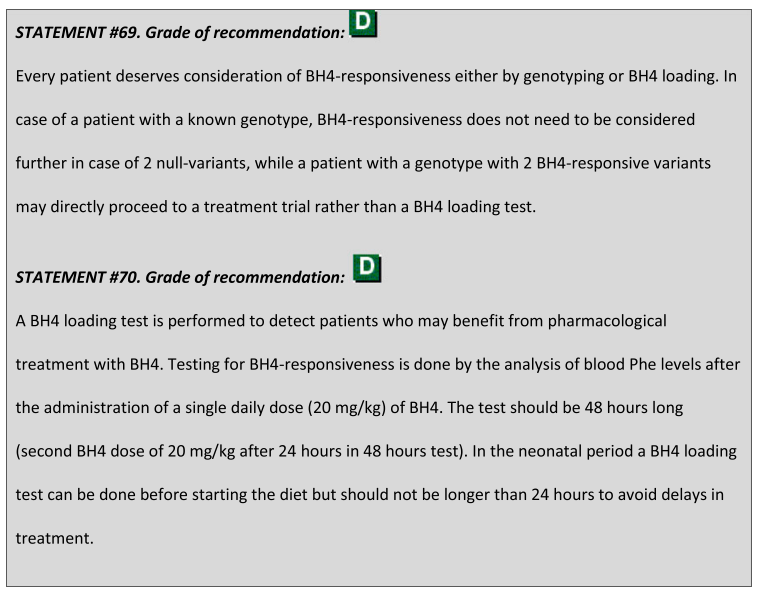

\section{Emerging therapies}

Effectiveness of PKU treatment is demonstrated by any of the following objective measures: reduction in Phe blood concentrations, increase in natural protein tolerance, improvement in neuropsychological testing, improved nutritional staus and better quality of life. Special considerations should be made for patients at different ages and special situations such as pregnancy or breast-feeding.

A possible enzyme replacement therapy using PEGPhenylalanine-Ammonia Lyase (PAL) or Pegvaliase is under investigation. PEG-PAL clinical phase II trials have proven short-term reduction in the Phe blood concentrations in adult PKU patients, but further studies are required to observe long-term effectiveness and safety [475]. Results of a phase III extension study (NCT01819727) are awaited. Gene therapy and therapeutic liver repopulation are being investigated in murine models only [476, 477], and larger animal PKU models and human studies are being developed.

\section{Patients' view}

There are several papers discussing patients' and parents' coping strategies and barriers to effective management. Awizus et al. [478] interviewed 11 parents of children aged 8 years with PKU. Parents were shocked by the diagnosis, had emotional and adjustment problems and were commonly in conflict between the task of attaining acceptable blood Phe levels and the guilt they experienced when they deviated from the dietary rules to meet their children's demands. In another study parents described their children with PKU as feeling different from peers [479] whereas patients described themselves as 'healthy' or 'normal' [480, 481]. Di Commo et al. [480] concluded from 20 patients 
with PKU, that they consider PKU more as a potential risk rather than an actual disease and that adherence seems connected with highly internalized behaviour rather than with perception of adverse effects. Some PKU patients avoid social occasions where food is shared because of fear of stigmatization. Patients $(n=47)$ experience a paradox, either they feel normal but isolated from the social context, or are different while participating in the convivial aspects of the social settings [481].

Primary obstacles to successful management of PKU identified by parents of 32 PKU children were time constraints, stress associated with food preparation, record keeping, and social life restrictions imposed by PKU. About two-thirds agreed that a home-monitoring blood device was desirable to ease the burden of management [482].

Bernstein et al. [483] studied the effectiveness of educational tools perceived by clinicians, parents and patients. They found a discrepancy between patient and clinician views regarding the effectiveness of nutrition education. Patients concluded that their families were the most effective educators whilst parents responded they felt one-on-one counselling was the most effective educational tool [483].

Hagedorn et al. [11] reported the minimum standard of care requested by patients/caregivers and the chief requirements were: uniform treatment/management goals; care by a multidisciplinary team (physician, dietician/ nutritionist and psychologist); and access to care, $\mathrm{BH} 4$, and special dietary products [11].

In order to represent the interest of patients, national patient organizations are recommended.

\section{Implementation/impact of guidelines}

Distributing the European PKU guidelines may increase awareness of their presence, but in itself will generally not lead to behaviour change [484]. Interactive educational interventions and reminders (when used sparingly) to health care providers are considered to be effective [484]. Potential barriers to behaviour change are lack of motivation, inadequate facilities and resources. The impact of these guidelines on daily care will differ among countries. Change of target blood Phe levels and the recommendation to follow up and treat patients for life may have impact on the intensity of care. The same applies to other recommendations such as the follow up of bone mineral density, nutritional status, neurocognition and frequency of Phe measurement and outpatient clinic visits.

Some recommendations can have impact on the health care budget. For example, in some centres there may be a need for additional staff in their paediatric healthcare team and/or for transition to adulthood. Reimbursement for $\mathrm{BH} 4$ treatment is not established in all countries.

The aim is to have the entire guideline updated in 5 years. The actual impact of the guidelines on change in healthcare will be evaluated by questionnaires.

\section{Future requirements and research}

It is evident that many of these guideline statements have not yet been introduced into clinical practice by several European centres and it is also clear that various barriers, including financial hurdles, may impede the speed of change. Unmet needs include identification and training of sufficient numbers of physicians with a broad interest in co-ordinating care for adult patients with IMD including PKU. We need to optimize PKU treatment both in adult and elderly care. The development of a device able to accurately measure and generate immediate blood Phe results for home monitoring (instead of home sampling) is likely to change management practices. It will decrease metabolic laboratory time, and will dramatically reduce the time between blood sampling and obtaining a blood Phe result, and so assist and motivate patients to achieve target blood Phe target ranges more easily. The establishment of the expert reference network (ERN) may lead to patient treatment being directed by European designated expert centres with 'local' care provided by experienced treatment teams and may facilitate the use of international databases by which studies on larger populations can be performed. There is an ongoing need for metaanalysis relating outcome to metabolic control during childhood, adolescence, and adulthood, while the need for more sophisticated statistics in such studies is underestimated.

Future research in PKU may target the pathophysiology of brain dysfunction aiming to improve treatment strategies. These strategies may not only target the blood Phe concentrations, but also directly alter cerebral metabolism. They will aim to improve neuropsychological outcome and functioning as well as provide a better quality of life by decreasing the need for arduous dietary Phe restriction. Such treatments may include new drugs such as enzyme replacement therapy using PEG-PhenylalanineAmmonia Lyase (PAL) but other non-nutritional treatment options including gene therapy and therapeutic liver repopulation have not progressed beyond animal models.

Future research is necessary to identify the number of adults who experience clinical symptoms together with better characterisation and impact of sign and symptoms. More data is needed about the influence of metabolic control during adolescence and adulthood, particularly when childhood metabolic control is optimal. In addition, new strategies should be actively sought to re-engage adult patients who are no longer in active hospital follow up but who are at risk of mental health and executive function deficits.

Other future research topics include strategies to improve adherence particularly in adolescents and adulthood, efficacy of enzyme replacement therapy, effectiveness of GMP and LNAA, usefulness of biochemical markers such as Phe variability, the Phe: Tyr ratio as well as the ratio of Phe to other LNAA, new 
(long-term) biomarkers, defining the optimal lower target Phe levels, bioavailability of micronutrients in Phe-free L-amino acid supplements and the functionality and long-term side effects of Phe-free L-amino acid supplements in PKU.

\section{Conclusion}

These first European guidelines are the result of a 3 year process based on the AGREE and SIGN methodology as PKU management differs accros Europe. The level of evidence of most recommendations is $\mathrm{C}$ or $\mathrm{D}$. Although study designs and patient numbers are sub-optimal, many statements are convincing, important and relevant, and may set the benchmark for improving outcome in PKU patients. Key recommendations which should be prioritised for implementations mainly relate to treatment initiation, target Phe levels for treatment, and follow-up. Minimum requirements regarding management and follow-up of PKU patients are formulated. Knowledge gaps are identified that require further research in order to direct better future care. Future research should focus on the pathophysiology of brain dysfunction aiming to improve treatment strategies and the impact of metabolic control during adolescence and adulthood. These guidelines are aimed to standardize care and do determine a course of action, but are not mandatory. The authors of these guidelines are willing to update these guidelines based on the highest quality evidence available.

\section{Definitions}

\begin{tabular}{ll}
\hline Concurrent Phe level & The Phe level measured at or close \\
& to the day of outcome assessment. \\
& The European Society for \\
ESPKU & Phenylketonuria and Allied \\
& Disorders is the umbrella \\
& organization of national and \\
regional associations from about 30 \\
countries established by parents.
\end{tabular}

Executive functioning

High risk pregnancy

Late treated and/or untreated adults

Lifetime Phe levels
(Continued)

\begin{tabular}{ll}
\hline & $\begin{array}{l}\text { (semi)annual mean Phe levels. Also } \\
\text { referred to as historical Phe levels. } \\
\text { Maternal PKU syndrome }\end{array}$ \\
& $\begin{array}{l}\text { The teratogenic effects of elevated } \\
\text { maternal phenylalanine levels } \\
\text { during pregnancy to the foetus. }\end{array}$ \\
Thenylalanine tolerance & The amount of phenylalanine (mg/ \\
& plasma phenylalanine \\
& concentrations within the target \\
& range. \\
& This may also be described as \\
& natural protein tolerance expressed \\
& as g/day taking a phenylanine \\
content in natural protein as $50 \mathrm{mg}$ \\
phenylalanine/g natural protein.
\end{tabular}

Protein requirements

The lowest level of dietary protein intake that will balance the losses of nitrogen from the body, and thus maintain the body protein mass in persons at energy balance with modest levels of physical activity.

Protein substitutes (phenylalaninefree L-amino acid supplements and low phenylalanine glycomacropeptide protein)

Protein replacement/substitutes are essential to prevent protein deficiency and optimize metabolic control. Protein substitutes are mainly sourced from phenylalaninefree $L$-amino acids supplements and less commonly from low phenylalanine glycomacropeptide.

Potential tetrahydrobiopterin $(\mathrm{BH} 4)$ responsiveness

$>30 \%$ reduction in blood phenylalanine in a $\mathrm{BH} 4$ loading test or $2 \mathrm{BH} 4$ responsive variants. Longterm $\mathrm{BH} 4$-responsiveness should be proven in a treatment trial adjusting the $\mathrm{BH} 4$ dosing, natural protein intake and phenylalanine-free $\mathrm{L}$ amino acid supplement.

Tetrahydrobiopterin (BH4)

Cofactor of the phenylalanine hydroxylase. $\mathrm{BH} 4$ also acts as a chaperone molecule in phenylalanine hydroxylase-deficient patients harbouring specific gene variants. $\mathrm{BH} 4$ is also a cofactor of tyrosine and tryptophan hydroxylases and plays an important role in the conversion of $\mathrm{L}$-arginine to $\mathrm{ni}$ tric oxide (NO) by nitric oxide synthases (NOS).

Tetrahydrobiopterin $(\mathrm{BH} 4)$ deficiencies

Defects in $\mathrm{BH} 4$ metabolism (either synthesis or regeneration) result in a deficiency of $\mathrm{BH} 4$. These include 3 known defects for synthesis and 2 for regeneration.

Tetrahydrobiopterin (BH4) responsiveness

In this report it is defined as an increase of $\geq 100 \%$ in natural protein and/or improved biochemical control ( $>75 \%$ of phenylalanine levels in target range) on a dose of $\mathrm{BH} 4$ that ranges between 1 and 20 mg perkg of body weight (with a maximum dose of 1000 or $1400 \mathrm{mg} / \mathrm{day}$ in some cntries). 


\section{Abbreviations}

ADHD: Attention deficit hyperactivity disorder; AGREE: Appraisal of guidelines for research and evaluation; APL: Assigned blood Phe levels; BDI: Beck depression inventory; $\mathrm{BH}$ : Tetrahydrobiopterin; BMD: Bone mineral density; BMI: Body mass index; CBCL: Child behaviour checklist; CHD: Congenital heart disease; DACH-RDA: Regular daily allowance recommended for the German speaking countries Germany, Austria and Switzerland; DBS: Dried blood spots; DHA: Docosahexaenoic acid; DHPR: Dihydropteridine reductase; DXA: Dual-energy X-ray absorptiometry; EF: Executive function; EPA: Eicosapentaenoic acid; ERN: Expert reference network; ESPKU: European Society of Phenylketonuria and Allied Disorders; ETPKU: Early treated PKU; FAO: Food and Agriculture Organization of the United Nations; GMP: Glycomacropeptide; GTPCH: GTP cyclohydrolase I; HPA: Hyperphenylalaninemia; HRQoL: Health related quality of life; IMD: Inherited metabolic disorder; IQ: Intelligence quotient; ISCD: International Society for Clinical Densitometry; IUGR: Intrauterine growth retardation; LC-PUFA: Long chain polyunsaturated fatty acids; LNAA: Large neutral amino acids; MBD: Mineral bone disease; MHP: Mild hyperphenylalaninemia; MPKUCS: Maternal PKU collaborative study: MRI: Magnetic resonance imaging; NBS: Newborn screening; PAH: Phenylalanine hydroxylase; PAL: Phenylalanine ammonia lyase; PCD: Pterin-4a-carbinolamine dehydratase; PEG: Polyethylene glycol; Phe: Phenylalanine; PKU: Phenylketonuria; PTH: Parathyroid hormone; PTPS: 6-pyruvoyl-tetrahydropterin synthase; RDA: Recommended dietary allowances; RNI: Reference nutrient intakes; SIGN: Scottish intercollegiate guidelines network; SR: Sepiapterin reductase; Tyr: Tyrosine; UNU: United Nations University; USA: The United States of America; WHO: World Health Organization; WM: White matter; WMA: White matter alteration

\section{Acknowledgments}

We gratefully acknowledge the external consultants of these guidelines. We acknowledge obstetrician Dr. Jerzy Giżewski who actively contributed during 1 of the group sessions focusing on Maternal PKU. Finally we would like to thank the external reviewers S. Beblo, G. Berry, M. Bik-Multanowski, M. Cleary, T. Coşkun, H. Gökmen-Özel, J. Häberle, R. Lachmann, H. Levy, Y. Okano, I. Schwartz, J. Zeman and patient organization ESPKU. Development of these guidelines was financial supported by the European Society of Phenylketonuria and Allied Disorders (ESPKU). One group and 1 plenary meeting were supported by Excemed.

\section{Funding}

We received a grant from the ESPKU so that we were able to appoint a project assistant (AvW). The ESPKU had no opportunity to influence the writing process.

\section{Availability of data and materials}

Data sharing not applicable to this article as no datasets were generated or analysed during the current study.

\section{Authors' contributions}

KA Member of working group A; nutritional treatment and nutritional/ biochemical follow-up. Main topic: large neutral amino acids. ABQ Member of working group E; diagnosis of PKU and Drugs in PKU. Main topic: emerging therapies. NB Member of working group E; diagnosis of PKU and Drugs in PKU. Main topics: diagnosis, differential diagnosis, genotyping, $\mathrm{BH} 4$ loading test. AMB Member of working group C; psychosocial outcome and adherence. Main topics: psychosocial functioning (including quality of life), mental health. AB Member of working group B; Neurocognitive outcome including imaging. Main topic: magnetic resonance imaging. JC Member of working group $B_{i}$ Neurocognitive outcome including imaging. Main topic: target Phe levels, biochemical markers, neurocognitive functioning. FF Member of working group A; nutritional treatment and nutritional/biochemical follow-up. Main topics: bone mineral density, oxidative stress, biochemical follow-up. MG Member of working group D; Adult and Maternal PKU, late diagnosed and untreated PKU. Main topics: maternal PKU, late diagnosed and untreated PKU. SCJH Member of working group B; Neurocognitive outcome including imaging. Main topic: target Phe levels, biochemical markers, neurocognitive functioning. SK Member of working group C; psychosocial outcome and adherence. Main topics: frequency of visits/Phe level measurement. VL Member of working group B; Neurocognitive outcome including imaging. Main topic: magnetic resonance imaging. AM Member of working group A; nutritional treatment and nutritional/biochemical follow-up. Main topics: dietary treatment. Contributed in Group D on topics: dietary treatment in maternal PKU, untreated/late treated adults. Edited manuscript. FM Member of working group D; Adult and Maternal PKU, late diagnosed and untreated PKU. Main topics: Adult PKU. ACM Member of working group E; diagnosis of PKU and Drugs in PKU. Main topic: BH4 treatment. MR Member of working group C; psychosocial outcome and adherence. Main topics: adherence. Contributed in group D on topic untreated/late treated adults. FJS Lead of project. Second lead writer of manuscript. Contributed to group B \& E in topics, initiation of treatment, methods of measuring Phe. Chaired plenary discussions. FT Member of working group D; Adult and Maternal PKU, late diagnosed and untreated PKU. Leading topics: maternal PKU, late diagnosed and untreated PKU. JHW Member of working group A; nutritional treatment and nutritional/ biochemical follow-up. Leading topics: Parenteral nutrition. Edited manuscript. AMJVW Project assistant. Lead writer of manuscript. Assisted all working groups in literature search, selection and grading. Processed the data. Co-chaired plenary discussions. All authors contributed in plenary discussions, and co-wrote, read and approved the manuscript.

\section{Ethics approval and consent to participate}

Not applicable.

\section{Consent for publication}

Not applicable.

\section{Competing interests}

K. Ahring was/is a member of the European Nutrition Expert Panel (Merck Serono international, Biomarin).

A. Bélanger-Quintana has received honoraria as a speaker from Nutricia, Vitaflo International, Merck Serono and Recordati, and is a member of the European Nutrition Expert Panel (Merck Serono international, Biomarin), the Sapropterin Advisory Board (Merck Serono international, Biomarin), and KAMPER Advisory Board (Merck Serono international, Biomarin).

N. Blau was/is a member of Merck-Serono and Biomarin Scientific Advisory Board for PKU and has received grants and honorarium from Merck-Serono and BioMarin

A. Bosch was/is a member of Scientific Advisory Boards for Phenylketonuria that are supported by Merck-Serono SA, Biomarin and by Nutricia, and has received grants and honorarium from Merck-Serono and Nutricia.

A. Burlina was/is a member of Scientific Advisory Boards for Phenylketonuria that are supported by Merck-Serono SA, Biomarin and by Nutricia.

J.Campistol has received honoraria as a consultant and/or speaker from Merck-Serono SA and Nutricia/Danone.

M. Giżewska was/is a member of the Scientific Advisory Board for Phenylketonuria that was supported by Merck-Serono SA, and Biomarin and received honoraria as a consultant and/or speaker for Merck Serono SA and Nutricia.

F. Feillet was/is a member of Scientific Advisory Boards for Phenylketonuria (Merck-Serono SA, Biomarin) and has received honoraria from Merck-Serono, Nutricia/Danone and Excemed.

S.C. J. Huijbregts has participated in strategic advisory boards and received honoraria as a consultant and/or speaker for Merck Serono SA, Biomarin and Nutricia.

S. Kearney received honoraria from Merck Serono and Biomarin.

A. MacDonald has received research funding and honoraria from Nutricia, Vitaflo International, Merck Serono, chaired/chairs the European Nutrition Expert Panel (Merck Serono international and later Biomarin), was/is a member of the Sapropterin Advisory Board (Merck Serono international, Biomarin), and is a member of the Advisory Board Element (Danone-Nutricia). F. Maillot has received honoraria as a consultant and/or speaker from Merck Serono SA, Nutricia and Arla Foods.

A.C. Muntau was/is a member of Scientific Advisory Boards for Phenylketonuria (Merck Serono SA, Arla Foods, Biomarin); has received research funding from Nutricia, Vitaflo International, Merck Serono and has received honoraria from Merck Serono and Arla Foods.

M. van Rijn was a member of the European Nutritionist Expert Panel in PKU (until 2015), is a member of the ELEMENT (Leading Education in Metabolic Error Nutritional Therapy) Steering Committee for Nutricia International, and has received grants and fees for educational and research activities from Nutricia International and Orphan Europe.

F.J. van Spronsen was/is a member of Scientific Advisory Boards for Phenylketonuria (Merck-Serono SA, Arla Foods, Biomarin) and chairs the 
Scientific Advisory Board for metabolic diseases of Nutricia, has received grants from Nutricia and Merck-Serono SA), and honoraria from Merck-Serono SA, Vitaflo International, Nutricia/Danone, and Excemed.

F. Trefz received grants from Vitaflo and honoraria as a speaker from Merck-Serono SA

A. van Wegberg received a grant from the ESPKU.

The other authors declare no competing of interest.

\section{Publisher's Note}

Springer Nature remains neutral with regard to jurisdictional claims in published maps and institutional affiliations.

\section{Author details}

'Division of Metabolic Diseases, Beatrix Children's Hospital, University Medical Center Groningen, PO BOX 30.001, 9700 RB Groningen, The Netherlands. 2Dietetic Department, Birmingham Children's Hospital, Birmingham, UK. ${ }^{3}$ Department of PKU, Kennedy Centre, Glostrup, Denmark. ${ }^{4}$ Metabolic Diseases Unit, Department of Paediatrics, Hospital Ramon y Cajal Madrid, Madrid, Spain. ${ }^{5}$ University Children's Hospital, Dietmar-Hoppe Metabolic Centre, Heidelberg, Germany. ${ }^{6}$ University Children's Hospital Zürich, Zürich,

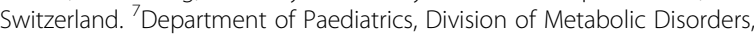
Academic Medical Centre, University Hospital of Amsterdam, Amsterdam, The Netherlands. ${ }^{8}$ Division of Inherited Metabolic Diseases, Department of Paediatrics, University Hospital of Padova, Padova, Italy. ${ }^{9}$ Neuropaediatrics Department, Hospital Sant Joan de Déu, Universitat de Barcelona, Barcelona Spain. ${ }^{10}$ Department of Paediatrics, Hôpital d'Enfants Brabois, CHU Nancy, Vandoeuvre les Nancy, France. ${ }^{11}$ Department of Paediatrics, Endocrinology, Diabetology, Metabolic Diseases and Cardiology of the Developmental Age Pomeranian Medical University, Szczecin, Poland. ${ }^{12}$ Department of Clinical Child and Adolescent Studies-Neurodevelopmental Disorders, Faculty of Social Sciences, Leiden University, Leiden, The Netherlands. ${ }^{13}$ Clinical Psychology Department, Birmingham Children's Hospital, Birmingham, UK. ${ }^{14}$ Department of Paediatrics, Child Neurology and Psychiatry, Sapienza University of Rome, Via dei Sabelli 108, 00185 Rome, Italy. ${ }^{15} \mathrm{CHRU}$ de Tours, Université François Rabelais, INSERM U1069, Tours, France. ${ }^{16}$ University Children's Hospital, University Medical Centre Hamburg-Eppendorf, 20246 Hamburg, Germany. ${ }^{17}$ Department of Paediatrics, University of Heidelberg, Heidelberg, Germany. ${ }^{18}$ Medicine, Manchester Academic Health Sciences Centre, Central Manchester University Hospitals NHS Foundation Trust, Manchester, UK.

Received: 3 March 2017 Accepted: 11 July 2017 Published online: 12 October 2017

\section{References}

1. Blau N, van Spronsen FJ, Levy HL. Phenylketonuria. Lancet. 2010;376(9750): 1417-27.

2. de Groot MJ, Hoeksma M, Blau N, Reijngoud DJ, van Spronsen FJ. Pathogenesis of cognitive dysfunction in phenylketonuria: review of hypotheses. Mol Genet Metab. 2010;99(Suppl 1):S86-9.

3. Blau N, Hennermann JB, Langenbeck U, Lichter-Konecki U. Diagnosis, classification, and genetics of phenylketonuria and tetrahydrobiopterin (BH4) deficiencies. Mol Genet Metab. 2011;104(Suppl):S2-9.

4. Loeber JG. Neonatal screening in Europe; the situation in 2004. J Inherit Metab Dis. 2007;30(4):430-8.

5. Enns GM, Koch R, Brumm V, Blakely E, Suter R, Jurecki E. Suboptimal outcomes in patients with PKU treated early with diet alone: revisiting the evidence. Mol Genet Metab. 2010;101(2-3):99-109.

6. Ahring K, Belanger-Quintana A, Dokoupil K, Gokmen Ozel H, Lammardo AM, MacDonald A, et al. Dietary management practices in phenylketonuria across European centres. Clin Nutr. 2009;28(3):231-6.

7. Blau N, Belanger-Quintana A, Demirkol M, Feillet F, Giovannini M, MacDonald A, et al. Management of phenylketonuria in Europe: survey results from 19 countries. Mol Genet Metab. 2010;99(2):109-15.

8. van Spronsen FJ, Ahring KK, Gizewska M. PKU-what is daily practice in various centres in Europe? Data from a questionnaire by the scientific advisory committee of the European Society of Phenylketonuria and Allied Disorders. J Inherit Metab Dis. 2009;32(1):58-64.

9. van Spronsen FJ. Phenylketonuria management from an European perspective: a commentary. Mol Genet Metab. 2010;100(2):107-10.
10. van Spronsen FJ, Burgard P. The truth of treating patients with phenylketonuria after childhood: the need for a new guideline. J Inherit Metab Dis. 2008;31(6):673-9.

11. Hagedorn TS, van Berkel P, Hammerschmidt G, Lhotakova M, Saludes RP Requirements for a minimum standard of care for phenylketonuria: the patients' perspective. Orphanet J Rare Dis. 2013;8:191.

12. Weingarten $\mathrm{S}$. Translating practice guidelines into patient care: guidelines at the bedside. Chest. 2000;118(2 Suppl):4S-7S.

13. Cabana MD, Kiyoshi-Teo H. The broader picture on guideline adherence. JPEN J Parenter Enteral Nutr. 2010;34(6):593-4.

14. Haberle J, Boddaert N, Burlina A, Chakrapani A, Dixon M, Huemer M, et al. Suggested guidelines for the diagnosis and management of urea cycle disorders. Orphanet J Rare Dis. 2012;7:32.

15. van Spronsen FJ, van Wegberg AM, Ahring K, Belanger-Quintana A, Blau N, Bosch AM, et al. Key European guidelines for the diagnosis and management of patients with phenylketonuria. Lancet Diabetes Endocrinol. 2017.

16. Geelhoed EA, Lewis B, Hounsome D, O'Leary P. Economic evaluation of neonatal screening for phenylketonuria and congenital hypothyroidism. J Paediatr Child Health. 2005:41(11):575-9.

17. Lord J, Thomason MJ, Littlejohns P, Chalmers RA, Bain MD, Addison GM, et al. Secondary analysis of economic data: a review of cost-benefit studies of neonatal screening for phenylketonuria. J Epidemiol Community Health. 1999:53(3):179-86.

18. Thomason MJ, Lord J, Bain MD, Chalmers RA, Littlejohns P, Addison GM, et al. A systematic review of evidence for the appropriateness of neonatal screening programmes for inborn errors of metabolism. J Public Health Med. 1998;20(3):331-43.

19. Dhondt JL. Strategy for the screening of tetrahydrobiopterin deficiency among hyperphenylalaninaemic patients: 15-years experience. J Inherit Metab Dis. 1991:14(2):117-27.

20. Opladen T, Hoffmann GF, Blau N. An international survey of patients with tetrahydrobiopterin deficiencies presenting with hyperphenylalaninaemia. J Inherit Metab Dis. 2012;35(6):963-73.

21. Blau N, Ichinose $H$, Nagatsu T, Heizmann CW, Zacchello F, Burlina AB. A missense mutation in a patient with guanosine triphosphate cyclohydrolase I deficiency missed in the newborn screening program. J Pediatr. 1995; 126(3):401-5.

22. Bonafe L, Thony B, Penzien JM, Czarnecki B, Blau N. Mutations in the sepiapterin reductase gene cause a novel tetrahydrobiopterin-dependent monoamine-neurotransmitter deficiency without hyperphenylalaninemia. Am J Hum Genet. 2001;69(2):269-77.

23. Niederwieser A, Ponzone A, Curtius HC. Differential diagnosis of tetrahydrobiopterin deficiency. J Inherit Metab Dis. 1985;8(Suppl 1):34-8.

24. Opladen T, Abu Seda B, Rassi A, Thony B, Hoffmann GF, Blau N. Diagnosis of tetrahydrobiopterin deficiency using filter paper blood spots: further development of the method and 5 years experience. J Inherit Metab Dis. 2011;34(3):819-26.

25. Cao YY, Qu YJ, Song F, Zhang T, Bai JL, Jin YW, et al. Fast clinical molecular diagnosis of hyperphenylalaninemia using next-generation sequencingbased on a custom AmpliSeq panel and ion torrent PGM sequencing. Mol Genet Metab. 2014;113(4):261-6.

26. Trujillano D, Perez B, Gonzalez J, Tornador C, Navarrete R, Escaramis G, et al. Accurate molecular diagnosis of phenylketonuria and tetrahydrobiopterindeficient hyperphenylalaninemias using high-throughput targeted sequencing. Eur J Hum Genet. 2014;22(4):528-34.

27. Simaite D, Kofent J, Gong M, Ruschendorf F, Jia S, Arn P, et al. Recessive mutations in PCBD1 cause a new type of early-onset diabetes. Diabetes. 2014;63(10):3557-64

28. Ferre S, de Baaij JH, Ferreira P, Germann R, de Klerk JB, Lavrijsen M, et al. Mutations in PCBD1 cause hypomagnesemia and renal magnesium wasting J Am Soc Nephrol. 2014;25(3):574-86.

29. Danecka MK, Woidy M, Zschocke J, Feillet F, Muntau AC, Gersting SW. Mapping the functional landscape of frequent phenylalanine hydroxylase (PAH) genotypes promotes personalised medicine in phenylketonuria. J Med Genet. 2015;52(3):175-85.

30. Guldberg P, Rey F, Zschocke J, Romano V, Francois B, Michiels L, et al. A European multicenter study of phenylalanine hydroxylase deficiency: classification of 105 mutations and a general system for genotype-based prediction of metabolic phenotype. Am J Hum Genet. 1998;63(1):71-9.

31. Pey AL, Stricher F, Serrano L, Martinez A. Predicted effects of missense mutations on native-state stability account for phenotypic outcome in 
phenylketonuria, a paradigm of misfolding diseases. Am J Hum Genet. 2007; 81(5):1006-24.

32. Wettstein S, Underhaug J, Perez B, Marsden BD, Yue WW, Martinez A, et al. Linking genotypes database with locus-specific database and genotypephenotype correlation in phenylketonuria. Eur J Hum Genet. 2015;23(3):302-9.

33. Staudigl M, Gersting SW, Danecka MK, Messing DD, Woidy M, Pinkas D, et al. The interplay between genotype, metabolic state and cofactor treatment governs phenylalanine hydroxylase function and drug response. Hum Mol Genet. 2011;20(13):2628-41.

34. Zurfluh MR, Zschocke J, Lindner M, Feillet F, Chery C, Burlina A, et al. Molecular genetics of tetrahydrobiopterin-responsive phenylalanine hydroxylase deficiency. Hum Mutat. 2008;29(1):167-75.

35. Anjema K, van Rijn M, Hofstede FC, Bosch AM, Hollak CE, Rubio-Gozalbo E, et al. Tetrahydrobiopterin responsiveness in phenylketonuria: prediction with the 48-hour loading test and genotype. Orphanet J Rare Dis. 2013;8: 103.

36. Trefz FK, Scheible D, Gotz H, Frauendienst-Egger G. Significance of genotype in tetrahydrobiopterin-responsive phenylketonuria. J Inherit Metab Dis. 2009; 32(1):22-6.

37. Blaskovics ME, Schaeffler GE, Hack S. Phenylalaninaemia. Differential diagnosis. Arch Dis Child. 1974;49(11):835-43.

38. Guttler F. Hyperphenylalaninemia: diagnosis and classification of the various types of phenylalanine hydroxylase deficiency in childhood. Acta Paediatr Scand Suppl. 1980;280:1-80.

39. Campistol J, Gassio R, Artuch R, Vilaseca MA, Unit PKUF-u. Neurocognitive function in mild hyperphenylalaninemia. Dev Med Child Neurol. 2011;53(5): 405-8.

40. van Spronsen FJ, van Rijn M, Dorgelo B, Hoeksma M, Bosch AM, Mulder MF, et al. Phenylalanine tolerance can already reliably be assessed at the age of 2 years in patients with PKU. J Inherit Metab Dis. 2009:32(1):27-31.

41. Guttler F, Guldberg P. The influence of mutations of enzyme activity and phenylalanine tolerance in phenylalanine hydroxylase deficiency. Eur Pediatr. 1996;155(Suppl 1):S6-10.

42. Smith I, Beasley MG, Ades AE. Intelligence and quality of dietary treatment in phenylketonuria. Arch Dis Child. 1990;65(5):472-8.

43. Gassio R, Artuch R, Vilaseca MA, Fuste E, Boix C, Sans A, et al. Cognitive functions in classic phenylketonuria and mild hyperphenylalaninaemia: experience in a paediatric population. Dev Med Child Neurol. 2005;47(7): 443-8.

44. Berlin CM LH, Hanley WB. Delayed increase in blood phenylalanine concentration in phenylketonuric children initially classified as mild hyperphenylalaninemia. 1995;4:35-9.

45. Schulze A, Mayatepek E, Hoffmann GF. Evaluation of 6-year application of the enzymatic colorimetric phenylalanine assay in the setting of neonatal screening for phenylketonuria. Clin Chim Acta. 2002;317(1-2):27-37.

46. van Spronsen FJ. Mild hyperphenylalaninemia: to treat or not to treat. J Inherit Metab Dis. 2011;34(3):651-6.

47. Costello PM, Beasley MG, Tillotson SL, Smith I. Intelligence in mild atypical phenylketonuria. Eur J Pediatr. 1994;153(4):260-3.

48. Diamond A, Prevor MB, Callender G, Druin DP. Prefrontal cortex cognitive deficits in children treated early and continuously for PKU. Monogr Soc Res Child Dev. 1997;62((4):i-v):1-208.

49. Weglage J, Pietsch M, Feldmann R, Koch HG, Zschocke J, Hoffmann G, et al. Normal clinical outcome in untreated subjects with mild hyperphenylalaninemia. Pediatr Res. 2001;49(4):532-6.

50. Smith ML, Saltzman J, Klim P, Hanley WB, Feigenbaum A, Clarke JT. Neuropsychological function in mild hyperphenylalaninemia. Am J Ment Retard. 2000;105(2):69-80.

51. Widaman KF. Phenylketonuria in children and mothers: genes, environments, behavior. Curr Dir Psychol Sci. 2009;18(1):48,

52. Fonnesbeck CJ, McPheeters ML, Krishnaswami S, Lindegren ML, Reimschisel T. Estimating the probability of IQ impairment from blood phenylalanine for phenylketonuria patients: a hierarchical meta-analysis. J Inherit Metab Dis. 2013;36(5):757-66.

53. Waisbren SE, Noel K, Fahrbach K, Cella C, Frame D, Dorenbaum A, et al. Phenylalanine blood levels and clinical outcomes in phenylketonuria: a systematic literature review and meta-analysis. Mol Genet Metab. 2007;92(12):63-70.

54. Azen CG, Koch R, Friedman EG, Berlow S, Coldwell J, Krause W, et al. Intellectual development in 12-year-old children treated for phenylketonuria. Am J Dis Child. 1991;145(1):35-9.
55. Cabalska B, Duczynska N, Borzymowska J, Zorska K, Koslacz-Folga A, Bozkowa K. Termination of dietary treatment in phenylketonuria. Eur J Pediatr. 1977;126(4):253-62.

56. Bosch AM, Tybout W, van Spronsen FJ, de Valk HW, Wijburg FA, Grootenhuis MA. The course of life and quality of life of early and continuously treated Dutch patients with phenylketonuria. J Inherit Metab Dis. 2007;30(1):29-34.

57. Regnault A, Burlina A, Cunningham A, Bettiol E, Moreau-Stucker F, Benmedjahed K, et al. Development and psychometric validation of measures to assess the impact of phenylketonuria and its dietary treatment on patients' and parents' quality of life: the phenylketonuria - quality of life (PKU-QOL) questionnaires. Orphanet J Rare Dis. 2015;10(1):59.

58. Bosch AM, Burlina A, Cunningham A, Bettiol E, Moreau-Stucker F, Benmedjahed $\mathrm{K}$, et al. Assessment of the impact of phenylketonuria and its treatment on quality of life of patients and parents from seven European countries. Orphanet J Rare Dis. 2015;10(1):80.

59. Albrecht J, Garbade SF, Burgard P. Neuropsychological speed tests and blood phenylalanine levels in patients with phenylketonuria: a metaanalysis. Neurosci Biobehav Rev. 2009:33(3):414-21.

60. Weglage J, Fromm J, van Teeffelen-Heithoff A, Moller HE, Koletzko B, Marquardt T, et al. Neurocognitive functioning in adults with phenylketonuria: results of a long term study. Mol Genet Metab. 2013;110 Suppl:S44-S48.

61. Bik-Multanowski M, Didycz B, Mozrzymas R, Nowacka M, Kaluzny L, Cichy W, et al. Quality of life in noncompliant adults with phenylketonuria after resumption of the diet. J Inherit Metab Dis. 2008;31(Suppl 2):S415-8.

62. Simon E, Schwarz M, Roos J, Dragano N, Geraedts M, Siegrist J, et al. Evaluation of quality of life and description of the sociodemographic state in adolescent and young adult patients with phenylketonuria (PKU). Health Qual Life Outcomes. 2008;6:25.

63. Dawson C, Murphy E, Maritz C, Chan H, Ellerton C, Carpenter RH, et al. Dietary treatment of phenylketonuria: the effect of phenylalanine on reaction time. J Inherit Metab Dis. 2011;34(2):449-54.

64. Channon S, Goodman G, Zlotowitz S, Mockler C, Lee PJ. Effects of dietary management of phenylketonuria on long-term cognitive outcome. Arch Dis Child. 2007;92(3):213-8.

65. Koch R, Burton B, Hoganson G, Peterson R, Rhead W, Rouse B, et al. Phenylketonuria in adulthood: a collaborative study. J Inherit Metab Dis. 2002;25(5):333-46

66. Daelman L, Sedel F, Tourbah A. Progressive neuropsychiatric manifestations of phenylketonuria in adulthood. Rev Neurol. 2014;170(4):280-7.

67. Anwar MS, Waddell B, O'Riordan J. Neurological improvement following reinstitution of a low phenylalanine diet after 20 years in established phenylketonuria. BMJ Case Rep. 2013;2013.

68. Rubin S, Piffer AL, Rougier MB, Delyfer MN, Korobelnik JF, Redonnet-Vernhet I, et al. Sight-threatening phenylketonuric encephalopathy in a young adult, reversed by diet. JIMD Rep. 2013;10:83-5.

69. Schmidt E, Burgard P, Rupp A. Effects of concurrent phenylalanine levels on sustained attention and calculation speed in patients treated early for phenylketonuria. Eur J Pediatr. 1996;155(Suppl 1):S82-6.

70. ten Hoedt AE, de Sonneville LM, Francois B, ter Horst NM, Janssen MC, Rubio-Gozalbo ME, et al. High phenylalanine levels directly affect mood and sustained attention in adults with phenylketonuria: a randomised, doubleblind, placebo-controlled, crossover trial. J Inherit Metab Dis. 2011;34(1):16571.

71. Gassio R, Campistol J, Vilaseca MA, Lambruschini N, Cambra FJ, Fuste E. Do adult patients with phenylketonuria improve their quality of life after introduction/resumption of a phenylalanine-restricted diet? Acta Paediatr. 2003:92(12):1474-8.

72. van Spronsen FJ, Huijbregts SC, Bosch AM, Leuzzi V. Cognitive, neurophysiological, neurological and psychosocial outcomes in earlytreated PKU-patients: a start toward standardized outcome measurement across development. Mol Genet Metab. 2011;104 Suppl:S45-51.

73. Perez-Duenas B, Valls-Sole J, Fernandez-Alvarez E, Conill J, Vilaseca MA, Artuch $\mathrm{R}$, et al. Characterization of tremor in phenylketonuric patients. J Neurol. 2005;252(11):1328-34.

74. Trefz F, Maillot F, Motzfeldt K, Schwarz M. Adult phenylketonuria outcome and management. Mol Genet Metab. 2011;104 Suppl:S26-30.

75. Rohde C, von Teeffelen-Heithoff A, Thiele AG, Arelin M, Mutze U, Kiener C, et al. PKU patients on a relaxed diet may be at risk for micronutrient deficiencies. Eur J Clin Nutr. 2014;68(1):119-24. 
76. Gokmen H, Ozel KA, Bélanger-Quintana A. Overweight and obesity in PKU: the results from 8 centres in Europe and Turkey. Mol Genet Metab Rep. 2014;1:483-6.

77. Robertson LV, McStravick N, Ripley S, Weetch E, Donald S, Adam S, et al. Body mass index in adult patients with diet-treated phenylketonuria. J Hum Nutr Diet. 2013;26(Suppl 1):1-6.

78. MacDonald A, Ahring K, Almeida MF, Belanger-Quintana A, Blau N, Burlina $A$, et al. The challenges of managing coexistent disorders with phenylketonuria: 30 cases. Mol Genet Metab. 2015.

79. Demirdas S, Coakley KE, Bisschop PH, Hollak CE, Bosch AM, Singh RH. Bone health in phenylketonuria: a systematic review and meta-analysis. Orphanet J Rare Dis. 2015;10:17.

80. Donati A, Vincenzi C, Tosti A. Acute hair loss in phenylketonuria. J Eur Acad Dermatol Venereol. 2009;23(5):613-5.

81. Pode-Shakked B, Shemer-Meiri L, Harmelin A, Stettner N, Brenner O, Abraham S, et al. Man made disease: clinical manifestations of low phenylalanine levels in an inadequately treated phenylketonuria patient and mouse study. Mol Genet Metab. 2013;110 Suppl:S66-70.

82. van Spronsen FJ, van Rijn M, van Dijk T, Smit GP, Reijngoud DJ, Berger R, et al. Plasma phenylalanine and tyrosine responses to different nutritional conditions (fasting/postprandial) in patients with phenylketonuria: effect of sample timing. Pediatrics. 1993;92(4):570-3.

83. De Silva V, Oldham CD, May SW. L-phenylalanine concentration in blood of phenylketonuria patients: a modified enzyme colorimetric assay compared with amino acid analysis, tandem mass spectrometry, and HPLC methods. Clin Chem Lab Med. 2010;48(9):1271-9.

84. Gregory CO, Yu C, Singh RH. Blood phenylalanine monitoring for dietary compliance among patients with phenylketonuria: comparison of methods. Genet Med. 2007;9(11):761-5.

85. Wibrand F. A microplate-based enzymatic assay for the simultaneous determination of phenylalanine and tyrosine in serum. Clin Chim Acta. 2004;347(1-2):89-96

86. Mo XM, Li Y, Tang AG, Ren YP. Simultaneous determination of phenylalanine and tyrosine in peripheral capillary blood by HPLC with ultraviolet detection. Clin Biochem. 2013;46(12):1074-8.

87. Groselj U, Murko S, Zerjav Tansek M, Kovac J, Trampus Bakija A, Repic Lampret $B$, et al. Comparison of tandem mass spectrometry and amino acid analyzer for phenylalanine and tyrosine monitoring-implications for clinical management of patients with hyperphenylalaninemia. Clin Biochem. 2015; 48(1-2):14-8.

88. Holub M, Tuschl K, Ratschmann R, Strnadova KA, Muhl A, Heinze G, et al. Influence of hematocrit and localisation of punch in dried blood spots on levels of amino acids and acylcarnitines measured by tandem mass spectrometry. Clin Chim Acta. 2006;373(1-2):27-31.

89. Kand'ar R, Zakova P. Determination of phenylalanine and tyrosine in plasma and dried blood samples using HPLC with fluorescence detection. J Chromatogr B Anal Technol Biomed Life Sci. 2009;877(30):3926-9.

90. Lawson AJ, Bernstone L, Hall SK. Newborn screening blood spot analysis in the UK: influence of spot size, punch location and haematocrit. J Med Screen. 2016;23(1):7-16.

91. Prinsen HC, Holwerda-Loof NE, de Sain-van der Velden MG, Visser G, Verhoeven-Duif NM. Reliable analysis of phenylalanine and tyrosine in a minimal volume of blood. Clin Biochem. 2013;46(13-14):1272-5.

92. Leuzzi V, Pansini M, Sechi E, Chiarotti F, Carducci C, Levi G, et al. Executive function impairment in early-treated PKU subjects with normal mental development. J Inherit Metab Dis. 2004;27(2):115-25.

93. Huijbregts SC, de Sonneville LM, van Spronsen FJ, Licht R, Sergeant JA. The neuropsychological profile of early and continuously treated phenylketonuria: orienting, vigilance, and maintenance versus manipulationfunctions of working memory. Neurosci Biobehav Rev. 2002;26(6):697-712.

94. Jahja R, Huijbregts SC, de Sonneville LM, van der Meere JJ, van Spronsen FJ. Neurocognitive evidence for revision of treatment targets and guidelines for phenylketonuria. J Pediatr. 2014;164(4):895-9. e2

95. Moyle JJ, Fox AM, Arthur M, Bynevelt M, Burnett JR. Meta-analysis of neuropsychological symptoms of adolescents and adults with PKU. Neuropsychol Rev. 2007;17(2):91-101.

96. Hoeksma M, Reijngoud DJ, Pruim J, de Valk HW, Paans AM, van Spronsen FJ. Phenylketonuria: high plasma phenylalanine decreases cerebral protein synthesis. Mol Genet Metab. 2009;96(4):177-82.

97. Bick U, Ullrich K, Stober U, Moller H, Schuierer G, Ludolph AC, et al. White matter abnormalities in patients with treated hyperphenylalaninaemia: magnetic resonance relaxometry and proton spectroscopy findings. Eur J Pediatr. 1993;152(12):1012-20.

98. Kono K, Okano Y, Nakayama K, Hase Y, Minamikawa S, Ozawa N, et al. Diffusion-weighted MR imaging in patients with phenylketonuria: relationship between serum phenylalanine levels and $A D C$ values in cerebral white matter. Radiology. 2005;236(2):630-6.

99. Lou HC, Toft PB, Andresen J, Mikkelsen I, Olsen B, Guttler F, et al. An occipito-temporal syndrome in adolescents with optimally controlled hyperphenylalaninaemia. J Inherit Metab Dis. 1992;15(5):687-95.

100. Manara R, Burlina AP, Citton V, Ermani M, Vespignani F, Carollo C, et al. Brain MRI diffusion-weighted imaging in patients with classical phenylketonuria. Neuroradiology. 2009;51(12):803-12.

101. Sanayama Y, Nagasaka H, Takayanagi M, Ohura T, Sakamoto O, Ito T, et al. Experimental evidence that phenylalanine is strongly associated to oxidative stress in adolescents and adults with phenylketonuria. Mol Genet Metab. 2011;103(3):220-5

102. Vockley J, Andersson HC, Antshel KM, Braverman NE, Burton BK, Frazier DM, et al. Phenylalanine hydroxylase deficiency: diagnosis and management guideline. Genet Med. 2014;16(2):188-200.

103. MacDonald A, Rylance G, Hall SK, Asplin D, Booth IW. Factors affecting the variation in plasma phenylalanine in patients with phenylketonuria on diet. Arch Dis Child. 1996;74(5):412-7.

104. van Spronsen FJ, van Dijk T, Smit GP, van Rijn M, Reijngoud DJ, Berger R, et al. Phenylketonuria: plasma phenylalanine responses to different distributions of the daily phenylalanine allowance over the day. Pediatrics. 1996;97(6 Pt 1):839-44.

105. Anastasoaie V, Kurzius L, Forbes $P$, Waisbren S. Stability of blood phenylalanine levels and IQ in children with phenylketonuria. Mol Genet Metab. 2008;95(1-2):17-20.

106. Hood A, Grange DK, Christ SE, Steiner R, White DA. Variability in phenylalanine control predicts IQ and executive abilities in children with phenylketonuria. Mol Genet Metab. 2014;111(4):445-51.

107. Vilaseca MA, Lambruschini N, Gomez-Lopez L, Gutierrez A, Fuste E, Gassio R, et al. Quality of dietary control in phenylketonuric patients and its relationship with general intelligence. Nutr Hosp. 2010;25(1):60-6.

108. Viau KS, Wengreen HJ, Ernst SL, Cantor NL, Furtado LV, Longo N. Correlation of age-specific phenylalanine levels with intellectual outcome in patients with phenylketonuria. J Inherit Metab Dis. 2011:34(4):963-71.

109. Cleary M, Trefz F, Muntau AC, Feillet F, van Spronsen FJ, Burlina A, et al. Fluctuations in phenylalanine concentrations in phenylketonuria: a review of possible relationships with outcomes. Mol Genet Metab. 2013;110(4):418-23.

110. Sharman R, Sullivan K, Young R, McGill J. Biochemical markers associated with executive function in adolescents with early and continuously treated phenylketonuria. Clin Genet. 2009;75(2):169-74.

111. Sharman R, Sullivan $K$, Young R, McGill J. A preliminary investigation of the role of the phenylalanine:tyrosine ratio in children with early and continuously treated phenylketonuria: toward identification of "safe" levels. Dev Neuropsychol. 2010;35(1):57-65.

112. Sharman R, Sullivan K, Young RM, McGill J. Depressive symptoms in adolescents with early and continuously treated phenylketonuria: associations with phenylalanine and tyrosine levels. Gene. 2012;504(2):28891.

113. Luciana M, Sullivan J, Nelson CA. Associations between phenylalanine-totyrosine ratios and performance on tests of neuropsychological function in adolescents treated early and continuously for phenylketonuria. Child Dev. 2001;72(6):1637-52.

114. van Spronsen FJ, van Dijk T, Smit GP, van Rijn M, Reijngoud DJ, Berger $R$, et al. Large daily fluctuations in plasma tyrosine in treated patients with phenylketonuria. Am J Clin Nutr. 1996;64(6):916-21.

115. Crone MR, van Spronsen FJ, Oudshoorn K, Bekhof J, van Rijn G, Verkerk PH. Behavioural factors related to metabolic control in patients with phenylketonuria. J Inherit Metab Dis. 2005;28(5):627-37.

116. Trefz FK, van Spronsen FJ, MacDonald A, Feillet F, Muntau AC, BelangerQuintana A, et al. Management of adult patients with phenylketonuria: survey results from 24 countries. Eur J Pediatr. 2015;174(1):119-27.

117. Mutze U, Roth A, Weigel JF, Beblo S, Baerwald CG, Buhrdel P, et al. Transition of young adults with phenylketonuria from pediatric to adult care. J Inherit Metab Dis. 2011:34(3):701-9.

118. Evans S, Daly A, MacDonald J, Preece MA, Santra S, Vijay S, et al. The micronutrient status of patients with phenylketonuria on dietary treatment: an ongoing challenge. Ann Nutr Metab. 2014;65(1):42-8. 
119. Lammardo AM, Robert M, Rocha JC, van Rijn M, Ahring K, BelangerQuintana A, et al. Main issues in micronutrient supplementation in phenylketonuria. Mol Genet Metab. 2013;110 Suppl:S1-5.

120. Robert M, Rocha JC, van Rijn M, Ahring K, Belanger-Quintana A, MacDonald A, et al. Micronutrient status in phenylketonuria. Mol Genet Metab. 2013;110 Suppl:S6-17.

121. Hanley WB, Feigenbaum A, Clarke JT, Schoonheyt W, Austin V. Vitamin B12 deficiency in adolescents and young adults with phenylketonuria. Lancet. 1993;342(8877):997.

122. Lee P, Smith I, Piesowicz A, Brenton D. Spastic paraparesis after anaesthesia. Lancet. 1999;353(9152):554

123. Barretto JR, Silva LR, Leite ME, Boa-Sorte N, Pimentel H, Purificacao AC, et al. Poor zinc and selenium status in phenylketonuric children and adolescents in Brazil. Nutr Res. 2008;28(3):208-11.

124. Prochazkova D, Jarkovsky J, Vinohradska H, Konecna P, Machacova L, Dolezel Z. Controlled diet in phenylketonuria and hyperphenylalaninemia may cause serum selenium deficiency in adult patients: the Czech experience. Biol Trace Elem Res. 2013;154(2):178-84.

125. Arnold GL, Kirby R, Preston C, Blakely E. Iron and protein sufficiency and red cell indices in phenylketonuria. J Am Coll Nutr. 2001;20(1):65-70.

126. Bodley JL, Austin VJ, Hanley WB, Clarke JT, Zlotkin S. Low iron stores in infants and children with treated phenylketonuria: a population at risk for iron-deficiency anaemia and associated cognitive deficits. Eur J Pediatr. 1993;152(2):140-3.

127. Wiig I, Motzfeldt K, Loken EB, Kase BF. Nutritional consequences of adhering to a low phenylalanine diet for late-treated adults with PKU : low Phe diet for adults with PKU. JIMD Rep. 2013;7:109-16.

128. Miranda da Cruz BD, Seidler H, Widhalm K. Iron status and iron supplementation in children with classical phenylketonuria. J Am Coll Nutr. 1993;12(5):531-6.

129. Vugteveen I, Hoeksma M, Monsen AL, Fokkema MR, Reijngoud DJ, van Rijn $M$, et al. Serum vitamin B12 concentrations within reference values do not exclude functional vitamin B12 deficiency in PKU patients of various ages. Mol Genet Metab. 2011;102(1):13-7.

130. Stolen LH, Lilje R, Jorgensen JV, Bliksrud YT, Almaas R. High dietary folic acid and high plasma folate in children and adults with phenylketonuria. JIMD Rep. 2014;13:83-90.

131. Crabtree NJ, Arabi A, Bachrach LK, Fewtrell M, El-Hajj Fuleihan G, Kecskemethy $\mathrm{HH}$, et al. Dual-energy $\mathrm{X}$-ray absorptiometry interpretation and reporting in children and adolescents: the revised 2013 ISCD Pediatric official positions. J Clin Densitom. 2014;17(2):225-42.

132. Kanis JA, McCloskey EV, Johansson H, Cooper C, Rizzoli R, Reginster JY, et al. European guidance for the diagnosis and management of osteoporosis in postmenopausal women. Osteoporos Int. 2013;24(1):23-57.

133. Hansen KE, Ney D. A systematic review of bone mineral density and fractures in phenylketonuria. J Inherit Metab Dis. 2014:37(6):875-80.

134. Greeves LG, Carson DJ, Magee A, Patterson CC. Fractures and phenylketonuria. Acta Paediatr. 1997;86(3):242-4.

135. Perez-Duenas B, Cambra FJ, Vilaseca MA, Lambruschini N, Campistol J Camacho JA. New approach to osteopenia in phenylketonuric patients. Acta Paediatr. 2002:91(8):899-904.

136. Zeman J, Bayer M, Stepan J. Bone mineral density in patients with phenylketonuria. Acta Paediatr. 1999;88(12):1348-51.

137. Miras A, Boveda MD, Leis MR, Mera A, Aldamiz-Echevarria L, FernandezLorenzo JR, et al. Risk factors for developing mineral bone disease in phenylketonuric patients. Mol Genet Metab. 2013;108(3):149-54.

138. Nagasaka H, Tsukahara $H$, Takatani T, Sanayama Y, Takayanagi M, Ohura T, et al. Cross-sectional study of bone metabolism with nutrition in adult classical phenylketonuric patients diagnosed by neonatal screening. J Bone Miner Metab. 2011;29(6):737-43.

139. Modan-Moses D, Vered I, Schwartz G, Anikster Y, Abraham S, Segev R, et al. Peak bone mass in patients with phenylketonuria. J Inherit Metab Dis. 2007; 30(2):202-8

140. Porta F, Mussa A, Zanin A, Greggio NA, Burlina A, Spada M. Impact of metabolic control on bone quality in phenylketonuria and mild hyperphenylalaninemia. J Pediatr Gastroenterol Nutr. 2011;52(3):345-50.

141. Adamczyk P, Morawiec-Knysak A, Pludowski P, Banaszak B, Karpe J, Pluskiewicz W. Bone metabolism and the muscle-bone relationship in children, adolescents and young adults with phenylketonuria. J Bone Miner Metab. 2011:29(2):236-44.

142. Mendes AB, Martins FF, Cruz WM, da Silva LE, Abadesso CB, Boaventura GT. Bone development in children and adolescents with PKU. J Inherit Metab Dis. 2012;35(3):425-30.
143. Solverson P, Murali SG, Litscher SJ, Blank RD, Ney DM. Low bone strength is a manifestation of phenylketonuria in mice and is attenuated by a glycomacropeptide diet. PLoS One. 2012;7(9):e45165.

144. Anderson PJ, Leuzzi V. White matter pathology in phenylketonuria. Mol Genet Metab. 2010;99(Suppl 1):S3-9.

145. Camp KM, Parisi MA, Acosta PB, Berry GT, Bilder DA, Blau N, et al. Phenylketonuria scientific review conference: state of the science and future research needs. Mol Genet Metab. 2014;112(2):87-122.

146. Peng H, Peck D, White DA, Christ SE. Tract-based evaluation of white matter damage in individuals with early-treated phenylketonuria. J Inherit Metab Dis. 2014;37(2):237-43.

147. Antenor-Dorsey JA, Hershey T, Rutlin J, Shimony JS, McKinstry RC, Grange DK, et al. White matter integrity and executive abilities in individuals with phenylketonuria. Mol Genet Metab. 2013;109(2):125-31.

148. Cleary MA, Walter JH, Wraith JE, Jenkins JP, Alani SM, Tyler K, et al. Magnetic resonance imaging of the brain in phenylketonuria. Lancet. 1994;344(8915): 87-90.

149. Hood A, Antenor-Dorsey JA, Rutlin J, Hershey T, Shimony JS, McKinstry RC, et al. Prolonged exposure to high and variable phenylalanine levels over the lifetime predicts brain white matter integrity in children with phenylketonuria. Mol Genet Metab. 2015;114(1):19-24.

150. Leuzzi V, Tosetti M, Montanaro D, Carducci C, Artiola C, Carducci C, et al. The pathogenesis of the white matter abnormalities in phenylketonuria. A multimodal 3.0 tesla MRI and magnetic resonance spectroscopy (1H MRS) study. J Inherit Metab Dis. 2007;30(2):209-16.

151. Pietz J, Kreis R, Schmidt H, Meyding-Lamade UK, Rupp A, Boesch C. Phenylketonuria: findings at MR imaging and localized in vivo $\mathrm{H}-1 \mathrm{MR}$ spectroscopy of the brain in patients with early treatment. Radiology. 1996; 201(2):413-20

152. Thompson AJ, Tillotson S, Smith I, Kendall B, Moore SG, Brenton DP. Brain $\mathrm{MRI}$ changes in phenylketonuria. Associations with dietary status. Brain. 1993;116(Pt 4):811-21.

153. White DA, Connor LT, Nardos B, Shimony JS, Archer R, Snyder AZ, et al. Age-related decline in the microstructural integrity of white matter in children with early- and continuously-treated PKU: a DTI study of the corpus callosum. Mol Genet Metab. 2010;99(Suppl 1):S41-6.

154. Cleary MA, Walter JH, Wraith JE, White F, Tyler K, Jenkins JP. Magnetic resonance imaging in phenylketonuria: reversal of cerebral white matter change. J Pediatr. 1995;127(2):251-5.

155. White DA, Antenor-Dorsey JA, Grange DK, Hershey T, Rutlin J, Shimony JS, et al. White matter integrity and executive abilities following treatment with tetrahydrobiopterin $(\mathrm{BH} 4)$ in individuals with phenylketonuria. Mol Genet Metab. 2013:110(3):213-7.

156. Battistini S, De Stefano N, Parlanti S, Federico A. Unexpected white matter changes in an early treated PKU case and improvement after dietary treatment. Funct Neurol. 1991;6(2):177-80.

157. Thompson AJ, Smith I, Brenton D, Youl BD, Rylance G, Davidson DC, et al. Neurological deterioration in young adults with phenylketonuria. Lancet. 1990:336(8715):602-5.

158. Gassio R, Fuste E, Lopez-Sala A, Artuch R, Vilaseca MA, Campistol J. School performance in early and continuously treated phenylketonuria. Pediatr Neurol. 2005;33(4):267-71.

159. Anjema K, van Rijn M, Verkerk PH, Burgerhof JG, Heiner-Fokkema MR, van Spronsen FJ. PKU: high plasma phenylalanine concentrations are associated with increased prevalence of mood swings. Mol Genet Metab. 2011;104(3): $231-4$.

160. Janzen D, Nguyen M. Beyond executive function: non-executive cognitive abilities in individuals with PKU. Mol Genet Metab. 2010;99(Suppl 1):S47-51.

161. Christ SE, Huijbregts SC, de Sonneville LM, White DA. Executive function in early-treated phenylketonuria: profile and underlying mechanisms. Mol Genet Metab. 2010;99(Suppl 1):S22-32.

162. Liemburg GB, Jahja R, van Spronsen FJ, de Sonneville LM, van der Meere JJ, Bosch AM, et al. Is BRIEF a useful instrument in day to day care of patients with phenylketonuria? Mol Genet Metab. 2015:114(3):425-30.

163. Landolt MA, Nuoffer JM, Steinmann B, Superti-Furga A. Quality of life and psychologic adjustment in children and adolescents with early treated phenylketonuria can be normal. J Pediatr. 2002;140(5):516-21.

164. Thimm E, Schmidt LE, Heldt K, Spiekerkoetter U. Health-related quality of life in children and adolescents with phenylketonuria: unimpaired HRQOL in patients but feared school failure in parents. J Inherit Metab Dis. 2013:36(5): $767-72$. 
165. Demirdas S, Maurice-Stam H, Boelen CC, Hofstede FC, Janssen MC, Langendonk JG, et al. Evaluation of quality of life in PKU before and after introducing tetrahydrobiopterin (BH4); a prospective multi-center cohort study. Mol Genet Metab. 2013;110 Suppl:S49-56.

166. Cotugno G, Nicolo R, Cappelletti S, Goffredo BM, Dionisi Vici C, Di Ciommo $\checkmark$. Adherence to diet and quality of life in patients with phenylketonuria. Acta Paediatr. 2011;100(8):1144-9.

167. Engelen V, Detmar S, Koopman H, Maurice-Stam H, Caron H, Hoogerbrugge $P$, et al. Reporting health-related quality of life scores to physicians during routine follow-up visits of pediatric oncology patients: is it effective? Pediatr Blood Cancer. 2012;58(5):766-74.

168. Takeuchi EE, Keding A, Awad N, Hofmann U, Campbell LJ, Selby PJ, et al. Impact of patient-reported outcomes in oncology: a longitudinal analysis of patient-physician communication. J Clin Oncol. 2011;29(21):2910-7.

169. Velikova G, Booth L, Smith AB, Brown PM, Lynch P, Brown JM, et al. Measuring quality of life in routine oncology practice improves communication and patient well-being: a randomized controlled trial. J Clin Oncol. 2004;22(4):714-24.

170. de Wit M, Delemarre-van de Waal HA, Bokma JA, Haasnoot K, Houdijk MC, Gemke RJ, et al. Monitoring and discussing health-related quality of life in adolescents with type 1 diabetes improve psychosocial well-being: a randomized controlled trial. Diabetes Care. 2008;31(8):1521-6.

171. de Wit M, Delemarre-van de Waal HA, Bokma JA, Haasnoot K, Houdijk MC, Gemke RJ, et al. Follow-up results on monitoring and discussing healthrelated quality of life in adolescent diabetes care: benefits do not sustain in routine practice. Pediatr Diabetes. 2010;11(3):175-81.

172. Cappelletti S, Cotugno G, Goffredo BM, Nicolo R, Bernabei SM, Caviglia S, et al. Cognitive findings and behavior in children and adolescents with phenylketonuria. J Dev Behav Pediatr. 2013;34(6):392-8.

173. Hendrikx MM, van der Schot LW, Slijper FM, Huisman J, Kalverboer AF. Phenylketonuria and some aspects of emotional development. Eur J Pediatr. 1994;153(11):832-5.

174. Jusiene R, Kucinskas V. Familial variables as predictors of psychological maladjustment in Lithuanian children with phenylketonuria. Med Sci Monit. 2004;10(3):CR102-7.

175. Weglage J, Grenzebach M, Pietsch M, Feldmann R, Linnenbank R, Denecke $J$, et al. Behavioural and emotional problems in early-treated adolescents with phenylketonuria in comparison with diabetic patients and healthy controls. J Inherit Metab Dis. 2000;23(5):487-96.

176. Wu W, Sheng D, Shao J, Zhao Z. Mental and motor development and psychosocial adjustment of Chinese children with phenylketonuria. J Paediatr Child Health. 2011;47(7):441-7.

177. Jahja R, Huijbregts SC, de Sonneville LM, van der Meere JJ, Bosch AM, Hollak CE, et al. Mental health and social functioning in early treated Phenylketonuria: the PKU-COBESO study. Mol Genet Metab. 2013;110 Suppl:S57-61.

178. Smith I, Beasley MG, Wolff OH, Ades AE. Behavior disturbance in 8-year-old children with early treated phenylketonuria. Report from the MRC/DHSS Phenylketonuria register. J Pediatr. 1988;112(3):403-8.

179. Burgard P, Armbruster M, Schmidt E, Rupp A. Psychopathology of patients treated early for phenylketonuria: results of the German collaborative study of phenylketonuria. Acta Paediatr Suppl. 1994;407:108-10.

180. Sullivan JE. Emotional outcome of adolescents and young adults with early and continuously treated phenylketonuria. J Pediatr Psychol. 2001;26(8):477-84

181. Weglage J, Funders B, Ullrich K, Rupp A, Schmidt E. Psychosocial aspects in phenylketonuria. Eur J Pediatr. 1996:155(Suppl 1):S101-4.

182. Arnold GL, Vladutiu CJ, Orlowski CC, Blakely EM, DeLuca J. Prevalence of stimulant use for attentional dysfunction in children with phenylketonuria. J Inherit Metab Dis. 2004;27(2):137-43.

183. Burton B, Grant M, Feigenbaum A, Singh R, Hendren R, Siriwardena K, et al. A randomized, placebo-controlled, double-blind study of sapropterin to treat ADHD symptoms and executive function impairment in children and adults with sapropterin-responsive phenylketonuria. Mol Genet Metab. 2015; 114(3):415-24

184. Baieli S, Pavone L, Meli C, Fiumara A, Coleman M. Autism and phenylketonuria. J Autism Dev Disord. 2003;33(2):201-4.

185. Sirtori LR, Dutra-Filho CS, Fitarelli D, Sitta A, Haeser A, Barschak AG, et al. Oxidative stress in patients with phenylketonuria. Biochim Biophys Acta. 2005;1740(1):68-73.

186. Lombeck I, Jochum F, Terwolbeck K. Selenium status in infants and children with phenylketonuria and in maternal phenylketonuria. Eur J Pediatr. 1996; 155(Suppl 1):S140-4
187. Sitta A, Vanzin CS, Biancini GB, Manfredini V, de Oliveira AB, Wayhs CA, et al. Evidence that $\mathrm{L}$-carnitine and selenium supplementation reduces oxidative stress in phenylketonuric patients. Cell Mol Neurobiol. 2011;31(3):429-36.

188. Donlon JSC, Levy H, Scriver CR. Hyperphenylalaninemia: phenylalanine Hydroxylase deficiency. In: BA VD, Vogelstein B, Kinzler KW, Antonarakis SE, Ballabio A, Scriver CR, Sly WS, Bunz F, Gibson KM, Mitchell G, editors. The online metabolic and molecular bases of inherited disease. New York: The McGraw-Hill Companies; 2008. p. 1-14.

189. Macleod EL, Ney DM. Nutritional Management of Phenylketonuria. Ann Nestle. 2010;68(2):58-69.

190. MacLeod EL, Gleason ST, van Calcar SC, Ney DM. Reassessment of phenylalanine tolerance in adults with phenylketonuria is needed as body mass changes. Mol Genet Metab. 2009:98(4):331-7.

191. Rohde C, Mutze U, Weigel JF, Ceglarek U, Thiery J, Kiess W, et al. Unrestricted consumption of fruits and vegetables in phenylketonuria: no major impact on metabolic control. Eur J Clin Nutr. 2012;66(5):633-8.

192. Acosta PB, Wenz E, Williamson M. Nutrient intake of treated infants with phenylketonuria. Am J Clin Nutr. 1977;30(2):198-208.

193. Burlina A, Blau N. Effect of BH(4) supplementation on phenylalanine tolerance. J Inherit Metab Dis. 2009;32(1):40-5.

194. Keil S, Anjema K, van Spronsen FJ, Lambruschini N, Burlina A, BelangerQuintana A, et al. Long-term follow-up and outcome of phenylketonuria patients on sapropterin: a retrospective study. Pediatrics. 2013;131(6):e1881-8.

195. van Rijn M, Hoeksma M, Sauer PJ, Modderman P, Reijngoud DJ, van Spronsen FJ. Adult patients with well-controlled phenylketonuria tolerate incidental additional intake of phenylalanine. Ann Nutr Metab. 2011;58(2): 94-100.

196. Acosta PB, Yannicelli S. Protein intake affects phenylalanine requirements and growth of infants with phenylketonuria. Acta Paediatr Suppl. 1994;407: $66-7$

197. Kindt E, Motzfeldt K, Halvorsen S, Lie SO. Protein requirements in infants and children: a longitudinal study of children treated for phenylketonuria. Am J Clin Nutr. 1983;37(5):778-85.

198. Macdonald A, Davies P, Daly A, Hopkins V, Hall SK, Asplin D, et al. Does maternal knowledge and parent education affect blood phenylalanine control in phenylketonuria? J Hum Nutr Diet. 2008:21(4):351-8.

199. Hennermann JB, Roloff S, Gellermann J, Vollmer I, Windt E, Vetter B, et al. Chronic kidney disease in adolescent and adult patients with phenylketonuria. J Inherit Metab Dis. 2013;36(5):747-56.

200. MacDonald A. Diet and compliance in phenylketonuria. Eur J Pediatr. 2000; 159(Suppl 2):S136-41.

201. Yi SH, Singh RH. Protein substitute for children and adults with phenylketonuria. Cochrane Database Syst Rev. 2015;2:CD004731.

202. FAO/WHO/UNU. Protein and amino acid requirements in human nutrition World Health Organ Tech Rep Ser. 2007:935:1-265. back cover

203. FAO. Dietary protein quality evaluation in human nutrition. Report of an FAO Expert Consultation, 2013. From a meeting on 31-2 April, 2011. Auckland, New Zealand. FOA Food and Nutrition Paper 92. 2013.

204. van Spronsen FJ, Hoeksma M, Reijngoud DJ. Brain dysfunction in phenylketonuria: is phenylalanine toxicity the only possible cause? J Inherit Metab Dis. 2009;32(1):46-51.

205. Acosta PB, Trahms C, Wellman NS, Williamson M. Phenylalanine intakes of 1 to 6-year-old children with phenylketonuria undergoing therapy. Am J Clin Nutr. 1983;38(5):694-700.

206. Acosta PB, Yannicelli S, Marriage B, Mantia C, Gaffield B, Porterfield M, et al. Nutrient intake and growth of infants with phenylketonuria undergoing therapy. J Pediatr Gastroenterol Nutr. 1998;27(3):287-91.

207. Acosta PB, Yannicelli S, Singh R, Mofidi S, Steiner R, DeVincentis E, et al. Nutrient intakes and physical growth of children with phenylketonuria undergoing nutrition therapy. J Am Diet Assoc. 2003;103(9):1167-73.

208. Aldamiz-Echevarria L, Bueno MA, Couce ML, Lage S, Dalmau J, Vitoria I, et al. Tetrahydrobiopterin therapy vs phenylalanine-restricted diet: impact on growth in PKU. Mol Genet Metab. 2013;109(4):331-8.

209. Allen JR, Baur LA, Waters DL, Humphries IR, Allen BJ, Roberts DC, et al. Body protein in prepubertal children with phenylketonuria. Eur J Clin Nutr. 1996; 50(3):178-86.

210. Arnold GL, Vladutiu CJ, Kirby RS, Blakely EM, Deluca JM. Protein insufficiency and linear growth restriction in phenylketonuria. J Pediatr. 2002;141(2):243-6.

211. Belanger-Quintana A, Martinez-Pardo M. Physical development in patients with phenylketonuria on dietary treatment: a retrospective study. Mol Genet Metab. 2011;104(4):480-4. 
212. Dhondt JL, Largilliere C, Moreno L, Farriaux JP. Physical growth in patients with phenylketonuria. J Inherit Metab Dis. 1995;18(2):135-7.

213. Dobbelaere D, Michaud L, Debrabander A, Vanderbecken S, Gottrand F, Turck D, et al. Evaluation of nutritional status and pathophysiology of growth retardation in patients with phenylketonuria. J Inherit Metab Dis. 2003:26(1):1-11.

214. Hoeksma M, Van Rijn M, Verkerk PH, Bosch AM, Mulder MF, de Klerk JB, et al. The intake of total protein, natural protein and protein substitute and growth of height and head circumference in Dutch infants with phenylketonuria. J Inherit Metab Dis. 2005;28(6):845-54.

215. Huemer M, Huemer C, Moslinger D, Huter D, Stockler-Ipsiroglu S. Growth and body composition in children with classical phenylketonuria: results in 34 patients and review of the literature. J Inherit Metab Dis. 2007;30(5):6949.

216. Rocha JC, van Spronsen FJ, Almeida MF, Ramos E, Guimaraes JT, Borges N. Early dietary treated patients with phenylketonuria can achieve normal growth and body composition. Mol Genet Metab. 2013;110 Suppl:S40-3.

217. Schaefer F, Burgard P, Batzler U, Rupp A, Schmidt H, Gilli G, et al. Growth and skeletal maturation in children with phenylketonuria. Acta Paediatr. 1994;83(5):534-41.

218. van Spronsen FJ, Verkerk PH, van Houten M, Smit GP, van der Meer SB, Bakker HD, et al. Does impaired growth of PKU patients correlate with the strictness of dietary treatment? National Dutch PKU steering committee. Acta Paediatr. 1997;86(8):816-8.

219. Verkerk PH, van Spronsen FJ, Smit GP, Sengers RC. Impaired prenatal and postnatal growth in Dutch patients with phenylketonuria. The national PKU steering committee. Arch Dis Child. 1994;71(2):114-8

220. Weglage J, Bramswig JH, Koch HG, Karassalidou S, Ullrich K. Growth in patients with phenylketonuria. Eur J Pediatr. 1994;153(7):537-8.

221. FAO/WHO/UNU. Energy and protein requirements, Report of a Joint FAO/ WHO/UNU Expert Consultation. Geneva: World Health Organization; 1985. Contract No.: WHO Technical Report Series 724

222. Aguiar A, Ahring K, Almeida MF, Assoun M, Belanger Quintana A, Bigot S, et al. Practices in prescribing protein substitutes for PKU in Europe: no uniformity of approach. Mol Genet Metab. 2015;115(1):17-22.

223. Acosta PB. Recommendations for protein and energy intakes by patients with phenylketonuria. Eur J Pediatr. 1996;155(Suppl 1):S121-4.

224. Wu G. Amino acids: metabolism, functions, and nutrition. Amino Acids. 2009;37(1):1-17.

225. Gropper SS, Acosta PB. Effect of simultaneous ingestion of L-amino acids and whole protein on plasma amino acid and urea nitrogen concentrations in humans. JPEN J Parenter Enteral Nutr. 1991;15(1):48-53.

226. Gropper SSS, J.L. Advanced nutrition and human metabolism,Wadsworth Cengage Learning. 2013. California, USA, pp California USA: Wadsworth, Cengage Learning; 2013.

227. Jones BJ, Lees R, Andrews J, Frost P, Silk DB. Comparison of an elemental and polymeric enteral diet in patients with normal gastrointestinal function. Gut. 1983;24(1):78-84.

228. Daenzer M, Petzke KJ, Bequette BJ, Metges CC. Whole-body nitrogen and splanchnic amino acid metabolism differ in rats fed mixed diets containing casein or its corresponding amino acid mixture. J Nutr. 2001;131(7):1965-72.

229. Monch E, Herrmann ME, Brosicke H, Schoffer A, Keller M. Utilisation of amino acid mixtures in adolescents with phenylketonuria. Eur J Pediatr. 1996;155(Suppl 1):S115-20.

230. Metges CC, El-Khoury AE, Selvaraj AB, Tsay RH, Atkinson A, Regan MM, et al. Kinetics of $L-[1-(13) C]$ leucine when ingested with free amino acids, unlabeled or intrinsically labeled casein. Am J Physiol Endocrinol Metab. 2000;278(6):E1000-9.

231. Acosta PBMK. Nutritional Management of Patients with Inherited Disorders of Aromatic Amino Acid Metabolism. In: Acosta PB, editor. Nutritional Management of Patients with Inherited Metabolic Disorders. Boston: Jones and Bartlett; 2010. p. 56

232. Duran GP, Rohr FJ, Slonim A, Guttler F, Levy HL. Necessity of complete intake of phenylalanine-free amino acid mixture for metabolic control of phenylketonuria. J Am Diet Assoc. 1999;99(12):1559-63.

233. MacDonald A, Chakrapani A, Hendriksz C, Daly A, Davies P, Asplin D, et al. Protein substitute dosage in PKU: how much do young patients need? Arch Dis Child. 2006;91(7):588-93.

234. MacDonald A, Rylance G, Davies P, Asplin D, Hall SK, Booth IW. Administration of protein substitute and quality of control in phenylketonuria: a randomized study. J Inherit Metab Dis. 2003;26(4):319-26.
235. Schindeler S, Ghosh-Jerath S, Thompson S, Rocca A, Joy P, Kemp A, et al. The effects of large neutral amino acid supplements in PKU: an MRS and neuropsychological study. Mol Genet Metab. 2007;91(1):48-54.

236. Hidalgo IJ, Borchardt RT. Transport of a large neutral amino acid (phenylalanine) in a human intestinal epithelial cell line: Caco-2. Biochim Biophys Acta. 1990;1028(1):25-30.

237. Matalon R, Michals-Matalon K, Bhatia G, Burlina AB, Burlina AP, Braga C, et al Double blind placebo control trial of large neutral amino acids in treatment of PKU: effect on blood phenylalanine. J Inherit Metab Dis. 2007;30(2):153-8.

238. Matalon R, Michals-Matalon K, Bhatia G, Grechanina E, Novikov P, McDonald $J D$, et al. Large neutral amino acids in the treatment of phenylketonuria (PKU). J Inherit Metab Dis. 2006;29(6):732-8.

239. Pardridge WM. Blood-brain barrier carrier-mediated transport and brain metabolism of amino acids. Neurochem Res. 1998;23(5):635-44.

240. Pietz J, Kreis R, Rupp A, Mayatepek E, Rating D, Boesch C, et al. Large neutral amino acids block phenylalanine transport into brain tissue in patients with phenylketonuria. J Clin Invest. 1999;103(8):1169-78.

241. Pratt OE. A new approach to the treatment of phenylketonuria. J Ment Defic Res. 1980;24(3):203-17.

242. van Vliet $D$, Bruinenberg VM, Mazzola PN, van Faassen MH, de Blaauw $P$, Kema IP, et al. Large neutral amino acid supplementation exerts its effect through three synergistic mechanisms: proof of principle in Phenylketonuria mice. PLoS One. 2015;10(12):e0143833.

243. MacDonald ARG, Asplin D, Harris G, Booth IW. Abnormal feeding behaviours in phenylketonuria. J Hum Nut Diet. 1997;10:163-70.

244. Kilpatrick NM, Awang H, Wilcken B, Christodoulou J. The implication of phenylketonuria on oral health. Pediatr Dent. 1999;21(7):433-7.

245. Gokmen-Ozel H, Ferguson C, Evans S, Daly A, MacDonald A. Does a lower carbohydrate protein substitute impact on blood phenylalanine control, growth and appetite in children with PKU? Mol Genet Metab. 2011;104 Suppl:S64-7.

246. Gokmen-Ozel H, MacDonald A, Daly A, Hall K, Ryder L, Chakrapani A. Longterm efficacy of 'ready-to-drink' protein substitute in phenylketonuria. J Hum Nutr Diet. 2009;22(5):422-7.

247. levers-Landis CE, Hoff AL, Brez C, Cancilliere MK, McConnell J, Kerr D. Situational analysis of dietary challenges of the treatment regimen for children and adolescents with phenylketonuria and their primary caregivers. J Dev Behav Pediatr. 2005;26(3):186-93.

248. Prince AP, McMurray MP, Buist NR. Treatment products and approaches for phenylketonuria: improved palatability and flexibility demonstrate safety, efficacy and acceptance in US clinical trials. J Inherit Metab Dis. 1997;20(4): 486-98.

249. MacDonald A, Lilburn M, Cochrane B, Davies P, Daly A, Asplin D, et al. A new, low-volume protein substitute for teenagers and adults with phenylketonuria. J Inherit Metab Dis. 2004;27(2):127-35.

250. MacDonald A, Lilburn M, Davies P, Evans S, Daly A, Hall SK, et al. 'Ready to drink' protein substitute is easier is for people with phenylketonuria. J Inherit Metab Dis. 2006;29(4):526-31.

251. Evans S, Daly A, Chahal S, MacDonald J, MacDonald A. Food acceptance and neophobia in children with phenylketonuria: a prospective controlled study. J Hum Nutr Diet. 2016;29(4):427-33.

252. Ney DM, Gleason ST, van Calcar SC, MacLeod EL, Nelson KL, Etzel MR, et al. Nutritional management of PKU with glycomacropeptide from cheese whey. J Inherit Metab Dis. 2009;32(1):32-9.

253. van Calcar SC, MacLeod EL, Gleason ST, Etzel MR, Clayton MK, Wolff JA, et al. Improved nutritional management of phenylketonuria by using a diet containing glycomacropeptide compared with amino acids. Am J Clin Nutr. 2009;89(4):1068-77.

254. MacLeod EL, Clayton MK, van Calcar SC, Ney DM. Breakfast with glycomacropeptide compared with amino acids suppresses plasma ghrelin levels in individuals with phenylketonuria. Mol Genet Metab. 2010;100(4):303-8.

255. Allen JR, McCauley JC, Waters DL, O'Connor J, Roberts DC, Gaskin KJ. Resting energy expenditure in children with phenylketonuria. Am J Clin Nutr. 1995;62(4):797-801

256. MacDonald A. Diet and PKU [PHD]: University of Birmingham; 1999.

257. Rocha JC, van Spronsen FJ, Almeida MF, Soares G, Quelhas D, Ramos E, et al. Dietary treatment in phenylketonuria does not lead to increased risk of obesity or metabolic syndrome. Mol Genet Metab. 2012;107(4):659-63.

258. Burrage LC, McConnell J, Haesler R, O'Riordan MA, Sutton VR, Kerr DS, et al. High prevalence of overweight and obesity in females with phenylketonuria. Mol Genet Metab. 2012;107(1-2):43-8. 
259. McBurnie MA, Kronmal RA, Schuett VE, Koch R, Azeng CG. Physical growth of children treated for phenylketonuria. Ann Hum Biol. 1991;18(4):357-68.

260. Garza C, Scrimshaw NS, Young VR. Human protein requirements: the effect of variations in energy intake within the maintenance range. Am J Clin Nutr. 1976;29(3):280-7.

261. Illsinger S, Lucke T, Meyer U, Vaske B, Das AM. Branched chain amino acids as a parameter for catabolism in treated phenylketonuria. Amino Acids. 2005;28(1):45-50.

262. Todd KS, Butterfield GE, Calloway DH. Nitrogen balance in men with adequate and deficient energy intake at three levels of work. J Nutr. 1984; 114(11):2107-18

263. lyengar A, Narasinga Rao BS. Effect of varying energy and protein intake on nitrogen balance in adults engaged in heavy manual labour. Br J Nutr. 1979; 41(1):19-25

264. Iyengar AK, Rao BS, Reddy V. Nitrogen balance in Indian preschool children receiving the safe level of protein at varying levels of energy. $\mathrm{Br} J$ Nutr. 1981;46(2):295-300.

265. Jackson AA, Golden MH, Byfield R, Jahoor F, Royes J, Soutter L. Whole-body protein turnover and nitrogen balance in young children at intakes of protein and energy in the region of maintenance. Hum Nutr Clin Nutr. 1983;37(6):433-46.

266. Galli C, Agostoni C, Mosconi C, Riva E, Salari PC, Giovannini M. Reduced plasma C-20 and C-22 polyunsaturated fatty acids in children with phenylketonuria during dietary intervention. J Pediatr. 1991;119(4):562-7.

267. Rose HJ, White F, Macdonald A, Rutherford PJ, Favre E. Fat intakes of children with PKU on low phenylalanine diets. J Hum Nutr Diet. 2005;18(5): 395-400.

268. Koletzko B, Beblo S, Demmelmair H, Hanebutt FL. Omega-3 LC-PUFA supply and neurological outcomes in children with phenylketonuria (PKU). J Pediatr Gastroenterol Nutr. 2009;48(Suppl 1):S2-7.

269. Giovannini M, Agostoni C, Biasucci G, Rottoli A, Luotti D, Trojan S, et al. Fatty acid metabolism in phenylketonuria. Eur J Pediatr. 1996;155(Suppl 1):S132-5.

270. Poge AP, Baumann K, Muller E, Leichsenring M, Schmidt H, Bremer HJ. Long-chain polyunsaturated fatty acids in plasma and erythrocyte membrane lipids of children with phenylketonuria after controlled linoleic acid intake. J Inherit Metab Dis. 1998;21(4):373-81.

271. Sanjurjo P, Perteagudo L, Rodriguez Soriano J, Vilaseca A, Campistol J. Polyunsaturated fatty acid status in patients with phenylketonuria. J Inherit Metab Dis. 1994;17(6):704-9.

272. Agostoni C, Massetto N, Biasucci G, Rottoli A, Bonvissuto M, Bruzzese MG, et al. Effects of long-chain polyunsaturated fatty acid supplementation on fatty acid status and visual function in treated children with hyperphenylalaninemia. J Pediatr. 2000;137(4):504-9.

273. Beblo S, Reinhardt H, Demmelmair H, Muntau AC, Koletzko B. Effect of fish oil supplementation on fatty acid status, coordination, and fine motor skills in children with phenylketonuria. J Pediatr. 2007;150(5):479-84.

274. Cochrane B, Schwahn B, Galloway P, Robinson P, Gerasimidis K. A questionnaire survey on the usage of low protein staple foods by people with phenylketonuria in Scotland. J Hum Nutr Diet. 2014;27(6):533-41.

275. Weetch E, Macdonald A. The determination of phenylalanine content of foods suitable for phenylketonuria. J Hum Nutr Diet. 2006;19(3):229-36.

276. MacDonald A, Rylance G, Davies P, Asplin D, Hall SK, Booth IW. Free use of fruits and vegetables in phenylketonuria. J Inherit Metab Dis. 2003;26(4):327-38.

277. Rohde C, Mutze U, Schulz S, Thiele AG, Ceglarek U, Thiery J, et al. Unrestricted fruits and vegetables in the PKU diet: a 1-year follow-up. Eur J Clin Nutr. 2014;68(3):401-3

278. Zimmermann M, Jacobs $P$, Fingerhut $R$, Torresani $T$, Thony B, Blau $N$, et al. Positive effect of a simplified diet on blood phenylalanine control in different phenylketonuria variants, characterized by newborn $\mathrm{BH} 4$ loading test and PAH analysis. Mol Genet Metab. 2012;106(3):264-8.

279. MacDonald A, Depondt E, Evans S, Daly A, Hendriksz C, Chakrapani AA, et al. Breast feeding in IMD. J Inherit Metab Dis. 2006;29(2-3):299-303.

280. Agostoni C, Verduci E, Massetto N, Radaelli G, Riva E, Giovannini M. Plasma long-chain polyunsaturated fatty acids and neurodevelopment through the first 12 months of life in phenylketonuria. Dev Med Child Neurol. 2003;45(4): 257-61.

281. Banta-Wright SA, Shelton KC, Lowe ND, Knafl KA, Houck GM. Breast-feeding success among infants with phenylketonuria. J Pediatr Nurs. 2012;27(4):319-27.

282. Francis DEM SI. Breast-feeding regime for the treatment of infants with phenylketonuria. In: C. B, editor. Applied Nutrition. London: John Libbey; 1981. p. 82-83.
283. McCabe L, Ernest AE, Neifert MR, Yannicelli S, Nord AM, Garry PJ, et al. The management of breast feeding among infants with phenylketonuria. J Inherit Metab Dis. 1989;12(4):467-74.

284. Motzfeldt K, Lilje R, Nylander G. Breastfeeding in phenylketonuria. Acta Paediatr Suppl. 1999;88(432):25-7.

285. van Rijn M, Bekhof J, Dijkstra T, Smit PG, Moddermam P, van Spronsen FJ. A different approach to breast-feeding of the infant with phenylketonuria. Eur J Pediatr. 2003;162(5):323-6.

286. Humphries P, Pretorius $E$, Naude $H$. Direct and indirect cellular effects of aspartame on the brain. Eur J Clin Nutr. 2008;62(4):451-62.

287. Trefz F, de Sonneville L, Matthis P, Benninger C, Lanz-Englert B, Bickel H. Neuropsychological and biochemical investigations in heterozygotes for phenylketonuria during ingestion of high dose aspartame (a sweetener containing phenylalanine). Hum Genet. 1994;93(4):369-74.

288. Caballero B, Mahon BE, Rohr FJ, Levy HL, Wurtman RJ. Plasma amino acid levels after single-dose aspartame consumption in phenylketonuria, mild hyperphenylalaninemia, and heterozygous state for phenylketonuria. J Pediatr. 1986;109(4):668-71.

289. Koch R, Shaw KN, Williamson M, Haber M. Use of aspartame in phenylketonuric heteroxygous adults. J Toxicol Environ Health. 1976;2(2):453-7.

290. Mackey SA, Berlin CM Jr. Effect of dietary aspartame on plasma concentrations of phenylalanine and tyrosine in normal and homozygous phenylketonuric patients. Clin Pediatr. 1992;31(7):394-9.

291. Stegink LD, Filer LJ Jr, Baker GL, Bell EF, Ziegler EE, Brummel MC, et al. Repeated ingestion of aspartame-sweetened beverage: effect on plasma amino acid concentrations in individuals heterozygous for phenylketonuria. Metab Clin Exp. 1989:38(1):78-84

292. Stegink LD, Filer LJ Jr, Bell EF, Ziegler EE, Tephly TR, Krause WL. Repeated ingestion of aspartame-sweetened beverages: further observations in individuals heterozygous for phenylketonuria. Metab Clin Exp. 1990;39(10): 1076-81.

293. Stegink LD, Koch R, Blaskovics ME, Filer LJ Jr, Baker GL, McDonnell JE. Plasma phenylalanine levels in phenylketonuric heterozygous and normal adults administered aspartame at $34 \mathrm{mg} / \mathrm{kg}$ body weight. Toxicology. 1981; 20(1):81-90.

294. Stegink LD, Wolf-Novak LC, Filer LJ Jr, Bell EF, Ziegler EE, Krause WL, et al. Aspartame-sweetened beverage: effect on plasma amino acid concentrations in normal adults and adults heterozygous for phenylketonuria. J Nutr. 1987;117(11):1989-95.

295. Wolf-Novak LC, Stegink LD, Brummel MC, Persoon TJ, Filer LJ Jr, Bell EF, et al. Aspartame ingestion with and without carbohydrate in phenylketonuric and normal subjects: effect on plasma concentrations of amino acids, glucose, and insulin. Metab Clin Exp. 1990;39(4):391-6.

296. Chattopadhyay S, Raychaudhuri U, Chakraborty R. Artificial sweeteners - a review. J Food Sci Technol. 2014;51(4):611-21.

297. van Spronsen FJ, van Rijn M, Bekhof J, Koch R, Smit PG. Phenylketonuria: tyrosine supplementation in phenylalanine-restricted diets. Am J Clin Nutr. 2001;73(2):153-7.

298. Bross R, Ball RO, Clarke JT, Pencharz PB. Tyrosine requirements in children with classical PKU determined by indicator amino acid oxidation. Am J Physiol Endocrinol Metab. 2000;278(2):E195-201.

299. Sharman R, Sullivan KA, Young RM, McGill JJ. Tyrosine monitoring in children with early and continuously treated phenylketonuria: results of an international practice survey. J Inherit Metab Dis. 2010;33(Suppl 3):S417-20.

300. Mazzocco MM, Yannicelli S, Nord AM, van Doorninck W, Davidson-Mundt AJ, Greene CL. Cognition and tyrosine supplementation among schoolaged children with phenylketonuria. Am J Dis Child. 1992;146(11):1261-4.

301. Pietz J, Landwehr R, Kutscha A, Schmidt H, de Sonneville L, Trefz FK. Effect of high-dose tyrosine supplementation on brain function in adults with phenylketonuria. J Pediatr. 1995;127(6):936-43.

302. Smith ML, Hanley WB, Clarke JT, Klim P, Schoonheyt W, Austin V, et al. Randomised controlled trial of tyrosine supplementation on neuropsychological performance in phenylketonuria. Arch Dis Child. 1998; 78(2):116-21.

303. Webster D, Wildgoose J. Tyrosine supplementation for phenylketonuria. Cochrane Database Syst Rev. 2013;6:CD001507.

304. Moats RA, Moseley KD, Koch R, Nelson M Jr. Brain phenylalanine concentrations in phenylketonuria: research and treatment of adults. Pediatrics. 2003;112(6 Pt 2):1575-9.

305. Christensen HN. Metabolism of amino acids and proteins. Annu Rev Biochem. 1953;22:233-60. 
306. Gardiner K, Barbul A. Intestinal amino acid absorption during sepsis. JPEN Parenter Enteral Nutr. 1993;17(3):277-83.

307. Smith JSSW. Nutritional aspects of trauma, post surgical care. In: F. B, editor. Nutritional aspects of clinical management of chronic disorders and diseases. Florida: CRC press; 2003. p. 227-63.

308. Keusch GT, Farthing MJ. Nutrition and infection. Annu Rev Nutr. 1986:6:131-54

309. Dubois E. The basal metabolism in fever. J Am Med Assoc. 1921;77:352-5.

310. Tulikoura I, Huikuri K. Changes in nitrogen metabolism in catabolic patients given three different parenteral nutrition regimens. Acta Chir Scand. 1981; 147(7):519-24

311. Trefz FK, Scheible D, Frauendienst-Egger G. Long-term follow-up of patients with phenylketonuria receiving tetrahydrobiopterin treatment. J Inherit Metab Dis. 2010;33(Suppl 3):S163-9.

312. Ballhausen D, Egli D, Bickle-Graz M, Bianchi N, Bonafe L. Born at 27 weeks of gestation with classical PKU: challenges of dietetic management in a very preterm infant. Pediatric Rep. 2011;3(4):e26.

313. Cole DE, Landry DA. Parenteral nutrition in a premature infant with phenylketonuria. JPEN J Parenter Enteral Nutr. 1984;8(1):42-4.

314. Lin HJ, Kwong AM, Carter JM, Ferreira BF, Austin MF, Devarajan K, et al. Extremely high phenylalanine levels in a newborn on parenteral nutrition: phenylketonuria in the neonatal intensive care unit. J Perinatol: official journal of the California Perinatal Association. 2011;31(7):507-10.

315. Salvarinova-Zivkovic R, Hartnett C, Sinclair G, Dix D, Horvath G, Lillquist $Y$, et al. The use of parenteral nutrition for the management of PKU patient undergoing chemotherapy for lymphoma: a case report. Mol Genet Metab. 2012;105(4):571-4

316. Kaluzny L, Szczepanik M, Siwinska-Mrozek Z, Borkowska-Klos M, Cichy W, Walkowiak J. Parenteral nutrition in patients with inborn errors of metabolism - a therapeutic problem. Eur Rev Med Pharmacol Sci. 2014; 18(11):1579-82.

317. Antisdel JE, Chrisler JC. Comparison of eating attitudes and behaviors among adolescent and young women with type 1 diabetes mellitus and phenylketonuria. J Dev Behav Pediatr. 2000;21(2):81-6.

318. Lenke RR, Levy HL. Maternal phenylketonuria and hyperphenylalaninemia. An international survey of the outcome of untreated and treated pregnancies. N Engl J Med. 1980;303(21):1202-8.

319. Koch R, Hanley W, Levy H, Matalon K, Matalon R, Rouse B, et al. The maternal Phenylketonuria international study: 1984-2002. Pediatrics. 2003; 112(6 Pt 2):1523-9.

320. Levy HL, Guldberg P, Guttler F, Hanley WB, Matalon R, Rouse BM, et al. Congenital heart disease in maternal phenylketonuria: report from the maternal PKU collaborative study. Pediatr Res. 2001;49(5):636-42.

321. Platt LD, Koch R, Hanley WB, Levy HL, Matalon R, Rouse B, et al. The international study of pregnancy outcome in women with maternal phenylketonuria: report of a 12-year study. Am J Obstet Gynecol. 2000; 182(2):326-33.

322. Rouse B, Matalon R, Koch R, Azen C, Levy H, Hanley W, et al. Maternal phenylketonuria syndrome: congenital heart defects, microcephaly, and developmental outcomes. J Pediatr. 2000;136(1):57-61.

323. Rouse B, Azen C, Koch R, Matalon R, Hanley W, de la Cruz F, et al. Maternal Phenylketonuria collaborative study (MPKUCS) offspring: facial anomalies, malformations, and early neurological sequelae. Am J Med Genet. 1997; 69(1):89-95.

324. Levy HL, Waisbren SE, Lobbregt D, Allred E, Schuler A, Trefz FK, et al. Maternal mild hyperphenylalaninaemia: an international survey of offspring outcome. Lancet. 1994;344(8937):1589-94.

325. Levy HL, Waisbren SE, Guttler F, Hanley WB, Matalon R, Rouse B, et al. Pregnancy experiences in the woman with mild hyperphenylalaninemia. Pediatrics. 2003;112(6 Pt 2):1548-52

326. Waisbren SE, Azen C. Cognitive and behavioral development in maternal phenylketonuria offspring. Pediatrics. 2003;112(6 Pt 2):1544-7.

327. Lee PJ, Ridout D, Walter JH, Cockburn F. Maternal phenylketonuria: report from the United Kingdom registry 1978-97. Arch Dis Child. 2005;90(2):143-6.

328. Prick BW, Hop WC, Duvekot JJ. Maternal phenylketonuria and hyperphenylalaninemia in pregnancy: pregnancy complications and neonatal sequelae in untreated and treated pregnancies. Am J Clin Nutr. 2012;95(2):374-82.

329. Rouse B, Azen C. Effect of high maternal blood phenylalanine on offspring congenital anomalies and developmental outcome at ages 4 and 6 years: the importance of strict dietary control preconception and throughout pregnancy. J Pediatr. 2004;144(2):235-9.
330. Maillot F, Lilburn M, Baudin J, Morley DW, Lee PJ. Factors influencing outcomes in the offspring of mothers with phenylketonuria during pregnancy: the importance of variation in maternal blood phenylalanine. Am J Clin Nutr. 2008:88(3):700-5.

331. Teissier R, Nowak E, Assoun M, Mention K, Cano A, Fouilhoux A, et al. Maternal phenylketonuria: low phenylalaninemia might increase the risk of intra uterine growth retardation. J Inherit Metab Dis. 2012;35(6):993-9.

332. Cooperative GH. Prenatal care screening and testing guideline. https://www. ghc.org/all-sites/guidelines/prenatal.pdf: Group Health Cooperative 2013. p. 16.

333. Maillot F, Cook P, Lilburn M, Lee PJ. A practical approach to maternal phenylketonuria management. J Inherit Metab Dis. 2007;30(2):198-201.

334. Chetty SP, Shaffer BL, Norton ME. Management of pregnancy in women with genetic disorders: part 2: inborn errors of metabolism, cystic fibrosis, neurofibromatosis type 1, and turner syndrome in pregnancy. Obstet Gynecol Surv. 2011;66(12):765-76.

335. Rohr F, Munier A, Sullivan D, Bailey I, Gennaccaro M, Levy H, et al. The resource mothers study of maternal Phenylketonuria: preliminary findings. J Inherit Metab Dis. 2004;27(2):145-55.

336. Hall JG. When is careless conception a form of child abuse? Lessons from maternal phenylketonuria. J Pediatr. 2000;136(1):12-3.

337. Singh S, Sedgh G, Hussain R. Unintended pregnancy: worldwide levels, trends, and outcomes. Stud Fam Plan. 2010;41(4):241-50.

338. Koch R, Trefz F, Waisbren S. Psychosocial issues and outcomes in maternal PKU. Mol Genet Metab. 2010;99(Suppl 1):S68-74.

339. Levy HL, Ghavami M. Maternal phenylketonuria: a metabolic teratogen. Teratology. 1996;53(3):176-84.

340. Waisbren SE, Shiloh S, St James P, Levy HL. Psychosocial factors in maternal phenylketonuria: prevention of unplanned pregnancies. Am J Public Health. 1991;81(3):299-304

341. Charron-Prochownik D, Ferons-Hannan M, Sereika S, Becker D. Randomized efficacy trial of early preconception counseling for diabetic teens (READYgirls). Diabetes Care. 2008;31(7):1327-30.

342. Fischl AF, Herman WH, Sereika SM, Hannan M, Becker D, Mansfield MJ, et al. Impact of a preconception counseling program for teens with type 1 diabetes (READY-girls) on patient-provider interaction, resource utilization, and cost. Diabetes Care. 2010;33(4):701-5.

343. Kohlschutter B, Ellerbrok M, Merkel M, Tchirikov M, Zschocke J, Santer R, et al. Phenylalanine tolerance in three phenylketonuric women pregnant with fetuses of different genetic PKU status. J Inherit Metab Dis. 2009:32(Suppl 1): $\mathrm{S} 1-4$

344. Trefz F, Maternal PKU. PKU and BH4-Advances in Phenylketonuria and Tetrahydrobiopterin SPS Verlagsgesellschaft. In: Blau N, editor. ; 2006. p. 261-176.

345. Matalon KM, Acosta PB, Azen C. Role of nutrition in pregnancy with phenylketonuria and birth defects. Pediatrics. 2003;112(6 Pt 2):1534-6.

346. Siega-Riz AM, Viswanathan M, Moos MK, Deierlein A, Mumford S, Knaack J, et al. A systematic review of outcomes of maternal weight gain according to the Institute of Medicine recommendations: birthweight, fetal growth, and postpartum weight retention. Am J Obstet Gynecol. 2009;201((4):339): e1-14.

347. Clark BJ, Cockburn F. Management of inborn errors of metabolism during pregnancy. Acta Paediatr Scand Suppl. 1991;373:43-52.

348. Michals K, Acosta PB, Austin V, Castiglioni L, Rohr F, Wenz E, et al. Nutrition and reproductive outcome in maternal phenylketonuria. Eur J Pediatr. 1996; 155(Suppl 1):S165-8.

349. Feillet F, Abadie V, Berthelot J, Maurin N, Ogier H, Vidailhet M, et al. Maternal phenylketonuria: the French survey. Eur J Pediatr. 2004;163(9):540-6.

350. Acosta PB, Matalon K, Castiglioni L, Rohr FJ, Wenz E, Austin V, et al. Intake of major nutrients by women in the maternal Phenylketonuria (MPKU) study and effects on plasma phenylalanine concentrations. Am J Clin Nutr. 2001; 73(4):792-6.

351. Thompson GN, Francis DE, Kirby DM, Compton R. Pregnancy in phenylketonuria: dietary treatment aimed at normalising maternal plasma phenylalanine concentration. Arch Dis Child. 1991;66(11):1346-9.

352. Butte NF, King JC. Energy requirements during pregnancy and lactation. Public Health Nutr. 2005;8(7A):1010-27.

353. Nutrition TSACo. Dietary reference values for energy. SACN reports and position statements [internet]. 2011.

354. WHO. Maternal anthropometry and pregnancy outcomes. A WHO collaborative study. Bull World Health Organ. 1995;73 Suppl:1-98. 
355. Maritz CMC H, Ellerton C. Dietary management of Maternal Phenylketonuria: A practical guide; 2010. p. 1-27

356. Andreasen KR, Andersen ML, Schantz AL. Obesity and pregnancy. Acta Obstet Gynecol Scand. 2004;83(11):1022-9.

357. Morin KH. Perinatal outcomes of obese women: a review of the literature. J Obstet Gynecol Neonatal Nurs. 1998;27(4):431-40.

358. Coutts J. The dietary management of phenylketonuria. Proc Nutr Soc. 1979; 38(3):315-20

359. Michals-Matalon KMR, Acosta P, Azen C. Congenital heart disease in maternal phenylketonuria: effects of blood phenylalanine and nutrient intake. MRDD Res Rev. 1999;5:121-4

360. Yano S, Moseley K, Bottiglieri T, Arning E, Azen C. Maternal Phenylketonuria international collaborative study revisited: evaluation of maternal nutritional risk factors besides phenylalanine for fetal congenital heart defects. J Inherit Metab Dis. 2014;37(1):39-42.

361. Singh RH, Rohr F, Frazier D, Cunningham A, Mofidi S, Ogata B, et al. Recommendations for the nutrition management of phenylalanine hydroxylase deficiency. Genet Med. 2014;16(2):121-31.

362. Acosta PB, Blaskovics M, Cloud H, Lis E, Stroud H, Wenz E. Nutrition in pregnancy of women with hyperphenylalaninemia. J Am Diet Assoc. 1982; 80(5):443-50.

363. Brenton DP, Lilburn M. Maternal phenylketonuria. A study from the United Kingdom. Eur J Pediatr. 1996;155(Suppl 1):S177-80.

364. Davidson DC. Maternal phenylketonuria. Postgrad Med J. 1989;65(Suppl 2): S10-20.

365. Koch R, Friedman EG, Wenz E, Jew K, Crowley C, Donnell G. Maternal phenylketonuria. J Inherit Metab Dis. 1986;9(Suppl 2):159-68.

366. Komrower GM, Sardharwalla IB, Coutts JM, Ingham D. Management of maternal phenylketonuria: an emerging clinical problem. Br Med J. 1979; 1(6175):1383-7.

367. Lenke RR, Koch R, Fishler K, Platt LD. Tyrosine supplementation during pregnancy in a woman with classical phenylketonuria. A case report. J Reprod Med. 1983;28(6):411-4.

368. Robertson LVM, A.; Ripley, S.; Adams, S.; Chan, H.; Ellerton, C.; Maritz, C. Mestravick, N.; Micciche, A.; Terry, A.; Weetch, E.; Wildgoose, J., editor Maternal Phenylketonuria (PKU) Practical Management in UK Metabolic Centers. SSIEM; 2010; Istanbul: Springer JIMD.

369. Rohr FJ, Doherty LB, Waisbren SE, Bailey IV, Ampola MG, Benacerraf B, et al. New England maternal PKU project: prospective study of untreated and treated pregnancies and their outcomes. J Pediatr. 1987;110(3):391-8.

370. Rohr FJ, Lobbregt D, Levy HL. Tyrosine supplementation in the treatment of maternal phenylketonuria. Am J Clin Nutr. 1998;67(3):473-6.

371. Sheil ODN, Saul IP, Naughten ER. Maternal phenylketonuria with increased tyrosine supplements. J Inherit Metab Dis. 1986;9(Suppl 2):3.

372. Bessman SP. Genetic failure of fetal amino acid "justification": a common basis for many forms of metabolic, nutritional, and "nonspecific" mental retardation. J Pediatr. 1972;81(4):834-42.

373. Levy HL, Lenke RR, Koch R. Lack of fetal effect on blood phenylalanine concentration in maternal phenylketonuria. J Pediatr. 1984;104(2):245-7.

374. Lewis SA, Lyon IC, Elliott RB. Outcome of pregnancy in the rat with mild hyperphenylalaninaemia and hypertyrosinaemia: implications for the management of human maternal PKU. J Inherit Metab Dis. 1985;8(3):113-7.

375. Maltepe C, Koren G. The management of nausea and vomiting of pregnancy and hyperemesis gravidarum-a 2013 update. J Popul Ther Clin Pharmacol. 2013:20(2):e184-92.

376. Schrag SP, Sharma R, Jaik NP, Seamon MJ, Lukaszczyk JJ, Martin ND, et al. Complications related to percutaneous endoscopic gastrostomy (PEG) tubes. A comprehensive clinical review. J Gastrointestin Liver Dis. 2007;16(4): 407-18.

377. Schwoerer JAOL, Van Calcar S, Heighway S, Bankowski H, Williams P, Rice G. Use of Gastrostomy tube to prevent maternal PKU syndrome. JIMD Rep. 2012;6:6.

378. Group MVSR. Prevention of neural tube defects: results of the Medical Research Council vitamin study. Lancet. 1991;338(8760):131-7.

379. Health. Do. Folic acid and the prevention of disease. Report of the committee on medical aspects of food and nutrition policy. In: Report on health and social subjects 50. London: The Stationery Office; 2000.

380. Wald NJ, Law M, Hoffbrand AV. Folic acid fortification in the prevention of neural tube defects. Am J Clin Nutr. 2004;80(6):1665. author reply -6

381. Foods SCo. Tolerable upper intake level of Folate. European Scientific Committee on Foods. 2000.
382. Medicine lo. Dietary reference intakes for energy, carbohydrate, fibre, fat, fatty acids, cholesterol, protein and aminoacids. Washinton, D.C.: The National Academies Press; 2005. Available from: https://www.nap.edu/read/ 10490/chapter/1

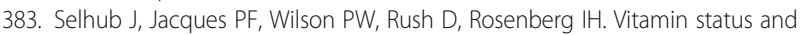
intake as primary determinants of homocysteinemia in an elderly population. JAMA. 1993;270(22):2693-8.

384. Giovannini M, Biasucci G, Agostoni C, Bellu R, Riva E. Fatty acid supplementation in a case of maternal phenylketonuria. J Inherit Metab Dis. 1994;17(5):630-1.

385. Eilander A, Hundscheid DC, Osendarp SJ, Transler C, Zock PL. Effects of n-3 long chain polyunsaturated fatty acid supplementation on visual and cognitive development throughout childhood: a review of human studies. Prostaglandins Leukot Essent Fat Acids. 2007;76(4):189-203.

386. Koletzko B, Boey CC, Campoy C, Carlson SE, Chang N, Guillermo-Tuazon MA, et al. Current information and Asian perspectives on long-chain polyunsaturated fatty acids in pregnancy, lactation, and infancy: systematic review and practice recommendations from an early nutrition academy workshop. Ann Nutr Metab. 2014;65(1):49-80.

387. Wheeler S. Assessment and interpretation of micronutrient status during pregnancy. Proc Nutr Soc. 2008;67(4):437-50.

388. Bradburn NC, Wappner RS, Lemons JA, Meyer BA, Roberts RS. Lactation and phenylketonuria. Am J Perinatol. 1985;2(2):138-41.

389. Kuvan ${ }^{\circledast}$, inventorSummary of Product Characteristics. 13/11/2013.

390. Fox-Bacon C, McCamman S, Therou L, Moore W, Kipp DE. Maternal PKU and breastfeeding: case report of identical twin mothers. Clin Pediatr. 1997;36(9): 539-42.

391. MacDonald A WF. Disorders of Amino acid Metabolism. In: V. S, editor. Clinical Paeditaric Dietetics. Wiley Blackwell; 2014.

392. Hall Moran V, Lowe N, Crossland N, Berti C, Cetin I, Hermoso M, et al. Nutritional requirements during lactation. Towards European alignment of reference values: the EURRECA network. Matern Child Nutr. 2010;6(Suppl 2): $39-54$.

393. FAO/WHO/UNU. Human energy requirements. Report of a joint FAO/WHO/ UNU expert consultation. October 17-24, 2001. Rome, Italy. Public Health Nutr. 2004:8(7A):929-1228.

394. Dewey KG. Energy and protein requirements during lactation. Annu Rev Nutr. 1997:17:19-36.

395. Centers for Disease C, Prevention. Barriers to dietary control among pregnant women with phenylketonuria-United States, 1998-2000. MMWR Morb Mortal Wkly Rep. 2002;51(6):117-20.

396. Gentile JK, Ten Hoedt AE, Bosch AM. Psychosocial aspects of PKU: hidden disabilities-a review. Mol Genet Metab. 2010;99(Suppl 1):S64-7.

397. Brown AS, Fernhoff PM, Waisbren SE, Frazier DM, Singh R, Rohr F, et al. Barriers to successful dietary control among pregnant women with phenylketonuria. Genet Med. 2002;4(2):84-9.

398. Waisbren SE, Hamilton BD, St James PJ, Shiloh S, Levy HL. Psychosocial factors in maternal phenylketonuria: women's adherence to medical recommendations. Am J Public Health. 1995;85(12):1636-41.

399. Koch R, Moseley K, Ning J, Romstad A, Guldberg P, Guttler F. Long-term beneficial effects of the phenylalanine-restricted diet in late-diagnosed individuals with phenylketonuria. Mol Genet Metab. 1999;67(2):148-55.

400. Trefz FK, Cipcic-Schmidt S, Koch R. Final intelligence in late treated patients with phenylketonuria. Eur J Pediatr. 2000;159(Suppl 2):S145-8.

401. Dan B, Christiaens F, Mewasingh LD, De Laet C, Goyens P. Late-treated phenylketonuria mimicking Angelman syndrome. Am J Med Genet. 2001; 104(4):345-6.

402. Groselj U, Tansek MZ, Smon A, Angelkova N, Anton D, Baric I, et al. Newborn screening in southeastern Europe. Mol Genet Metab. 2014;113(1-2):42-5.

403. Holtzman C, Slazyk WE, Cordero JF, Hannon WH. Descriptive epidemiology of missed cases of phenylketonuria and congenital hypothyroidism. Pediatrics. 1986;78(4):553-8.

404. Narayanan D, Barski R, Henderson MJ, Luvai A, Chandrajay D, Stainforth C, et al. Delayed diagnosis of phenylketonuria - a case report of two siblings. Ann Clin Biochem. 2014;51(Pt 3):406-8.

405. Kasim S, Moo LR, Zschocke J, Jinnah HA. Phenylketonuria presenting in adulthood as progressive spastic paraparesis with dementia. J Neurol Neurosurg Psychiatry. 2001;71(6):795-7.

406. Rosini F, Rufa A, Monti L, Tirelli L, Federico A. Adult-onset phenylketonuria revealed by acute reversible dementia, prosopagnosia and parkinsonism. J Neurol. 2014;261(12):2446-8. 
407. Murphy GH, Johnson SM, Amos A, Weetch E, Hoskin R, Fitzgerald B, et al. Adults with untreated phenylketonuria: out of sight, out of mind. $\mathrm{Br} J$ Psychiatry. 2008;193(6):501-2.

408. Gizewska M. Phenylketonuria : selected genetic aspects and sequelae of hyperhenylalaninemia. Habilitation thesis. Ann Acad Med Stetin. 2008;supl. 132.

409. Mazur A, Jarochowicz S, Oltarzewski M, Sykut-Cegielska J, Kwolek A, O'Malley G. Measurement of functional independence level and falls-risk in individuals with undiagnosed phenylketonuria. Acta Biochim Pol. 2009;56(4):613-8.

410. Jancar J. Increased life expectancy in people with untreated phenylketonuria. J Intellect Disabil Res. 1998;42(Pt 1):97-9.

411. Gizewska M, Cabalska B, Cyrytowski L, Nowacki P, Zekanowski C, Walczak M, et al. Different presentations of late-detected phenylketonuria in two brothers with the same R408W/R111X genotype in the PAH gene. J Intellect Disabil Res. 2003;47(Pt 2):146-52.

412. Grosse SD. Late-treated phenylketonuria and partial reversibility of intellectual impairment. Child Dev. 2010;81(1):200-11.

413. Holmgren G, Blomquist HK, Samuelson G. Positive effect of a late introduced modified diet in an 8-year-old pku child. Neuropadiatrie. 1980; 11(1):76-9.

414. Baumeister AA, Baumeister AA. Dietary treatment of destructive behavior associated with hyperphenylalaninemia. Clin Neuropharmacol. 1998;21(1): 18-27.

415. Bruhl HH, Arnesen JF, Bruhl MG. Effect of a low-phenylalanine diet on older Phenylketonuria patients (long range controlled study). Am J Ment Defic. 1964;69:225-35.

416. Dion EP, M.J.;, Carriere, S.; Babin, C.; Goisneau, J. Phenylalanine restricted diet treatment of the aggressive behaviours of a person with mental retardation. Br J Dev Disabil. 2001;47(1).

417. Dolan BEK, R.; Bekins, C.; Schuett, V. Diet Intervention Guidelines for Adults with Untreated PKU. http://pkunews.org/adults/guide.htm1998.

418. Fitzgerald B, Morgan J, Keene N, Rollinson R, Hodgson A, Dalrymple-Smith J. An investigation into diet treatment for adults with previously untreated phenylketonuria and severe intellectual disability. J Intellect Disabil Res. 2000:44(Pt 1):53-9.

419. Harper M, Reid AH. Use of a restricted protein diet in the treatment of behaviour disorder in a severely mentally retarded adult female phenylketonuric patient. J Ment Defic Res. 1987;31(Pt 2):209-12.

420. Hoskin RG, Sasitharan T, Howard R. The use of a low phenylalanine diet with amino acid supplement in the treatment of behavioural problems in a severely mentally retarded adult female with phenylketonuria. J Intellect Disabil Res. 1992;36(Pt 2):183-91.

421. Lee PJ, Amos A, Robertson L, Fitzgerald B, Hoskin R, Lilburn M, et al. Adults with late diagnosed PKU and severe challenging behaviour: a randomised placebo-controlled trial of a phenylalanine-restricted diet. J Neurol Neurosurg Psychiatry. 2009;80(6):631-5.

422. Marholin D 2nd, Pohl RE 3rd, Stewart RM, Touchette PE, Townsend NM, Kolodny EH. Effects of diet and behavior therapy on social and motor behavior of retarded phenylketonuric adults: an experimental analysis. Pediatr Res. 1978:12(3):179-87.

423. Merrick J, Aspler S, Schwarz G. Phenylalanine-restricted diet should be life long. A case report on long-term follow-up of an adolescent with untreated phenylketonuria. Int J Adolesc Med Health. 2003;15(2):165-8.

424. Pavone L, Meli C., Nigro F., Lisi R., Di Raimondo S., Mollica F. Late diagnosed phenylketonuria patients: clinical presentation and results of treatment. Dev Brain Dysfunct. 1993;6.

425. Williams K. Benefits of normalizing plasma phenylalanine: impact on behaviour and health. A case report. J Inherit Metab Dis. 1998:21(8):785-90.

426. Yannicelli S, Ryan A. Improvements in behaviour and physical manifestations in previously untreated adults with phenylketonuria using a phenylalanine-restricted diet: a national survey. J Inherit Metab Dis. 1995 18(2):131-4

427. Hambraeus L, Holmgren G, Samuelson G. Dietary treatment of adult patients with phenylketonuria. Nutr Metab. 1971;13(5):298-317.

428. Giffin FD, Clarke JT, d'Entremont DM. Effect of dietary phenylalanine restriction on visual attention span in mentally retarded subjects with phenylketonuria. Can J Neurol Sci. 1980;7(2):127-31.

429. Schuett VE, Brown ES, Michals K. Reinstitution of diet therapy in PKU patients from twenty-two US clinics. Am J Public Health. 1985;75(1):39-42.

430. Brown MC, Guest JF. Economic impact of feeding a phenylalanine-restricted diet to adults with previously untreated phenylketonuria. J Intellect Disabil Res. 1999:43(Pt 1):30-7.
431. Hoskin R. Dietary treatment of the untreated adult PKU. 2000. Available from: http://www.nspku.org/publications/publication/dietary-treatmentuntreated-adult-pku.

432. Kalkanoglu HS, Ahring KK, Sertkaya D, Moller LB, Romstad A, Mikkelsen I, et al. Behavioural effects of phenylalanine-free amino acid tablet supplementation in intellectually disabled adults with untreated phenylketonuria. Acta Paediatr. 2005;94(9):1218-22.

433. Vernon HJ, Koerner CB, Johnson MR, Bergner A, Hamosh A. Introduction of sapropterin dihydrochloride as standard of care in patients with phenylketonuria. Mol Genet Metab. 2010;100(3):229-33.

434. Bekhof J, van Spronsen FJ, Crone MR, van Rijn M, Oudshoorn CG, Verkerk $\mathrm{PH}$. Influence of knowledge of the disease on metabolic control in phenylketonuria. Eur J Pediatr. 2003;162(6):440-2.

435. Durham-Shearer SJ, Judd PA, Whelan K, Thomas JE. Knowledge, compliance and serum phenylalanine concentrations in adolescents and adults with phenylketonuria and the effect of a patient-focused educational resource. J Hum Nutr Diet. 2008;21(5):474-85.

436. Singh RH, Kable JA, Guerrero NV, Sullivan KM, Elsas $L J$ 2nd. Impact of a camp experience on phenylalanine levels, knowledge, attitudes, and health beliefs relevant to nutrition management of phenylketonuria in adolescent girls. J Am Diet Assoc. 2000;100(7):797-803.

437. Waisbren SE, Rokni H, Bailey I, Rohr F, Brown T, Warner-Rogers J. Social factors and the meaning of food in adherence to medical diets: results of a maternal phenylketonuria summer camp. J Inherit Metab Dis. 1997;20(1):21-7.

438. Norris SL, Engelgau MM, Narayan KM. Effectiveness of self-management training in type 2 diabetes: a systematic review of randomized controlled trials. Diabetes Care. 2001;24(3):561-87.

439. Burton BK, Leviton L. Reaching out to the lost generation of adults with early-treated phenylketonuria (PKU). Mol Genet Metab. 2010;101(2-3):146-8.

440. van Rijn M, Ahring K, Bélanger-Quintana A, Dokoupil K, Gokmen Ozel H, Lammardo AM, et al. When should social service referral be considered in phenylketonuria? Mol Genet Metab Rep. 2015;2:4.

441. Taylor A. The impact of parental substance misuse on child development. In: Research in practice; 2013.

442. Burton BK, Grange DK, Milanowski A, Vockley G, Feillet F, Crombez EA, et al. The response of patients with phenylketonuria and elevated serum phenylalanine to treatment with oral sapropterin dihydrochloride (6Rtetrahydrobiopterin): a phase II, multicentre, open-label, screening study. J Inherit Metab Dis. 2007;30(5):700-7.

443. Couce ML, Boveda MD, Valerio E, Perez-Munuzuri A, Fraga JM. Long-term pharmacological management of phenylketonuria, including patients below the age of 4 years. JIMD Rep. 2012;2:91-6.

444. Elsas $\sqcup$, Greto J, Wierenga A. The effect of blood phenylalanine concentration on Kuvan response in phenylketonuria. Mol Genet Metab. 2011:102(4):407-12.

445. Hennermann JB, Buhrer C, Blau N, Vetter B, Monch E. Long-term treatment with tetrahydrobiopterin increases phenylalanine tolerance in children with severe phenotype of phenylketonuria. Mol Genet Metab. 2005;86(Suppl 1):S86-90

446. Lambruschini N, Perez-Duenas B, Vilaseca MA, Mas A, Artuch R, Gassio R, et al. Clinical and nutritional evaluation of phenylketonuric patients on tetrahydrobiopterin monotherapy. Mol Genet Metab. 2005;86(Suppl 1):S54-60.

447. Levy HL, Milanowski A, Chakrapani A, Cleary M, Lee P, Trefz FK, et al. Efficacy of sapropterin dihydrochloride (tetrahydrobiopterin, 6R-BH4) for reduction of phenylalanine concentration in patients with phenylketonuria: a phase III randomised placebo-controlled study. Lancet. 2007;370(9586):504-10.

448. Lindegren ML, Krishnaswami S, Reimschisel T, Fonnesbeck C, Sathe NA, McPheeters ML. A systematic review of $\mathrm{BH} 4$ (Sapropterin) for the adjuvant treatment of Phenylketonuria. JIMD Rep. 2013;8:109-19.

449. Muntau AC, Roschinger W, Habich M, Demmelmair H, Hoffmann B, Sommerhoff $\mathrm{CP}$, et al. Tetrahydrobiopterin as an alternative treatment for mild phenylketonuria. N Engl J Med. 2002;347(26):2122-32.

450. Singh $\mathrm{RH}$, Quirk ME. Using change in plasma phenylalanine concentrations and ability to liberalize diet to classify responsiveness to tetrahydrobiopterin therapy in patients with phenylketonuria. Mol Genet Metab. 2011;104(4):485-91.

451. Singh RH, Quirk ME, Douglas TD, Brauchla MC. BH(4) therapy impacts the nutrition status and intake in children with phenylketonuria: 2-year followup. J Inherit Metab Dis. 2010;33(6):689-95.

452. Thiele AG, Weigel JF, Ziesch B, Rohde C, Mutze U, Ceglarek U, et al. Nutritional changes and micronutrient supply in patients with Phenylketonuria under therapy with Tetrahydrobiopterin (BH(4)). JIMD Rep. 2013:9:31-40. 
453. Zurfluh MR, Fiori L, Fiege B, Ozen I, Demirkol M, Gartner KH, et al. Pharmacokinetics of orally administered tetrahydrobiopterin in patients with phenylalanine hydroxylase deficiency. J Inherit Metab Dis. 2006;29(6):725-31.

454. Leuret O, Barth M, Kuster A, Eyer D, de Parscau L, Odent S, et al. Efficacy and safety of $\mathrm{BH} 4$ before the age of 4 years in patients with mild phenylketonuria. J Inherit Metab Dis. 2012;35(6):975-81.

455. Longo N, Arnold GL, Pridjian G, Enns GM, Ficicioglu C, Parker S, et al. Longterm safety and efficacy of sapropterin: the PKUDOS registry experience. Mol Genet Metab. 2015;114(4):557-63.

456. Longo N, Siriwardena K, Feigenbaum A, Dimmock D, Burton BK, Stockler S, et al. Long-term developmental progression in infants and young children taking sapropterin for phenylketonuria: a two-year analysis of safety and efficacy. Genet Med. 2015;17(5):365-73.

457. Shintaku H, Ohura T. Sapropterin is safe and effective in patients less than 4-years-old with BH4-responsive phenylalanine hydrolase deficiency. J Pediatr. 2014;165(6):1241-4

458. Somaraju UR, Merrin M. Sapropterin dihydrochloride for phenylketonuria Cochrane Database Syst Rev. 2015;3:CD008005

459. Trefz FK, Burton BK, Longo N, Casanova MM, Gruskin DJ, Dorenbaum A, et al. Efficacy of sapropterin dihydrochloride in increasing phenylalanine tolerance in children with phenylketonuria: a phase III, randomized, doubleblind, placebo-controlled study. J Pediatr. 2009;154(5):700-7.

460. Burton BK, Bausell H, Katz R, Laduca H, Sullivan C. Sapropterin therapy increases stability of blood phenylalanine levels in patients with $\mathrm{BH} 4$ responsive phenylketonuria (PKU). Mol Genet Metab. 2010;101(2-3):110-4.

461. Kure S, Hou DC, Ohura T, Iwamoto H, Suzuki S, Sugiyama N, et al. Tetrahydrobiopterin-responsive phenylalanine hydroxylase deficiency. J Pediatr. 1999;135(3):375-8.

462. Trefz FK, Aulela-Scholz C, Blau N. Successful treatment of phenylketonuria with tetrahydrobiopterin. Eur J Pediatr. 2001;160(5):315.

463. Trefz FK, Blau N. Potential role of tetrahydrobiopterin in the treatment of maternal phenylketonuria. Pediatrics. 2003:112(6 Pt 2):1566-9.

464. Trefz FK, Scheible D, Frauendienst-Egger G, Korall H, Blau N. Long-term treatment of patients with mild and classical phenylketonuria by tetrahydrobiopterin. Mol Genet Metab. 2005;86(Suppl 1):S75-80.

465. Trefz FK, Muntau AC, Lagler FB, Moreau F, Alm J, Burlina A, et al. The Kuvan((R)) adult maternal paediatric European registry (KAMPER) multinational observational study: baseline and 1-year data in Phenylketonuria patients responsive to Sapropterin. JIMD Rep. 2015;23:35-43.

466. Christ SE, Moffitt AJ, Peck D, White DA. The effects of tetrahydrobiopterin $(\mathrm{BH} 4)$ treatment on brain function in individuals with phenylketonuria. Neurolmage Clinical. 2013;3:539-47.

467. Feillet F, Muntau AC, Debray FG, Lotz-Havla AS, Puchwein-Schwepcke A, Fofou-Caillierez MB, et al. Use of sapropterin dihydrochloride in materna phenylketonuria. A European experience of eight cases. J Inherit Metab Dis. 2014;37(5):753-62.

468. Grange DK, Hillman RE, Burton BK, Yano S, Vockley J, Fong CT, et al. Sapropterin dihydrochloride use in pregnant women with phenylketonuria: an interim report of the PKU MOMS sub-registry. Mol Genet Metab. 2014;112(1):9-16.

469. Koch R, Moseley K, Guttler F. Tetrahydrobiopterin and maternal PKU. Mol Genet Metab. 2005;86(Suppl 1):S139-41.

470. Blau N, Belanger-Quintana A, Demirkol M, Feillet F, Giovannini M, MacDonald $\mathrm{A}$, et al. Optimizing the use of sapropterin $(\mathrm{BH}(4))$ in the management of phenylketonuria. Mol Genet Metab. 2009;96(4):158-63.

471. Bernegger C, Blau N. High frequency of tetrahydrobiopterin-responsiveness among hyperphenylalaninemias: a study of 1,919 patients observed from 1988 to 2002. Mol Genet Metab. 2002;77(4):304-13.

472. Fiege B, Bonafe L, Ballhausen D, Baumgartner M, Thony B, Meili D, et al. Extended tetrahydrobiopterin loading test in the diagnosis of cofactor-responsive phenylketonuria: a pilot study. Mol Genet Metab. 2005;86(Suppl 1):S91-5

473. Trefz F, Lichtenberger O, Blau N, Muntau AC, Feillet F, Belanger-Quintana A, et al. Tetrahydrobiopterin $(\mathrm{BH} 4)$ responsiveness in neonates with hyperphenylalaninemia: a semi-mechanistically-based, nonlinear mixedeffect modeling. Mol Genet Metab. 2015:114(4):564-9.

474. Cerone R, Andria G, Giovannini M, Leuzzi V, Riva E, Burlina A. Testing for tetrahydrobiopterin responsiveness in patients with hyperphenylalaninemia due to phenylalanine hydroxylase deficiency. Adv Ther. 2013;30(3):212-28.

475. Longo N, Harding CO, Burton BK, Grange DK, Vockley J, Wasserstein M, et al. Single-dose, subcutaneous recombinant phenylalanine ammonia lyase conjugated with polyethylene glycol in adult patients with phenylketonuria: an open-label, multicentre, phase 1 dose-escalation trial. Lancet. 2014;384(9937):37-44.
476. Hamman KJ, Winn SR, Harding CO. Hepatocytes from wild-type or heterozygous donors are equally effective in achieving successful therapeutic liver repopulation in murine phenylketonuria (PKU). Molecular genetics and metabolism. 2011;104(3):235-40.

477. Viecelli HM, Harbottle RP, Wong SP, Schlegel A, Chuah MK, VandenDriessche $T$, et al. Treatment of phenylketonuria using minicircle-based naked-DNA gene transfer to murine liver. Hepatology. 2014;60(3):1035-43.

478. Awiszus D, Unger I. Coping with PKU: results of narrative interviews with parents. Eur J Pediatr. 1990;149(Suppl 1):S45-51.

479. Zwiesele S, Bannick A, Trepanier A. Parental strategies to help children with phenylketonuria (PKU) cope with feeling different. American journal of medical genetics Part A. 2015;167A(8):1787-95

480. Di Ciommo V, Forcella E, Cotugno G. Living with phenylketonuria from the point of view of children, adolescents, and young adults: a qualitative study. Journal of developmental and behavioral pediatrics : JDBP. 2012;33(3):229-35.

481. Vegni E, Fiori L, Riva E, Giovannini M, Moja EA. How individuals with phenylketonuria experience their illness: an age-related qualitative study. Child Care Health Dev. 2010;36(4):539-48.

482. Bilginsoy C, Waitzman N, Leonard CO, Ernst SL. Living with phenylketonuria: perspectives of patients and their families. J Inherit Metab Dis. 2005;28(5): 639-49.

483. Bernstein LE, Helm JR, Rocha JC, Almeida MF, Feillet F, Link RM, et al. Nutrition education tools used in phenylketonuria: clinician, parent and patient perspectives from three international surveys. J Hum Nutr Diet. 2014;27(Suppl 2):4-11.

484. Gross PA, Greenfield S, Cretin S, Ferguson J, Grimshaw J, Grol R, et al. Optimal methods for guideline implementation: conclusions from Leeds Castle meeting. Med Care. 2001;39(8 Suppl 2):II85-92.

485. Sociedade Portuguesa de Doenças Metabólicas. 2007.

486. Recommendations on the dietary management of phenylketonuria. Report of Medical Research Council Working Party on Phenylketonuria. Archives of disease in childhood. 1993;68(3):426-427

487. French Guidelines. Phenylketonuria: National Diagnosis and Treatment Protocol. 2010.

488. Rasmussen KM, Catalano PM, Yaktine AL. New guidelines for weight gain during pregnancy: what obstetrician/gynecologists should know. Current opinion in obstetrics \& gynecology. 2009;21(6):521-6.

489. Stamm RA, Houghton LA. Nutrient intake values for folate during pregnancy and lactation vary widely around the world. Nutrients. 2013:5(10):3920-47.

\section{Submit your next manuscript to BioMed Central and we will help you at every step:}

- We accept pre-submission inquiries

- Our selector tool helps you to find the most relevant journal

- We provide round the clock customer support

- Convenient online submission

- Thorough peer review

- Inclusion in PubMed and all major indexing services

- Maximum visibility for your research

Submit your manuscript at www.biomedcentral.com/submit
) Biomed Central 